\title{
Asymmetric Hydroarylation of Enones via Nickel-Catalyzed 5-endo-trig Cyclization
}

\author{
Xurong Qin, ${ }^{\ddagger}$ Marcus Wen Yao Lee, ${ }^{\ddagger}$ and Jianrong Steve Zhou*† \\ $\dagger$ State Key Laboratory of Chemical Oncogenomics, Key Laboratory of Chemical Genomics, School of \\ Chemical Biology and Biotech-nology, Peking University Shenzhen Graduate School, Nanshan District, \\ Shenzhen 518055, China. \\ \$Division of Chemistry and Bio-logical Chemistry, School of Physical and Mathematical Sciences, Nanyang \\ Technological University, 21 Nanyang Link, Singapore 637371
}

Experimental procedures and compound characterization

\section{Table of Contents}

I. General information

II. Reaction optimization

III. Asymmetric reductive cyclization

IV. One-pot reductive cyclization, aldol condensation and dimerization

V. Asymmetric synthesis towards resvertrol-based oligomers

VI. Asymmetric synthesis towards $(R)$-Rolterodine

VII. Asymmetric synthesis of deuteriated indanones

VIII. Asymmetric synthesis of (+)-Indatraline, $(+)$-Indatraline-1- $d_{1}$ and (+)-Indatraline-2,2- $d_{2}$

IX. Asymmetric synthesis of (+)-Multisianthol

X. Comparison between nickel-catalyzed reductive cyclization and palladium-catalyzed reductive Heck reactions

XI. Synthesis of $(E)-2^{\prime}$-bromochalcones 
XII. Reference

\section{General information}

All NMR spectra were acquired on Bruker $500 \mathrm{MHz}, 400 \mathrm{MHz}$ or $300 \mathrm{MHz}$ NMR spectrometers. ${ }^{1} \mathrm{H}$ NMR chemical shifts were recorded relative to TMS $(\delta 0.00)$ or residual protiated solvents $\left(\mathrm{CDCl}_{3:} \delta 7.26\right)$. Multiplicities were given as: s (singlet), d (doublet), $\mathrm{t}$ (triplet), q (quartet) and $\mathrm{m}$ (multiplet). The number of protons (n) for a given resonance was indicated by $\mathrm{nH}$. Coupling constants were reported as a $J$ value in $\mathrm{Hz}$. ${ }^{13} \mathrm{C}$ NMR spectra were obtained at $125 \mathrm{MHz}$ on $500 \mathrm{MHz}, 100 \mathrm{MHz}$ on $400 \mathrm{MHz}$ or $75 \mathrm{MHz}$ on $300 \mathrm{MHz} \mathrm{NMR}$ instruments and chemical shifts were recorded relative to solvent resonance $\left(\mathrm{CDCl}_{3}: \delta 77.16\right) .{ }^{19} \mathrm{~F}$ NMR spectra were recorded at $376 \mathrm{MHz}$ on $400 \mathrm{MHz}$ NMR spectrometers without any external standard. Proof of purity of new compounds was demonstrated with copies of ${ }^{1} \mathrm{H},{ }^{13} \mathrm{C}$, and ${ }^{19} \mathrm{~F}$ NMR spectra.

Glassware was dried at $120^{\circ} \mathrm{C}$ for at least 3 hours before use. THF and 1,4-dioxane were distilled from sodium under argon and stored over activated $4 \AA$ molecular sieve beads in an argon-filled glove box. DMA, DMF (Sigma-Aldrich), NMP, DMPU, DMSO, MeCN, $i$-PrOH and EtOH were stored over activated $4 \AA$ molecular sieve beads in an argon-filled glove box. Mn powder ( -325 mesh, $\geq 99 \%$ trace metals basis) was ordered from Sigma-Aldrich. Unless noted otherwise, commercially available chemicals were used as received without purification. The GC internal standard $n-\mathrm{C}_{12} \mathrm{H}_{26}$ was degassed with argon and dried over activated $4 \AA$ molecular sieve beads before use.

Flash chromatography was performed using Merck 40-63D60§̊ silica gel. Gas chromatography (GC) analysis was performed on a Shimadzu GC-2010 instrument with Agilent J \& W GC column DB-5MS-UI. GC/MS analysis was conducted on a Thermo Scientific DSQ II single quadrupole GC/MS instrument with Agilent J \& W GC column DB-5MS-UI. Chiral HPLC analysis was performed on a Shimadzu LC-20AD instrument using Daicel Chiralcel columns at $25^{\circ} \mathrm{C}$ and a mixture of HPLC-grade hexanes and isopropanol as eluent. Optical rotation was measured using a JASCO P-1030 polarimeter equipped with a sodium vapor lamp at $589 \mathrm{~nm}$ and the concentration of samples was denoted as $c$. GC/MS analysis was conducted on a Thermo Scientific DSQ II single quadrupole GC/MS instrument with Agilent J \& W GC column DB-5MS-UI. ESI/MS analysis was conducted on a Thermo Finnigan LCQ Fleet MS spectrometer. High resolution mass spectral analysis (HRMS) was performed on Finnigan MAT 95 XP mass spectrometer (Thermo Electron Corporation).

\section{Reaction optimization}

A typical procedure for condition optimization: In an argon-filled glove box, $\mathrm{NiBr}_{2}(\mathrm{dme})(1.5 \mathrm{mg}, 0.005 \mathrm{mmol}$, $5 \mathrm{~mol} \%$ ), ligand L7 (2.0 mg, $0.006 \mathrm{mmol}, 6 \mathrm{~mol} \%$ ) and anhydrous DMF (0.5 mL) were charged into a dry 10mL Schlenk tube (Note: we cannot ascertain the content of trace amounts of water in DMF). After stirring for about $10 \mathrm{~min}$ at room temperature, $\mathrm{Li}_{2} \mathrm{CO}_{3}(0.3 \mathrm{mmol}, 22 \mathrm{mg}, 3$ equiv), $\mathrm{Mn}$ powder $(0.3 \mathrm{mmol}, 16.5 \mathrm{mg}, 3$ 
equiv), $\mathrm{H}_{2} \mathrm{O}(0.1 \mathrm{mmol}, 1.8 \mu \mathrm{L}, 1$ equiv), GC standard $n$-dodecane $(10 \mu \mathrm{L})$, dry THF $(0.5 \mathrm{~mL})$ and $(E)$ - $o$ bromochalcone $(0.1 \mathrm{mmol}, 29 \mathrm{mg})$ were added sequentially. The reaction mixture was capped tightly and vigorously stirred in a pre-warmed oil bath maintained at $80^{\circ} \mathrm{C}$ for 24 hours. The reaction mixture was cooled to room temperature and an aliquot of the reaction mixture was passed through a short plug of silica gel with diethyl ether washings. The filtrate was subjected to GC to determine the conversion of $(E)$-o-bromochalcone and the calibrated yield of the cyclization product. Chiral HPLC analysis (OJ-H, $n$-hexane/isopropanol 95/5, flow rate $=1.0 \mathrm{~mL} / \mathrm{min}$.) was performed on the filtrate to determine the enantioselectivity of the product.

Table S1. Effect of chiral ligands in the cyclization of aryl bromide 1a<smiles>O=C(/C=C/c1ccccc1)c1ccccc1Br</smiles>

$1 a$

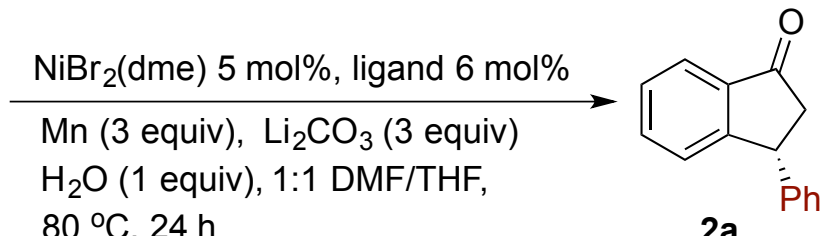

$80^{\circ} \mathrm{C}, 24 \mathrm{~h}$

$2 a$

Entry $\quad$ Conv (\%) Yield 2a (\%)


7<smiles>CC(C)(C)C1=N/C(=C(/C#N)C2=N[C@H](C(C)(C)C)CO2)OC1</smiles>

100

0

8

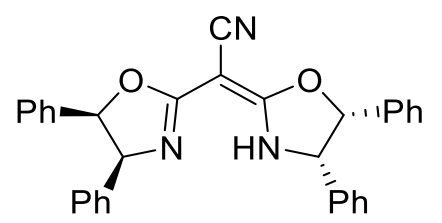

100

62

87

9<smiles>N#CC(C1=NC2Cc3ccccc3C2C1)=C1NC2Cc3ccccc3C2O1</smiles>

100

0

10<smiles>N#C/C(C1=NC(c2ccccc2)CO1)=C1\NC(c2ccccc2)CO1</smiles>

100

91

88

11

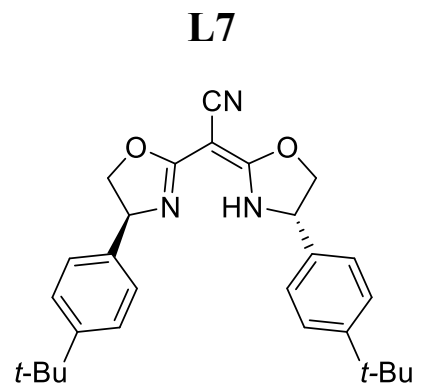

100

86

91

\section{L8}

12

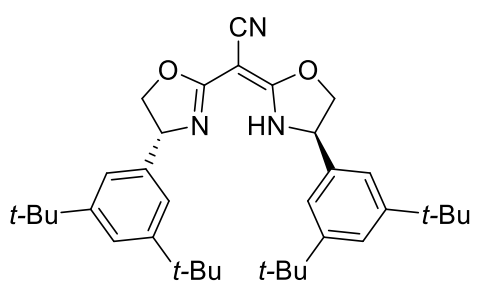

100

87

$-90$

13<smiles>c1ccc(P2COC(c3ccccc3P(c3ccccc3)c3ccccc3)=N2)cc1</smiles>

100

0

14

(R)-BINAP

100

0

15

(R)-Segphos

100

0

16

(R)-SDP

100

0 


\begin{tabular}{lcccc}
17 & $(R, R)-$ QuinoxP* & 100 & 0 & - \\
18 & $(S, S)$-Ph-BPE & 100 & 0 & - \\
19 & $(S, S)$ - Chiraphos & 100 & 0 & - \\
\hline
\end{tabular}

Table S2. Effect of bases on cyclization of aryl bromide 1a

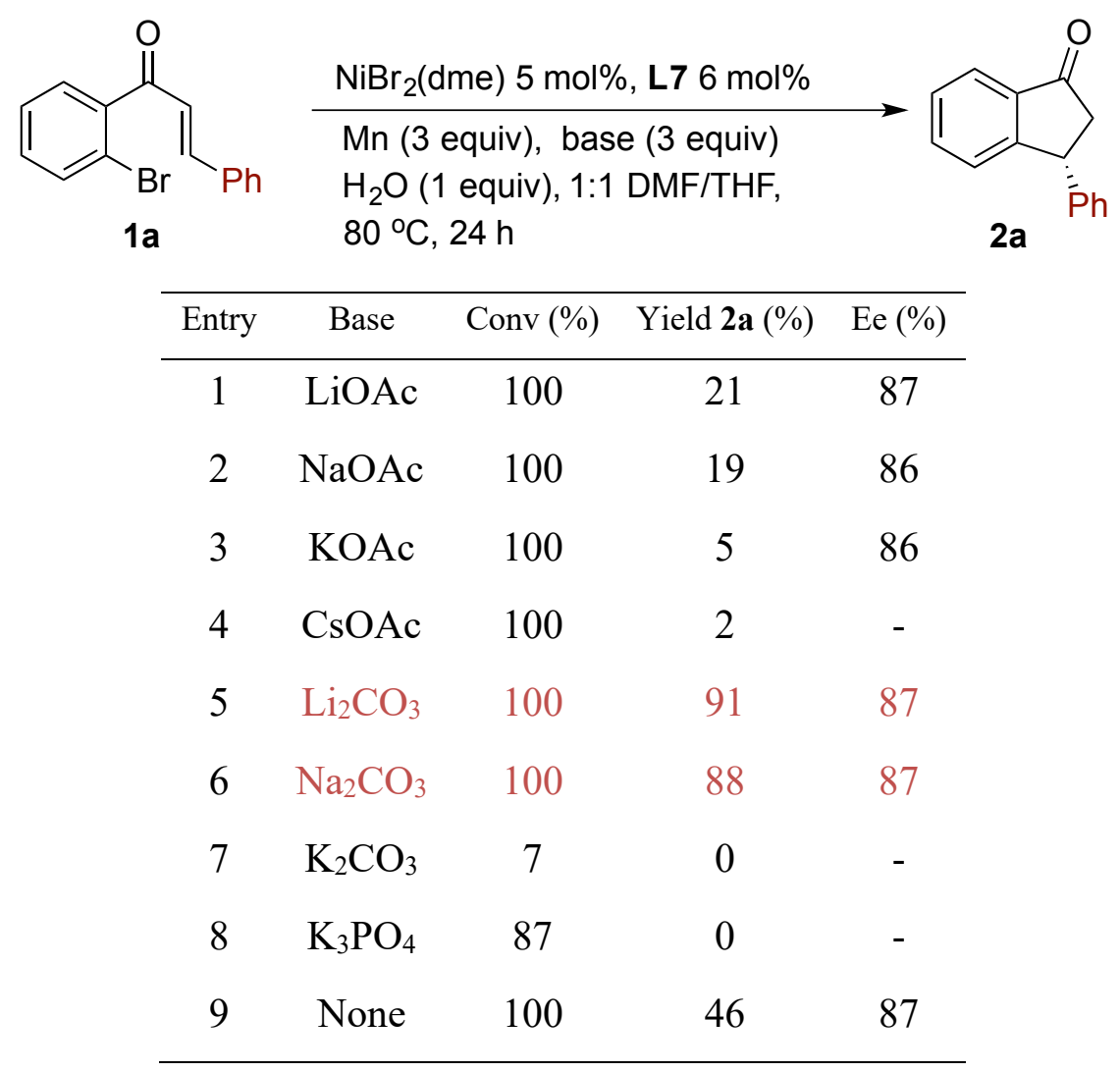

Table S3. Effect of solvents on cyclization of aryl bromide 1a

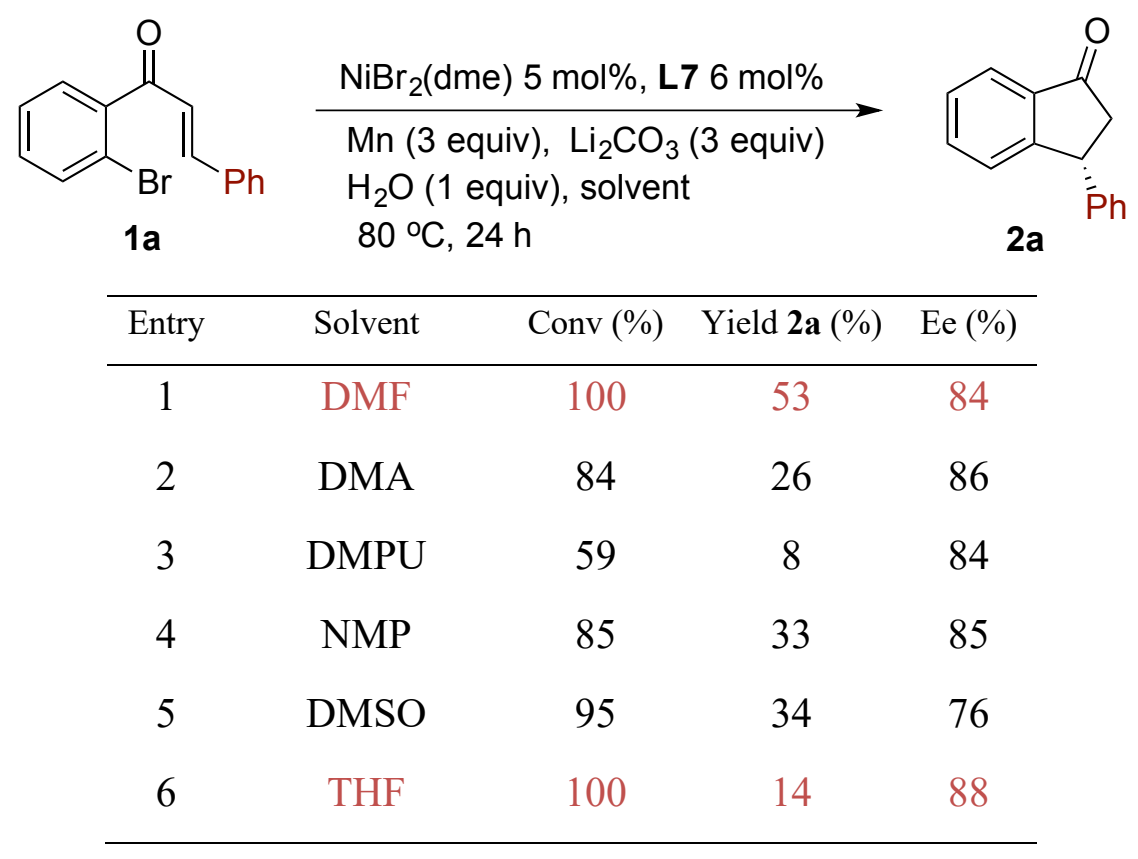




\begin{tabular}{ccccc}
\hline 7 & $1,4-$ Dioxane & 10 & 5 & 88 \\
8 & MeCN & 82 & 20 & 60 \\
9 & $i$-PrOH & 100 & 29 & 84 \\
10 & EtOH & 100 & 14 & 88 \\
11 & DMF/THF 1:1 & 100 & 91 & 87 \\
\hline
\end{tabular}

Table S4. Effect of nickel salts on the cyclization of aryl bromide 1a

\begin{tabular}{ccccc} 
Nickel salt 5 mol\%, L7 6 mol\% \\
\cline { 2 - 4 } $\begin{array}{c}\text { Mn (3 equiv), } \mathrm{Li}_{2} \mathrm{CO}_{3} \text { (3 equiv) } \\
\mathrm{H}_{2} \mathrm{O}(1 \text { equiv), } 1: 1 \text { DMF/THF, } \\
80^{\circ} \mathrm{C}, 24 \mathrm{~h}\end{array}$
\end{tabular}

Table S5. Effect of proton source on cyclization of aryl bromide 1a

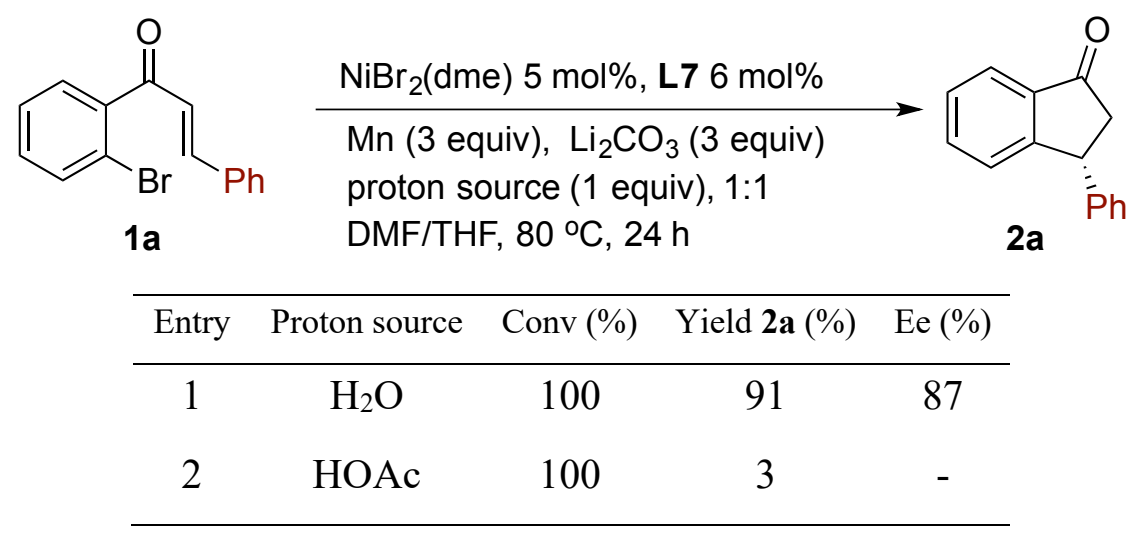


Table S6. Effect of metal powder on the cyclization of aryl bromide 1a<smiles>O=C(/C=C/c1ccccc1)c1ccccc1Br</smiles>

$1 \mathrm{a}$

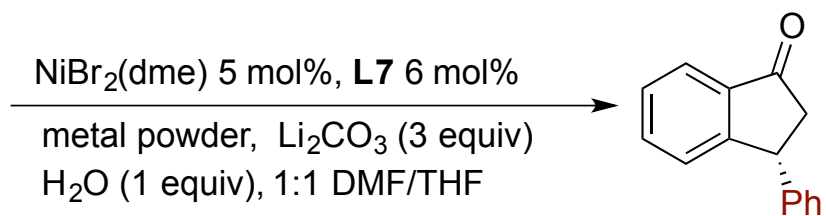

$2 a$

\begin{tabular}{ccccc}
\hline Entry & Metal powder & Conv (\%) & Yield 2a (\%) & Ee (\%) \\
\hline 1 & Mn 3 equiv & 100 & 91 & 87 \\
2 & Mn 2 equiv & 55 & 50 & 88 \\
3 & Mn 1 equiv & 9 & 7 & 88 \\
4 & Mn 0.5 equiv & 5 & 3 & 88 \\
5 & Mg 3 equiv & 5 & 2 & - \\
6 & In 3 equiv & 100 & 8 & 88 \\
7 & Zn 3 equiv & 100 & 14 & 88 \\
8 & No metal powder & 2 & 0 & - \\
\hline
\end{tabular}

\section{Asymmetric reductive cyclization}

In an argon-filled glove box, $\mathrm{NiBr}_{2}(\mathrm{dme})(3.1 \mathrm{mg}, 0.01 \mathrm{mmol}, 5 \mathrm{~mol} \%), \mathbf{L 8}$ or $\mathbf{L 9}(0.012 \mathrm{mmol}, 6 \mathrm{~mol} \%)$ and anhydrous DMF $(1.0 \mathrm{~mL})$ were charged into a dry 10-mL Schlenk tube. After stirring for about $10 \mathrm{~min}$ at room temperature, $\mathrm{Li}_{2} \mathrm{CO}_{3}$ ( $0.6 \mathrm{mmol}, 44.5 \mathrm{mg}, 3.0$ equiv), Mn powder ( $0.6 \mathrm{mmol}, 33 \mathrm{mg}, 3$ equiv), $\mathrm{H}_{2} \mathrm{O}(0.2 \mathrm{mmol}$, $3.6 \mu \mathrm{L}, 1$ equiv), GC standard $n$-dodecane $(20 \mu \mathrm{L})$, THF $(1.0 \mathrm{~mL})$ and $(E)$-o-bromochalcone $(0.2 \mathrm{mmol})$ were added sequentially. The reaction mixture was capped tightly and vigorously stirred in a pre-warmed oil bath maintained at $80-90^{\circ} \mathrm{C}$ for 24 hours. After cooled down to RT, the reaction mixture was subjected to flash chromatography with ethyl acetate/hexanes as the eluent. The enantioselectivity of the purified product was determined by chiral HPLC analysis using Daicel Chiralcel columns. Similar results can also be obtained by using standard Schlenk technique and a vacuum manifold. All of isolated compounds compared well with reported compounds in NMR characterization and optical rotation. 
<smiles>O=C1C[C@H](c2ccccc2)c2ccccc21</smiles>

\section{(R)-3-Phenyl-1-indanone (2a) [634182-03-9]}

The product was isolated by flash chromatography (ethyl acetate/hexanes 1:20) as yellow solid. $35.2 \mathrm{mg}, 85 \%$ yield. $91 \%$ ee.

$[\alpha]^{23} \mathrm{D}=-49^{\circ}\left(c=1.0, \mathrm{CHCl}_{3}\right)$. Literature value: $[\alpha]^{22} \mathrm{D}=-53.2^{\circ}\left(c=0.97, \mathrm{CHCl}_{3}\right)$ for a sample of $97 \%$ ee $(R) .{ }^{1}$ HPLC: Daicel Chiralcel OJ-H, $n$-hexane/isopropanol 95/5, flow rate $=1.0 \mathrm{~mL} / \mathrm{min}$.

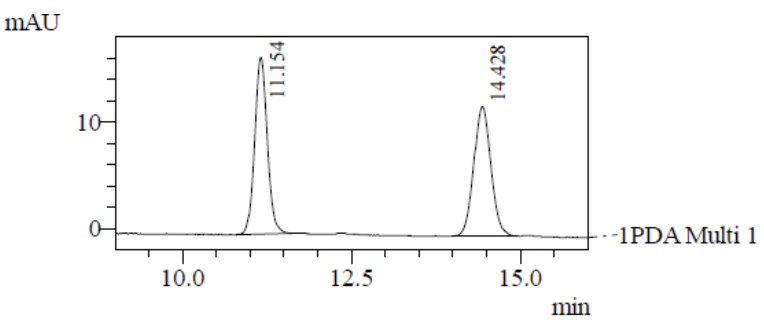

1 PDA Multi $1 / 220 \mathrm{~nm} 4 \mathrm{~nm}$

PDA Ch1 220nm 4nm
\begin{tabular}{|r|r|r|r|}
\hline Peak\# & Ret. Time & Area & Area $\%$ \\
\hline 1 & 11.154 & 219737 & 50.495 \\
\hline 2 & 14.428 & 215425 & 49.505 \\
\hline Total & & 435162 & 100.000 \\
\hline
\end{tabular}

mAU

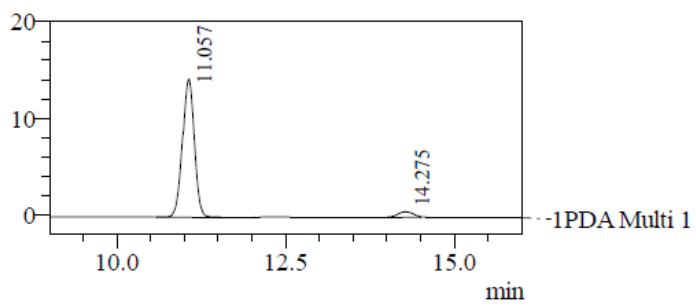

1 PDA Multi $1 / 220 \mathrm{~nm} 4 \mathrm{~nm}$

PDA Ch1 $220 \mathrm{~nm} 4 \mathrm{~nm}$
\begin{tabular}{|r|r|r|r|}
\hline \multicolumn{1}{|c|}{ Peak\# } & Ret. Time & \multicolumn{1}{c|}{ Area } & \multicolumn{1}{c|}{ Area $\%$} \\
\hline 1 & 11.057 & 175668 & 95.550 \\
\hline 2 & 14.275 & 8182 & 4.450 \\
\hline Total & & 183850 & 100.000 \\
\hline
\end{tabular}

${ }^{1} \mathrm{H}$ NMR $\left(400 \mathrm{MHz}, \mathrm{CDCl}_{3}\right): \delta 7.81(\mathrm{~d}, J=7.7 \mathrm{~Hz}, 1 \mathrm{H}), 7.57(\psi \mathrm{td}, J=7.6,1.1 \mathrm{~Hz}, 1 \mathrm{H}), 7.42(\psi \mathrm{t}, J=7.5 \mathrm{~Hz}$, 1H), 7.35-7.22 (m, 4H), 7.14-7.12 (m, 2H), $4.58(\mathrm{dd}, J=8.0,3.8 \mathrm{~Hz}, 1 \mathrm{H}), 3.23(\mathrm{dd}, J=19.2,8.1 \mathrm{~Hz}, 1 \mathrm{H}), 2.69$ (dd, $J=19.2,3.9 \mathrm{~Hz}, 1 \mathrm{H})$.

${ }^{13} \mathrm{C}$ NMR (101 $\left.\mathrm{MHz} \mathrm{CDCl}_{3}\right): \delta 206.1,158.1,143.8,136.9,135.2,129.0,128.0,127.8,127.1,127.0,123.5$, 47.0, 44.6.

MS (ESI): Calcd for $\mathrm{C}_{15} \mathrm{H}_{13} \mathrm{O}[\mathrm{M}+\mathrm{H}]^{+}:$209.10. Found: 209.15.<smiles>Cc1ccc([C@H]2CC(=O)c3ccccc32)cc1</smiles>

\section{(R)-3-(p-Tolyl)-1-indanone (2b) [2067380-63-4]}

The product was isolated by flash chromatography (ethyl acetate/hexanes 1:20) as yellow solid. $38.0 \mathrm{mg}, 86 \%$ yield. $90 \%$ ee. 
$[\alpha]^{23}{ }_{\mathrm{D}}=-62.9^{\circ}\left(c=0.66, \mathrm{CHCl}_{3}\right)$. Literature value: $[\alpha]_{\mathrm{D}}=+62.5^{\circ}\left(c=0.5, \mathrm{CHCl}_{3}\right)$ for a sample of its enantiomer in $91 \%$ ee $(S)^{2}$

HPLC: Daicel Chiralcel OJ-H, $n$-hexane/isopropanol 95/5, flow rate $=1.0 \mathrm{~mL} / \mathrm{min}$.

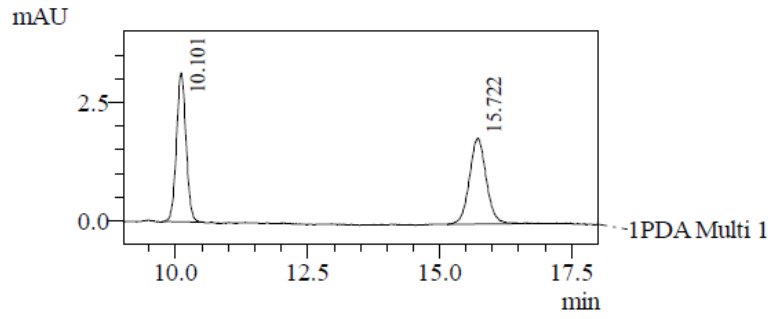

1 PDA Multi $1 / 254 \mathrm{~nm} 4 \mathrm{~nm}$

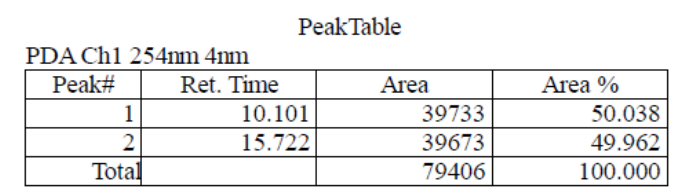

$\mathrm{mAU}$

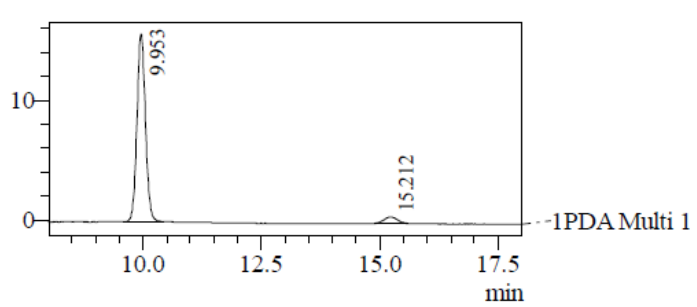

1 PDA Multi $1 / 254 \mathrm{~mm} 4 \mathrm{~nm}$

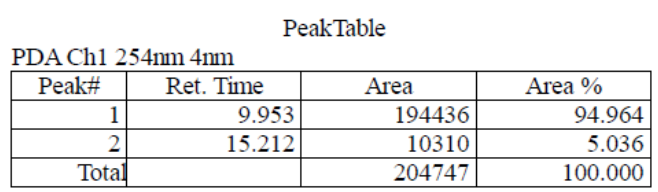

${ }^{1} \mathrm{H}$ NMR $\left(400 \mathrm{MHz}, \mathrm{CDCl}_{3}\right): \delta 7.80(\mathrm{~d}, J=7.4 \mathrm{~Hz}, 1 \mathrm{H}), 7.56(\psi \mathrm{t}, J=7.5 \mathrm{~Hz}, 1 \mathrm{H}), 7.41(\psi \mathrm{t}, J=7.4 \mathrm{~Hz}, 1 \mathrm{H})$, $7.27(\mathrm{~d}, J=7.8 \mathrm{~Hz}, 1 \mathrm{H}), 7.12$ (d, $J=7.9 \mathrm{~Hz}, 2 \mathrm{H}), 7.01$ (d, $J=8.0 \mathrm{~Hz}, 2 \mathrm{H}), 4.54(\mathrm{dd}, J=8.0,3.8 \mathrm{~Hz}, 1 \mathrm{H}), 3.22$ (dd, $J=19.2,8.0 \mathrm{~Hz}, 1 \mathrm{H}), 2.67$ (dd, $J=19.2,3.9 \mathrm{~Hz}, 1 \mathrm{H}), 2.33$ (s, 3H).

${ }^{13} \mathrm{C} \mathrm{NMR}\left(101 \mathrm{MHz}, \mathrm{CDCl}_{3}\right): \delta 206.3,158.3,140.8,136.9,136.8,135.2,129.7,127.9,127.6,127.0,123.5$, 47.1, 44.2, 21.2 .

MS (ESI): Calcd for $\mathrm{C}_{16} \mathrm{H}_{15} \mathrm{O}[\mathrm{M}+\mathrm{H}]^{+}:$223.11. Found: 223.14 .<smiles>CCCc1ccc(C2CC(=O)c3ccccc32)cc1</smiles>

\section{(R)-3-(4-i-propylphenyl)-1-indanone (2c)}

The product was isolated by flash chromatography (ethyl acetate/hexanes 1:25) as yellow oil. $40.5 \mathrm{mg}, 81 \%$ yield. $94 \%$ ee.

$[\alpha]^{23} \mathrm{D}=-54.9^{\circ}\left(c=0.79, \mathrm{CHCl}_{3}\right)$.

HPLC: Daicel Chiralcel OJ-H, $n$-hexane/isopropanol 95/5, flow rate $=1.0 \mathrm{~mL} / \mathrm{min}$. 


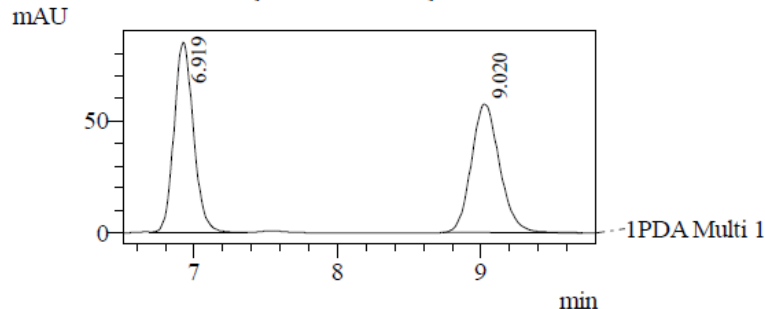

1 PDA Multi $1 / 220 \mathrm{~nm} 4 \mathrm{~nm}$

PDA Ch1 $220 \mathrm{~nm}$ 4nm
\begin{tabular}{|r|r|r|r|}
\hline Peak\# & Ret. Time & Area & \multicolumn{1}{c|}{ Area $\%$} \\
\hline 1 & 6.919 & 793465 & 50.304 \\
\hline 2 & 9.020 & 783875 & 49.696 \\
\hline Total & & 1577340 & 100.000 \\
\hline
\end{tabular}

$\mathrm{mAU}$

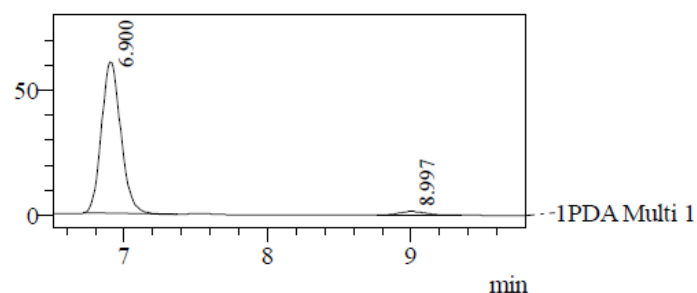

1 PDA Multi $1 / 220 \mathrm{~nm} 4 \mathrm{~nm}$

PDA Ch1 $220 \mathrm{~nm} 4 \mathrm{~nm}$
\begin{tabular}{|r|r|r|r|}
\hline \multicolumn{1}{|c|}{ Peak\# } & Ret. Time & \multicolumn{1}{c|}{ Area } & \multicolumn{1}{c|}{ Area $\%$} \\
\hline 1 & 6.900 & 568186 & 96.808 \\
\hline 2 & 8.997 & 18735 & 3.192 \\
\hline Total & & 586921 & 100.000 \\
\hline
\end{tabular}

${ }^{1} \mathrm{H}$ NMR $\left(400 \mathrm{MHz}, \mathrm{CDCl}_{3}\right): \delta 7.80(\mathrm{~d}, J=7.7 \mathrm{~Hz}, 1 \mathrm{H}), 7.56(\psi \mathrm{td}, J=7.6,1.2 \mathrm{~Hz}, 1 \mathrm{H}), 7.40(\psi \mathrm{t}, J=7.4 \mathrm{~Hz}$, 1H), $7.29(\mathrm{~d}, J=7.7 \mathrm{~Hz}, 1 \mathrm{H}), 7.17(\mathrm{~d}, J=8.1 \mathrm{~Hz}, 2 \mathrm{H}), 7.04(\mathrm{~d}, J=8.1 \mathrm{~Hz}, 2 \mathrm{H}), 4.55(\mathrm{dd}, J=7.9,3.8 \mathrm{~Hz}, 1 \mathrm{H})$, $3.21(\mathrm{dd}, J=19.2,8.0 \mathrm{~Hz}, 1 \mathrm{H}), 2.95-2.83(\mathrm{~m}, 1 \mathrm{H}), 2.69(\mathrm{dd}, J=19.2,3.9 \mathrm{~Hz}, 1 \mathrm{H}), 1.24(\mathrm{dd}, J=6.9,0.7 \mathrm{~Hz}$, $6 \mathrm{H})$.

${ }^{13} \mathrm{C} \mathrm{NMR}\left(101 \mathrm{MHz}, \mathrm{CDCl}_{3}\right): \delta 206.3,158.3,147.7,141.1,136.9,135.1,127.9,127.7,127.06,127.05,123.5$, 47.0, 44.2, 33.9, 24.1 .

HRMS (ESI ${ }^{+}$): Calcd for $\mathrm{C}_{18} \mathrm{H}_{19} \mathrm{O}[\mathrm{M}+\mathrm{H}]^{+}:$251.1430. Found: 251.1433.<smiles>CC(C)(C)c1ccc(C2CC(=O)c3ccccc32)cc1</smiles>

\section{(R)-3-[4-(t-Butyl)phenyl]-1-indanone (2d)}

The product was isolated by flash chromatography (ethyl acetate/hexanes 1:25) as yellow oil. $39.5 \mathrm{mg}, 75 \%$ yield. $93 \%$ ee.

$[\alpha]^{23} \mathrm{D}=-47.6^{\circ}\left(c=0.89, \mathrm{CHCl}_{3}\right)$. Literature value: $[\alpha]_{\mathrm{D}}=+54.2^{\circ}\left(c=0.5, \mathrm{CHCl}_{3}\right)$ for a sample of its enantiomer in $89 \%$ ee $(S){ }^{3}$

HPLC: Daicel Chiralcel OJ-H, $n$-hexane/isopropanol 95/5, flow rate $=1.0 \mathrm{~mL} / \mathrm{min}$. 
mAU

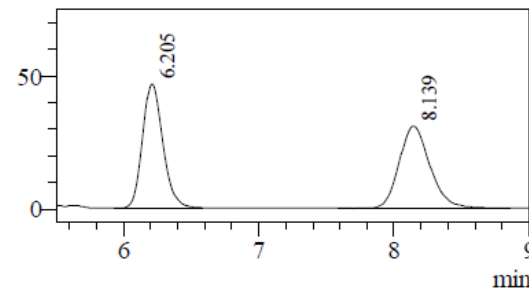

1 PDA Multi $1 / 220 \mathrm{~nm} 4 \mathrm{~nm}$

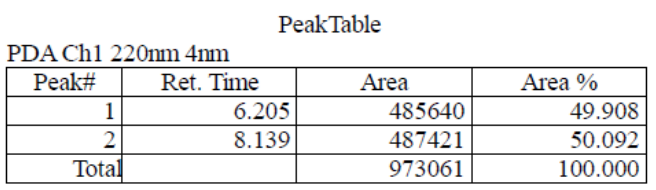

$\mathrm{mAU}$

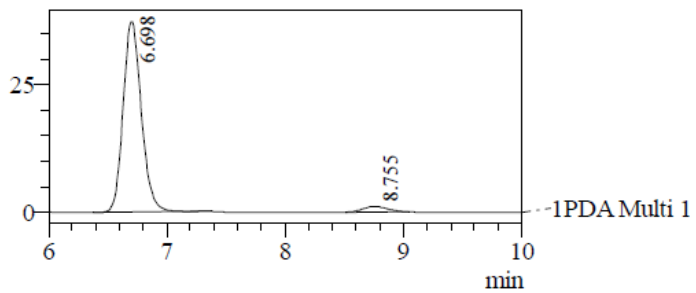

1 PDA Multi $1 / 220 \mathrm{~nm} 4 \mathrm{~nm}$

PeakTable

PDA Ch $1220 \mathrm{~nm} 4 \mathrm{~nm}$
\begin{tabular}{|r|r|r|r|}
\hline Peak\# & Ret. Time & \multicolumn{1}{c|}{ Area } & Area $\%$ \\
\hline 1 & 6.698 & 397383 & 96.192 \\
\hline 2 & 8.755 & 15730 & 3.808 \\
\hline Total & & 413113 & 100.000 \\
\hline
\end{tabular}

${ }^{1} \mathrm{H}$ NMR (400 MHz, $\left.\mathrm{CDCl}_{3}\right): \delta 7.81(\mathrm{~d}, J=7.7 \mathrm{~Hz}, 1 \mathrm{H}), 7.56(\psi \mathrm{t}, J=7.5 \mathrm{~Hz}, 1 \mathrm{H}), 7.41(\psi \mathrm{t}, J=7.4 \mathrm{~Hz}, 1 \mathrm{H})$, 7.33-7.26 (m, 3H), $7.05(\mathrm{~d}, J=8.3 \mathrm{~Hz}, 2 \mathrm{H}), 4.56(\mathrm{dd}, J=8.0,3.8 \mathrm{~Hz}, 1 \mathrm{H}), 3.21(\mathrm{dd}, J=19.2,8.0 \mathrm{~Hz}, 1 \mathrm{H}), 2.70$ (dd, $J=19.2,3.8 \mathrm{~Hz}, 1 \mathrm{H}), 1.31(\mathrm{~s}, 9 \mathrm{H})$.

${ }^{13} \mathrm{C} \mathrm{NMR}\left(101 \mathrm{MHz}, \mathrm{CDCl}_{3}\right): \delta 206.3,158.3,150.0,140.7,136.9,135.1,127.9,127.4,127.1,125.9,123.5$, 47.0, 44.1, 34.6, 31.5.

HRMS (ESI ${ }^{+}$: Calcd for $\mathrm{C}_{19} \mathrm{H}_{21} \mathrm{O}[\mathrm{M}+\mathrm{H}]^{+}: 265.1587$. Found: 265.1591 .<smiles>COc1ccc(C2CC(=O)c3ccccc32)cc1</smiles>

\section{(R)-3-(4-Methoxyphenyl)-1-indanone (2e) [1638888-93-3]}

The product was isolated by flash chromatography (ethyl acetate/hexanes 1:15) as yellow solid. $34.8 \mathrm{mg}, 73 \%$ yield. $89 \%$ ee.

$[\alpha]^{23} \mathrm{D}=-45.7^{\circ}\left(c=0.78, \mathrm{CHCl}_{3}\right)$. Literature value: $[\alpha]^{23} \mathrm{D}=-52^{\circ}\left(c=1, \mathrm{CHCl}_{3}\right)$ for a sample of $97 \%$ ee $(R){ }^{4}$ HPLC: Daicel Chiralcel OJ-H, $n$-hexane/isopropanol 95/5, flow rate $=1.0 \mathrm{~mL} / \mathrm{min}$.

mAU

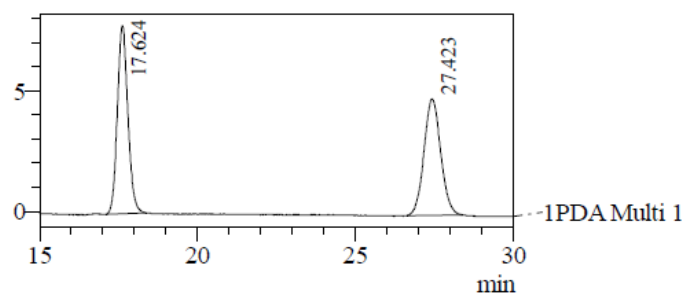

1 PDA Multi $1 / 220 \mathrm{~nm} 4 \mathrm{~nm}$

PDA Ch1 $220 \mathrm{~nm} 4 \mathrm{~nm}$
\begin{tabular}{|r|r|r|r|}
\hline Peak\# & Ret. Time & Area & Area $\%$ \\
\hline 1 & 17.624 & 180581 & 50.419 \\
\hline 2 & 27.423 & 177582 & 49.581 \\
\hline Total & & 358163 & 100.000 \\
\hline
\end{tabular}

$\mathrm{mAU}$

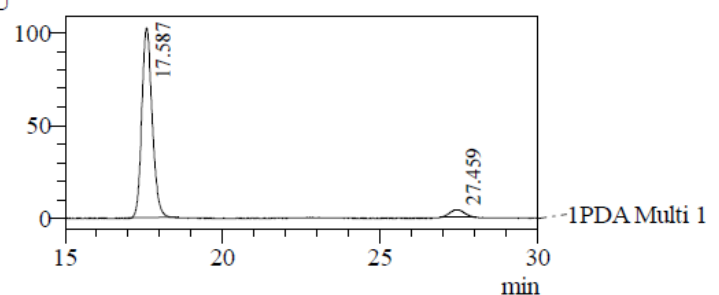

1 PDA Multi $1 / 200 \mathrm{~nm} 4 \mathrm{~nm}$

PDA Ch1 $200 \mathrm{~nm}$ 4nm
\begin{tabular}{|r|r|r|r|}
\hline Peak\# & Ret. Time & \multicolumn{1}{|c|}{ Area } & Area $\%$ \\
\hline 1 & 17.587 & 2345648 & 94.547 \\
\hline 2 & 27.459 & 135291 & 5.453 \\
\hline Total & & 2480939 & 100.000 \\
\hline
\end{tabular}


${ }^{1} \mathrm{H}$ NMR (400 MHz, $\left.\mathrm{CDCl}_{3}\right): \delta 7.80(\mathrm{~d}, J=7.7 \mathrm{~Hz}, 1 \mathrm{H}), 7.57(\psi \mathrm{td}, J=7.6,1.2 \mathrm{~Hz}, 1 \mathrm{H}), 7.41(\psi \mathrm{t}, J=7.4 \mathrm{~Hz}$, $1 \mathrm{H}), 7.29-7.24(\mathrm{~m}, 1 \mathrm{H}), 7.07-7.00(\mathrm{~m}, 2 \mathrm{H}), 6.88-6.82(\mathrm{~m}, 2 \mathrm{H}), 4.53(\mathrm{dd}, J=8.0,3.8 \mathrm{~Hz}, 1 \mathrm{H}), 3.79(\mathrm{~s}, 3 \mathrm{H}), 3.22$ (dd, $J=19.2,8.0 \mathrm{~Hz}, 1 \mathrm{H}), 2.65(\mathrm{dd}, J=19.2,3.9 \mathrm{~Hz}, 1 \mathrm{H})$.

${ }^{13} \mathrm{C} \mathrm{NMR}\left(101 \mathrm{MHz}, \mathrm{CDCl}_{3}\right): \delta 206.3,158.7,158.4,136.9,135.9,135.2,128.8,127.9,127.0,123.5,114.4$, $55.4,47.2,43.8$.

MS (ESI): Calcd for $\mathrm{C}_{16} \mathrm{H}_{15} \mathrm{O}_{2}[\mathrm{M}+\mathrm{H}]^{+}:$239.11. Found: 239.04 .<smiles>CN(C)c1ccc(C2CC(=O)c3ccccc32)cc1</smiles>

\section{(R)-3-(4-Dimethylaminophenyl)-1-indanone (2f) [1713297-97-2]}

The product was isolated by flash chromatography (ethyl acetate/hexanes 1:15) as pink solid. 29.1 mg, 58\% yield. $91 \%$ ee.

$[\alpha]^{23}{ }_{\mathrm{D}}=-36.5^{\circ}\left(c=0.56, \mathrm{CHCl}_{3}\right)$. Literature value: $[\alpha]^{23} \mathrm{D}=-83^{\circ}\left(c=1.0, \mathrm{CHCl}_{3}\right)$ for a sample of $97 \%$ ee $(R) .{ }^{4}$ HPLC: Daicel Chiralcel OD-H, $n$-hexane/isopropanol 80/20, flow rate $=0.5 \mathrm{~mL} / \mathrm{min}$.

mAU

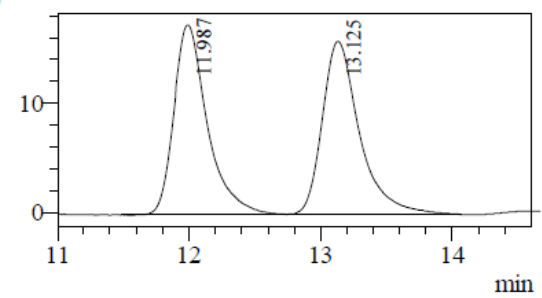

1 PDA Multi $1 / 220 \mathrm{~nm} 4 \mathrm{~nm}$

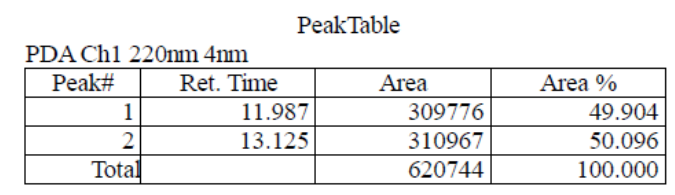

$\mathrm{mAU}$

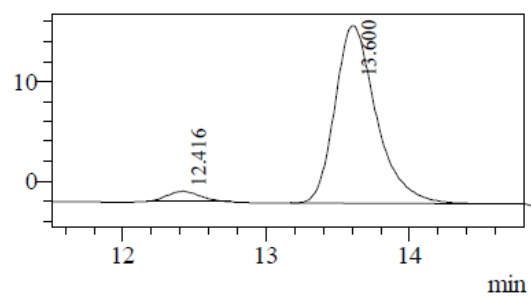

1 PDA Multi $1 / 220 \mathrm{~nm}$ 4nm

PDA Ch1 $220 \mathrm{~nm}$ 4nm
\begin{tabular}{|r|r|r|r|}
\hline Peak\# & Ret. Time & \multicolumn{1}{|c|}{ Area } & Area $\%$ \\
\hline 1 & 12.416 & 16420 & 4.384 \\
\hline 2 & 13.600 & 358159 & 95.616 \\
\hline Total & & 374579 & 100.000 \\
\hline
\end{tabular}

${ }^{1} \mathrm{H}$ NMR $\left(400 \mathrm{MHz}, \mathrm{CDCl}_{3}\right): \delta 7.79(\mathrm{~d}, J=7.7 \mathrm{~Hz}, 1 \mathrm{H}), 7.55(\psi \mathrm{td}, J=7.6,1.2 \mathrm{~Hz}, 1 \mathrm{H}), 7.39(\psi \mathrm{t}, J=7.4 \mathrm{~Hz}$, $1 \mathrm{H}), 7.30-7.24(\mathrm{~m}, 1 \mathrm{H}), 7.03-6.95(\mathrm{~m}, 2 \mathrm{H}), 6.71-6.63(\mathrm{~m}, 2 \mathrm{H}), 4.49(\mathrm{dd}, J=7.9,3.8 \mathrm{~Hz}, 1 \mathrm{H}), 3.20(\mathrm{dd}, J=19.2$, $8.0 \mathrm{~Hz}, 1 \mathrm{H}), 2.93(\mathrm{~s}, 6 \mathrm{H}), 2.66(\mathrm{dd}, J=19.2,3.9 \mathrm{~Hz}, 1 \mathrm{H})$.

${ }^{13} \mathrm{C} \mathrm{NMR}\left(101 \mathrm{MHz}, \mathrm{CDCl}_{3}\right): \delta 206.7,158.9,149.8,136.8,135.1,131.5,128.4,127.7,127.0,123.4,113.1$, 47.2, 43.8, 40.8 .

MS (ESI): Calcd for $\mathrm{C}_{17} \mathrm{H}_{18} \mathrm{NO}[\mathrm{M}+\mathrm{H}]^{+}:$252.14. Found: 252.25 . 
<smiles>O=C1C[C@H](c2ccc(F)cc2)c2ccccc21</smiles>

\section{(R)-3-(4-Fluorophenyl)-1-indanone (2g) [1422961-06-5]}

The product was isolated by flash chromatography (ethyl acetate/hexanes 1:15) as yellow solid. $40.2 \mathrm{mg}, 89 \%$ yield. $87 \%$ ee.

$[\alpha]^{23}{ }_{\mathrm{D}}=-34.9^{\circ}\left(c=1.0, \mathrm{CHCl}_{3}\right)$. Literature value: $[\alpha]^{23} \mathrm{D}=-28^{\circ}\left(c=0.50, \mathrm{CHCl}_{3}\right)$ for a sample of $92 \%$ ee $(R) .{ }^{4}$ HPLC: Daicel Chiralcel IC-3, $n$-hexane/isopropanol 95/5, flow rate $=1.0 \mathrm{~mL} / \mathrm{min}$.

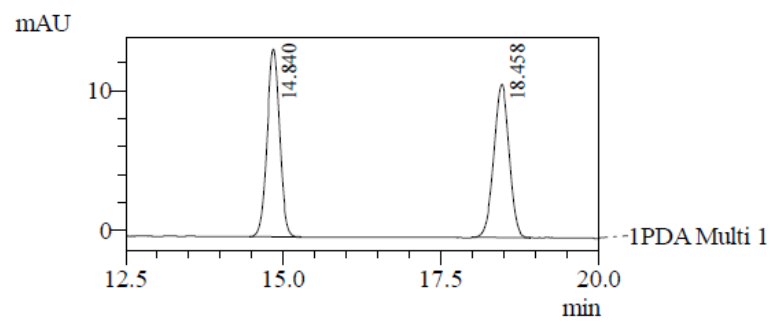

1 PDA Multi $1 / 220 \mathrm{~nm} 4 \mathrm{~nm}$

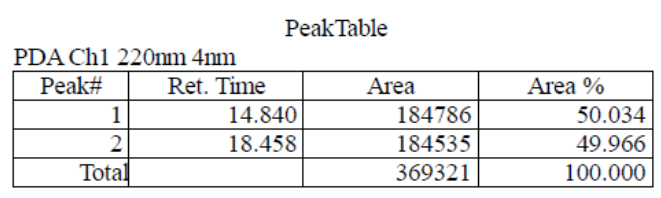

$\mathrm{mAU}$

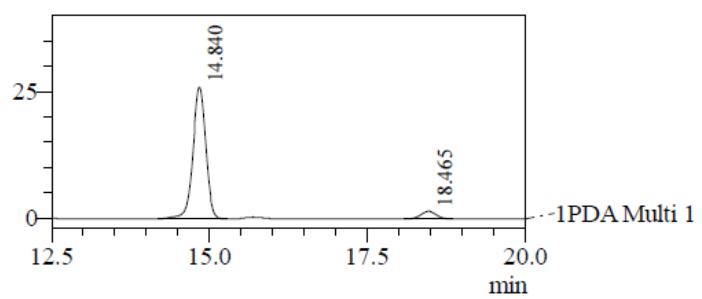

1 PDA Multi $1 / 220 \mathrm{~nm} 4 \mathrm{~nm}$

PDA Ch1 $220 \mathrm{~nm}$ 4nm
\begin{tabular}{|r|r|r|r|}
\hline Peak\# & Ret. Time & \multicolumn{1}{|c|}{ Area } & Area $\%$ \\
\hline 1 & 14.840 & 371052 & 93.488 \\
\hline 2 & 18.465 & 25845 & 6.512 \\
\hline Total & & 396898 & 100.000 \\
\hline
\end{tabular}

${ }^{1} \mathrm{H}$ NMR $\left(400 \mathrm{MHz}, \mathrm{CDCl}_{3}\right): \delta 7.82(\mathrm{~d}, J=7.7 \mathrm{~Hz}, 1 \mathrm{H}), 7.58(\psi \mathrm{td}, J=7.6,1.2 \mathrm{~Hz}, 1 \mathrm{H}), 7.46-7.40(\mathrm{~m}, 1 \mathrm{H}), 7.28$ $-7.23(\mathrm{~m}, 1 \mathrm{H}), 7.12-7.04(\mathrm{~m}, 2 \mathrm{H}), 7.03-6.97(\mathrm{~m}, 2 \mathrm{H}), 4.57(\mathrm{dd}, J=8.0,3.8 \mathrm{~Hz}, 1 \mathrm{H}), 3.23(\mathrm{dd}, J=19.2,8.1 \mathrm{~Hz}$, 1H), $2.64(\mathrm{dd}, J=19.2,3.9 \mathrm{~Hz}, 1 \mathrm{H})$.

${ }^{13} \mathrm{C} \mathrm{NMR}\left(101 \mathrm{MHz}, \mathrm{CDCl}_{3}\right): \delta 205.8,162.0\left(\mathrm{~d}, J_{C-F}=245.6 \mathrm{~Hz}\right), 157.8,139.6\left(\mathrm{~d}, J_{C-F}=3.3 \mathrm{~Hz}\right), 136.9,135.3$, $129.3\left(\mathrm{~d}, J_{C-F}=8.0 \mathrm{~Hz}\right), 128.2,126.9,123.6,115.9\left(\mathrm{~d}, J_{C-F}=21.5 \mathrm{~Hz}\right), 47.1,43.9$.

${ }^{19} \mathrm{~F}$ NMR $\left(377 \mathrm{MHz}, \mathrm{CDCl}_{3}\right): \delta-115.7$.

MS (ESI): Calcd for $\mathrm{C}_{15} \mathrm{H}_{12} \mathrm{FO}[\mathrm{M}+\mathrm{H}]^{+}:$227.09. Found: 227.29.<smiles>O=C1C[C@H](c2ccc(Cl)cc2)c2ccccc21</smiles> 
The product was isolated by flash chromatography (ethyl acetate/hexanes 1:15) as yellow solid. $31.5 \mathrm{mg}, 65 \%$ yield. $81 \%$ ee.

$[\alpha]^{23}{ }_{\mathrm{D}}=-62.4^{\circ}\left(c=0.53, \mathrm{CHCl}_{3}\right)$. Litrature value: $[\alpha]^{23} \mathrm{D}=-42^{\circ}\left(c=1.0, \mathrm{CHCl}_{3}\right)$ for a sample of $92 \%$ ee $(R){ }^{4}$ HPLC: Daicel Chiralcel OJ-H, $n$-hexane/isopropanol 95/5, flow rate $=1.0 \mathrm{~mL} / \mathrm{min}$.

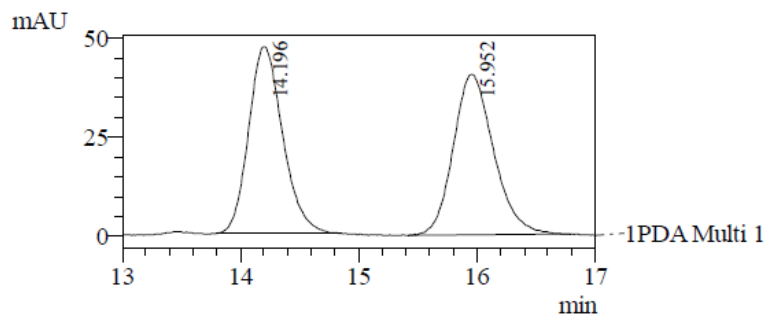

1 PDAMulti $1 / 200 \mathrm{~nm} 4 \mathrm{~nm}$

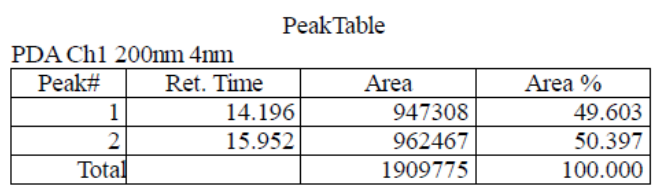

mAU

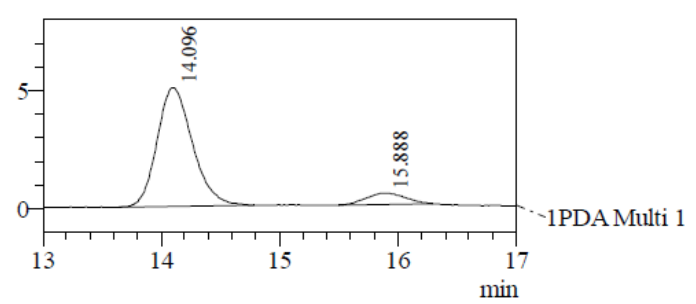

1 PDA Multi $1 / 254 \mathrm{~nm} 4 \mathrm{~nm}$

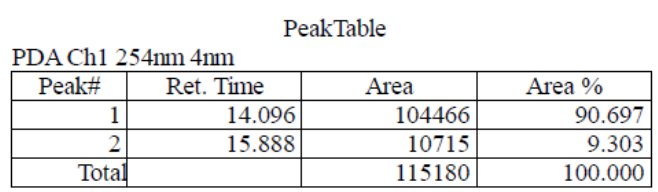

${ }^{1} \mathrm{H}$ NMR (400 MHz, $\left.\mathrm{CDCl}_{3}\right): \delta 7.82(\mathrm{~d}, J=7.7 \mathrm{~Hz}, 1 \mathrm{H}), 7.62-7.55(\mathrm{~m}, 1 \mathrm{H}), 7.43(\psi \mathrm{t}, J=7.5 \mathrm{~Hz}, 1 \mathrm{H}), 7.31-7.21$ (m, 3H), 7.09-7.04 (m, 2H), $4.56(\mathrm{dd}, J=8.0,3.8 \mathrm{~Hz}, 1 \mathrm{H}), 3.23(\mathrm{dd}, J=19.2,8.1 \mathrm{~Hz}, 1 \mathrm{H}), 2.63(\mathrm{dd}, J=19.2$, $3.9 \mathrm{~Hz}, 1 \mathrm{H})$.

${ }^{13} \mathrm{C}$ NMR $\left(101 \mathrm{MHz}, \mathrm{CDCl}_{3}\right): \delta 205.6,157.5,142.4,136.9,135.4,133.0,129.2,129.1,128.3,126.9,123.7$, 46.9, 44.0 .

MS (ESI): Calcd for $\mathrm{C}_{15} \mathrm{H}_{12} \mathrm{ClO}[\mathrm{M}+\mathrm{H}]^{+}:$243.06. Found: 243.36 .<smiles>COC(=O)c1ccc(C2CC(=O)c3ccccc32)cc1</smiles>

\section{(R)-3-(4-Methylbenzoate)-1-indanone (2i)}

The product was isolated by flash chromatography (ethyl acetate/hexanes 1:10) as yellow solid. 33.5 mg, 63\% yield. $81 \%$ ee.

$[\alpha]^{23}{ }_{\mathrm{D}}=-28.3^{\circ}\left(c=0.45, \mathrm{CHCl}_{3}\right)$.

HPLC: Daicel Chiralcel OJ-H, $n$-hexane/isopropanol 90/10, flow rate $=1.0 \mathrm{~mL} / \mathrm{min}$. 
mAU

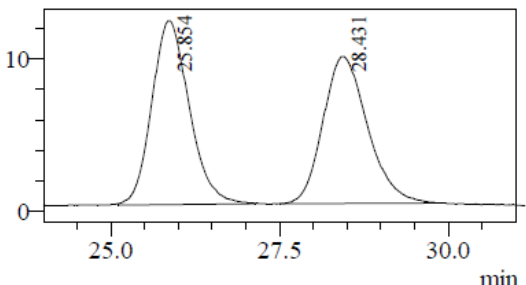

1 PDA Multi $1 / 210 \mathrm{~nm} 4 \mathrm{~nm}$

PDA Ch1 $210 \mathrm{~nm} 4 \mathrm{~nm}$
\begin{tabular}{|r|c|c|r|}
\hline Peak\# & Ret. Time & Area & Area $\%$ \\
\hline 1 & 25.854 & 462995 & 51.101 \\
\hline 2 & 28.431 & 443041 & 48.899 \\
\hline Total & & 906036 & 100.000 \\
\hline
\end{tabular}

mAU

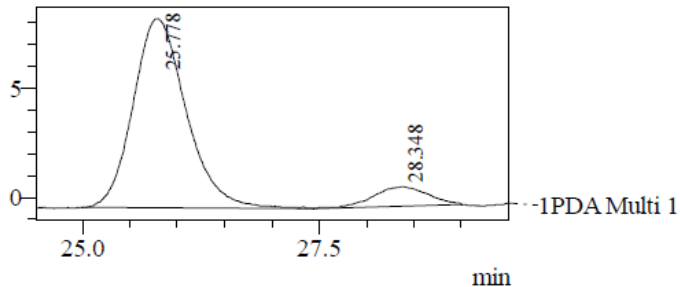

1 PDA Multi $1 / 210 \mathrm{~nm} 4 \mathrm{~nm}$

PDACh1 $210 \mathrm{~nm} 4 \mathrm{~nm}$
\begin{tabular}{|r|r|r|r|}
\hline Peak\# & Ret. Time & \multicolumn{1}{|c|}{ Area } & Area $\%$ \\
\hline 1 & 25.778 & 339398 & 90.691 \\
\hline 2 & 28.348 & 34837 & 9.309 \\
\hline Total & & 374236 & 100.000 \\
\hline
\end{tabular}

${ }^{1} \mathrm{H}$ NMR (400 MHz, $\left.\mathrm{CDCl}_{3}\right): \delta 7.99(\mathrm{~d}, J=8.4 \mathrm{~Hz}, 2 \mathrm{H}), 7.83(\mathrm{~d}, J=7.7 \mathrm{~Hz}, 1 \mathrm{H}), 7.59(\psi \mathrm{td}, J=7.6,1.1 \mathrm{~Hz}$, 1H), 7.45 ( $\psi \mathrm{t}, J=7.4 \mathrm{~Hz}, 1 \mathrm{H}), 7.24$ (d, $J=7.7 \mathrm{~Hz}, 1 \mathrm{H}), 7.20$ (d, $J=8.3 \mathrm{~Hz}, 2 \mathrm{H}), 4.64(\mathrm{dd}, J=8.0,3.8 \mathrm{~Hz}, 1 \mathrm{H})$, 3.91 (s, 3H), 3.26 (dd, $J=19.2,8.1 \mathrm{~Hz}, 1 \mathrm{H}), 2.68$ (dd, $J=19.2,3.9 \mathrm{~Hz}, 1 \mathrm{H})$.

${ }^{13} \mathrm{C} \mathrm{NMR}\left(101 \mathrm{MHz}, \mathrm{CDCl}_{3}\right): \delta 205.5,166.9,157.2,149.0,136.9,135.4,130.4,129.2,128.4,127.9,126.9$, $123.8,52.3,46.6,44.5$.

HRMS (ESI ${ }^{+}$): Calcd for $\mathrm{C}_{17} \mathrm{H}_{14} \mathrm{NaO}_{3}[\mathrm{M}+\mathrm{Na}]^{+}: 289.0835$. Found: 289.0849 .<smiles>O=C1C[C@H](c2ccc3ccccc3c2)c2ccccc21</smiles>

\section{(R)-3-(2-Naphthyl)-1-indanone (2j) [871563-53-0]}

The product was isolated by flash chromatography (ethyl acetate/hexanes 1:15) as yellow solid. $40.2 \mathrm{mg}, 78 \%$ yield. $92 \%$ ee.

$[\alpha]^{23} \mathrm{D}=-106.5^{\circ}\left(c=1.2, \mathrm{CHCl}_{3}\right)$. Literature value: $[\alpha]^{23} \mathrm{D}=-121^{\circ}\left(c=1.0, \mathrm{CHCl}_{3}\right)$ for a sample of $94 \%$ ee $(R) .{ }^{4}$ HPLC: Daicel Chiralcel IC-3, $n$-hexane/isopropanol 95/5, flow rate $=1.0 \mathrm{~mL} / \mathrm{min}$.

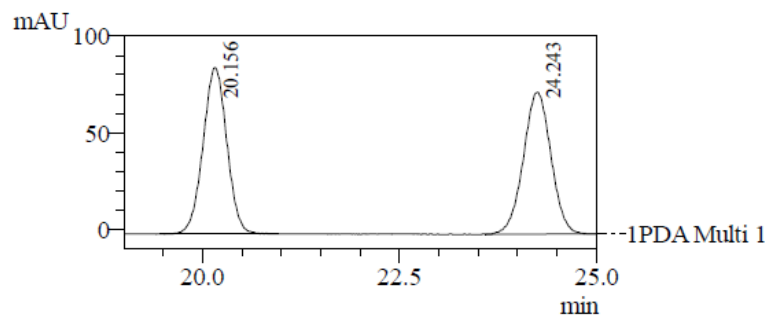

1 PDA Multi $1 / 200 \mathrm{~nm} 4 \mathrm{~nm}$

PDA Ch1 200 nm 4nm
\begin{tabular}{|r|r|r|r|}
\hline Peak\# & Ret. Time & Area & Area $\%$ \\
\hline 1 & 20.156 & 1807419 & 50.212 \\
\hline 2 & 24.243 & 1792136 & 49.788 \\
\hline Total & & 3599555 & 100.000 \\
\hline
\end{tabular}

$\mathrm{mAU}$

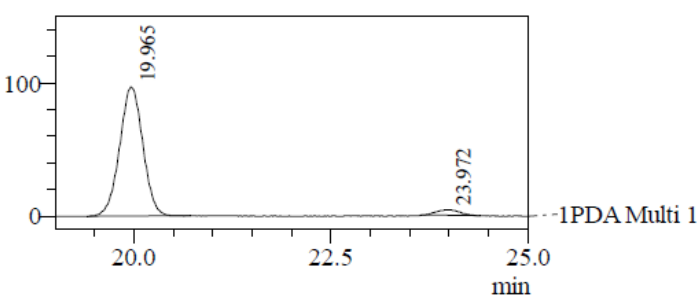

1 PDA Multi $1 / 200 \mathrm{~nm} 4 \mathrm{~nm}$

PDA Ch1 $200 \mathrm{~nm}$ 4nm
\begin{tabular}{|r|r|r|r|}
\hline Peak\# & Ret. Time & \multicolumn{1}{|c|}{ Area } & Area $\%$ \\
\hline 1 & 19.965 & 2001373 & 95.806 \\
\hline 2 & 23.972 & 87611 & 4.194 \\
\hline Total & & 2088984 & 100.000 \\
\hline
\end{tabular}


${ }^{1} \mathrm{H}$ NMR (400 MHz, $\left.\mathrm{CDCl}_{3}\right): \delta 7.85(\mathrm{~d}, J=7.7 \mathrm{~Hz}, 1 \mathrm{H}), 7.82-7.77(\mathrm{~m}, 3 \mathrm{H}), 7.66(\mathrm{~s}, 1 \mathrm{H}), 7.57(\mathrm{dd}, J=7.4,1.0$ $\mathrm{Hz}, 1 \mathrm{H}), 7.52-7.41(\mathrm{~m}, 3 \mathrm{H}), 7.29$ (d, $J=7.7 \mathrm{~Hz}, 1 \mathrm{H}), 7.14$ (dd, $J=8.5,1.6 \mathrm{~Hz}, 1 \mathrm{H}), 4.75$ (dd, $J=8.0,3.8 \mathrm{~Hz}$, 1H), $3.30(\mathrm{dd}, J=19.3,8.1 \mathrm{~Hz}, 1 \mathrm{H}), 2.78(\mathrm{dd}, J=19.3,3.9 \mathrm{~Hz}, 1 \mathrm{H})$.

${ }^{13} \mathrm{C}$ NMR (101 MHz, $\left.\mathrm{CDCl}_{3}\right): \delta 206.1,158.0,141.0,137.0,135.3,133.6,132.6,129.1,128.1,127.84,127.77$, $127.1,126.56,126.55,126.0,125.6,123.6,46.9,44.8$.

MS (ESI): Calcd for $\mathrm{C}_{19} \mathrm{H}_{15} \mathrm{O}[\mathrm{M}+\mathrm{H}]^{+}:$259.11. Found: 259.34 .

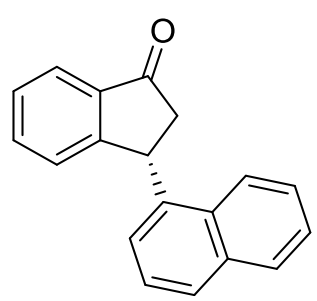

\section{(R)-3-(1-Naphthyl)-1-indanone (2k) [1713298-00-0]}

The product was isolated by flash chromatography (ethyl acetate/hexanes 1:15) as yellow solid. $44.3 \mathrm{mg}, 86 \%$ yield. $90 \%$ ee.

$[\alpha]^{23} \mathrm{D}=+193.0^{\circ}\left(c=1.1, \mathrm{CHCl}_{3}\right)$.

HPLC: Daicel Chiralcel OJ-H, $n$-hexane/isopropanol 90/10, flow rate $=0.5 \mathrm{~mL} / \mathrm{min}$.

mAU

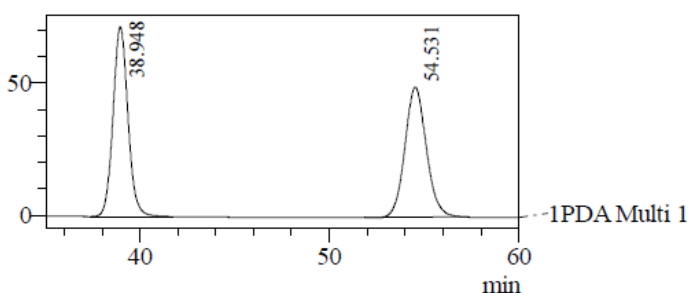

1 PDA Multi $1 / 220 \mathrm{~nm} 4 \mathrm{~nm}$

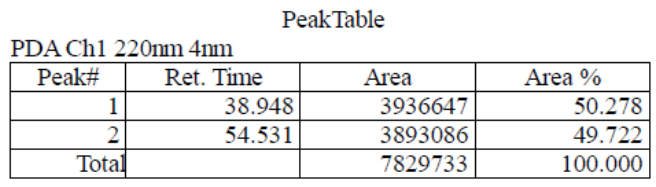

mAU

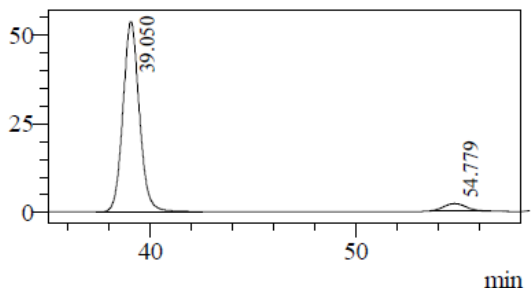

1 PDA Multi $1 / 220 \mathrm{~nm} 4 \mathrm{~nm}$

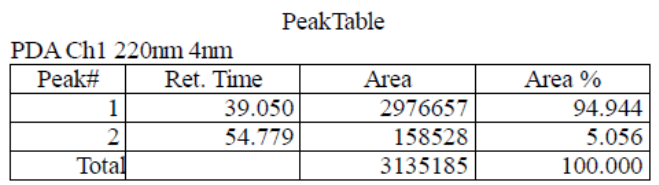

${ }^{1} \mathrm{H}$ NMR (400 MHz, $\left.\mathrm{CDCl}_{3}\right): \delta 8.23(\mathrm{~s}, 1 \mathrm{H}), 7.92-7.90(\mathrm{~m}, 2 \mathrm{H}), 7.77(\mathrm{~d}, J=8.1 \mathrm{~Hz}, 1 \mathrm{H}), 7.61-7.34(\mathrm{~m}, 6 \mathrm{H})$, $6.92(\mathrm{~s}, 1 \mathrm{H}), 5.45(\mathrm{~s}, 1 \mathrm{H}), 3.41(\mathrm{~d}, J=11.9 \mathrm{~Hz}, 1 \mathrm{H}), 2.68(\mathrm{~s}, 1 \mathrm{H})$. Broad signals are due to slow rotation around the benzyl-naphthyl single bond.

MS (ESI): Calcd for $\mathrm{C}_{19} \mathrm{H}_{15} \mathrm{O}[\mathrm{M}+\mathrm{H}]^{+}:$259.11. Found: 259.16 . 


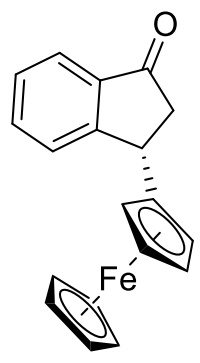

\section{(S)-3-Ferrocenyl-1-indanone (21) [1713298-08-8]}

The product was isolated by flash chromatography (ethyl acetate/hexanes 1:15) as yellow solid. $47.2 \mathrm{mg}, 75 \%$ yield. $90 \%$ ee.

$[\alpha]^{23}{ }_{\mathrm{D}}=+58.1^{\circ}\left(c=0.35, \mathrm{CHCl}_{3}\right)$. Literature value: $[\alpha]^{23}{ }_{\mathrm{D}}=+146^{\circ}\left(c=0.5, \mathrm{CHCl}_{3}\right)$ for a sample of $97 \%$ ee $(S)$. 4

HPLC: Daicel Chiralcel OJ-H, $n$-hexane/isopropanol 95/5, flow rate $=1.0 \mathrm{~mL} / \mathrm{min}$.

mAU

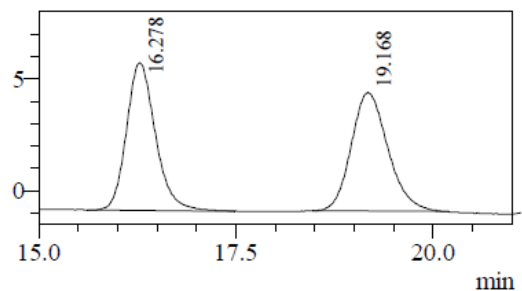

1 PDA Multi $1 / 220 \mathrm{~nm} 4 \mathrm{~nm}$

PDA Ch1 220nm 4nm
\begin{tabular}{|r|c|c|r|}
\hline \multicolumn{1}{|c|}{ Peak\# } & Ret. Time & Area & \multicolumn{1}{c|}{ Area $\%$} \\
\hline 1 & 16.278 & 167752 & 49.445 \\
\hline 2 & 19.168 & 171521 & 50.555 \\
\hline Total & & 339273 & 100.000 \\
\hline
\end{tabular}

mAU

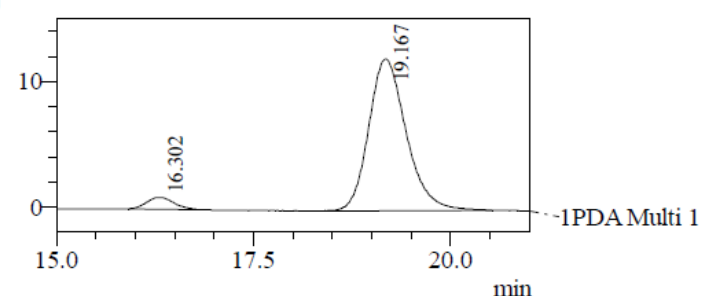

1 PDA Multi $1 / 220 \mathrm{~nm} 4 \mathrm{~nm}$

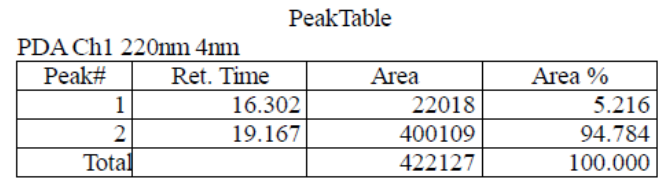

${ }^{1} \mathrm{H}$ NMR (400 MHz, $\left.\mathrm{CDCl}_{3}\right): \delta 7.74(\mathrm{~d}, J=7.6 \mathrm{~Hz}, 1 \mathrm{H}), 7.56(\psi \mathrm{t}, J=7.1 \mathrm{~Hz}, 1 \mathrm{H}), 7.45(\mathrm{~d}, J=7.6 \mathrm{~Hz}, 1 \mathrm{H})$, 7.37 ( $\psi \mathrm{t}, J=7.4 \mathrm{~Hz}, 1 \mathrm{H}), 4.31(\mathrm{dd}, J=7.7,3.4 \mathrm{~Hz}, 1 \mathrm{H}), 4.23(\mathrm{~s}, 1 \mathrm{H}), 4.18(\mathrm{~s}, 1 \mathrm{H}), 4.14(\psi \mathrm{s}, 6 \mathrm{H}), 3.97(\mathrm{~s}, 1 \mathrm{H})$, $3.20(\mathrm{dd}, J=19.0,7.9 \mathrm{~Hz}, 1 \mathrm{H}), 2.95(\mathrm{dd}, J=19.0,3.6 \mathrm{~Hz}, 1 \mathrm{H})$.

${ }^{13} \mathrm{C}$ NMR $\left(101 \mathrm{MHz}, \mathrm{CDCl}_{3}\right): \delta 206.2,158.1,136.1,134.9,127.9,126.4,123.6,91.0,68.7,68.51,68.45,67.8$, $65.5,45.4,38.3$.

MS (ESI): Calcd for $\mathrm{C}_{19} \mathrm{H}_{17} \mathrm{FeO}[\mathrm{M}+\mathrm{H}]^{+}:$317.06. Found: 317.05 .

The following examples were obtained with a nickel catalyst of ligand $\mathbf{L 9}$.<smiles>Cc1ccccc1C1CC(=O)c2ccccc21</smiles> 
The product was isolated by flash chromatography (ethyl acetate/hexanes 1:15) as yellow solid. $39.1 \mathrm{mg}, 88 \%$ yield. $95 \%$ ee.

$[\alpha]^{23}{ }_{\mathrm{D}}=-21.5^{\circ}\left(c=0.68, \mathrm{CHCl}_{3}\right)$. Literature value: $[\alpha]^{23} \mathrm{D}=+56^{\circ}\left(c=1, \mathrm{CHCl}_{3}\right)$ for a sample of its enantiomer in $98 \%$ ee $(R){ }^{4}$

HPLC: Daicel Chiralcel OJ-H, $n$-hexane/isopropanol 95/5, flow rate $=1.0 \mathrm{~mL} / \mathrm{min}$.

$\mathrm{mAU}$

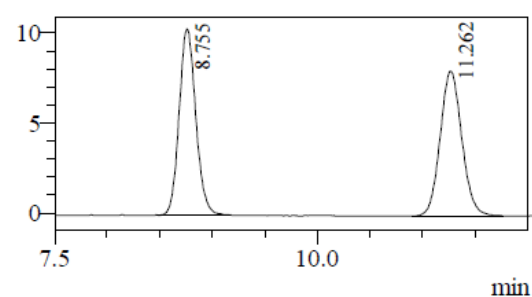

1 PDA Multi $1 / 220 \mathrm{~nm} 4 \mathrm{~nm}$

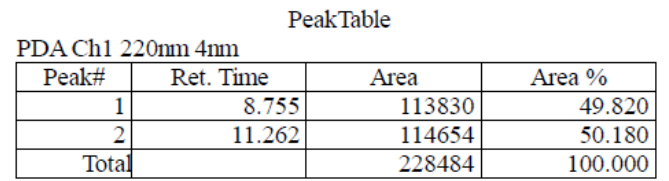

$\mathrm{mAU}$

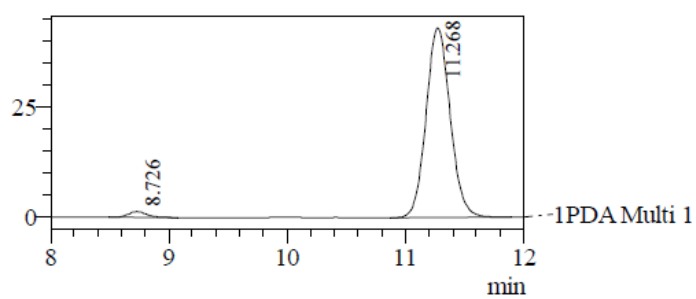

1 PDA Multi $1 / 220 \mathrm{~nm} 4 \mathrm{~nm}$

PDA Ch1 $220 \mathrm{~nm}$ 4nm
\begin{tabular}{|r|r|r|r|}
\hline Peak\# & Ret. Time & Area & \multicolumn{1}{|c|}{ Area $\%$} \\
\hline 1 & 8.726 & 14574 & 2.324 \\
\hline 2 & 11.268 & 612593 & 97.676 \\
\hline Total & & 627167 & 100.000 \\
\hline
\end{tabular}

${ }^{1} \mathrm{H}$ NMR $\left(400 \mathrm{MHz}, \mathrm{CDCl}_{3}\right): \delta 7.86(\mathrm{~d}, J=7.7 \mathrm{~Hz}, 1 \mathrm{H}), 7.63(\psi \mathrm{td}, J=7.5,1.1 \mathrm{~Hz}, 1 \mathrm{H}), 7.47(\psi \mathrm{t}, J=7.4 \mathrm{~Hz}$, $1 \mathrm{H}), 7.33(\mathrm{~d}, J=7.7 \mathrm{~Hz}, 1 \mathrm{H}), 7.25(\mathrm{~d}, J=7.4 \mathrm{~Hz}, 1 \mathrm{H}), 7.18(\psi \mathrm{td}, J=7.3,1.1 \mathrm{~Hz}, 1 \mathrm{H}), 7.12(\psi \mathrm{t}, J=7.6 \mathrm{~Hz}$, $1 \mathrm{H}), 6.80(\mathrm{~d}, J=6.9 \mathrm{~Hz}, 1 \mathrm{H}), 4.87(\mathrm{dd}, J=7.9,3.7 \mathrm{~Hz}, 1 \mathrm{H}), 3.29(\mathrm{dd}, J=19.1,8.1 \mathrm{~Hz}, 1 \mathrm{H}), 2.60(\mathrm{dd}, J=19.1$, $3.7 \mathrm{~Hz}, 1 \mathrm{H}), 2.46(\mathrm{~s}, 3 \mathrm{H})$.

${ }^{13} \mathrm{C}$ NMR $\left(101 \mathrm{MHz}, \mathrm{CDCl}_{3}\right): \delta 206.2,158.0,142.1,137.4,136.0,135.2,130.7,128.0,127.1,126.9,126.7$, $123.7,46.0,40.9,20.0$.

HRMS (ESI ${ }^{+}$): Calcd for $\mathrm{C}_{16} \mathrm{H}_{15} \mathrm{O}[\mathrm{M}+\mathrm{H}]^{+}:$223.1117. Found: 223.1113 .<smiles>COc1ccccc1C1CC(=O)c2ccccc21</smiles>

\section{(R)-3-(2-Methoxyphenyl)-1-indanone (4b) [1422961-12-3]}

The product was isolated by flash chromatography (ethyl acetate/hexanes 1:15) as yellow solid. $28.9 \mathrm{mg}, 61 \%$ yield. $97 \%$ ee.

$[\alpha]^{23} \mathrm{D}=+46.0^{\circ}\left(c=1.1, \mathrm{CHCl}_{3}\right)$. Literature value: $[\alpha]_{\mathrm{D}}=+23^{\circ}\left(c=0.5, \mathrm{CHCl}_{3}\right)$ for a sample of $84 \%$ ee $(R){ }^{4}$ HPLC: Daicel Chiralcel OJ-H, $n$-hexane/isopropanol 90/10, flow rate $=1.0 \mathrm{~mL} / \mathrm{min}$. 
mAU

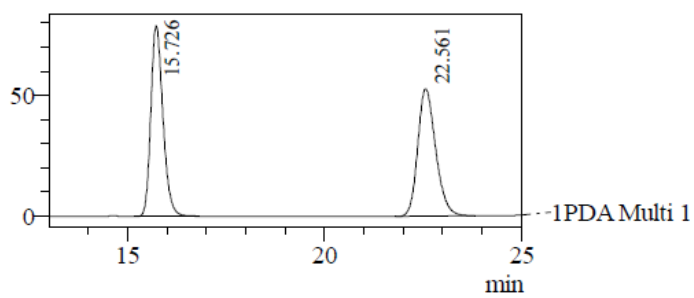

1 PDA Multi $1 / 220 \mathrm{~nm} 4 \mathrm{~nm}$

PDA Ch1 $220 \mathrm{~nm} 4 \mathrm{~nm}$
\begin{tabular}{|r|r|r|r|}
\hline Peak\# & Ret. Time & Area & \multicolumn{1}{c|}{ Area $\%$} \\
\hline 1 & 15.726 & 1682652 & 50.154 \\
\hline 2 & 22.561 & 1672335 & 49.846 \\
\hline Total & & 3354987 & 100.000 \\
\hline
\end{tabular}

mAU

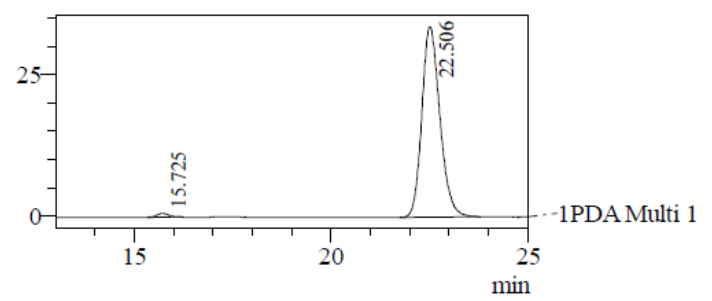

1 PDA Multi $1 / 220 \mathrm{~nm} 4 \mathrm{~nm}$

PDA Ch1 $220 \mathrm{~nm} 4 \mathrm{~nm}$
\begin{tabular}{|r|r|r|r|}
\hline Peak\# & Ret. Time & \multicolumn{1}{|c|}{ Area } & Area $\%$ \\
\hline 1 & 15.725 & 13852 & 1.274 \\
\hline 2 & 22.506 & 1073497 & 98.726 \\
\hline Total & & 1087349 & 100.000 \\
\hline
\end{tabular}

${ }^{1} \mathrm{H}$ NMR (400 MHz, $\left.\mathrm{CDCl}_{3}\right): \delta 7.81(\mathrm{~d}, J=7.7 \mathrm{~Hz}, 1 \mathrm{H}), 7.57(\psi \mathrm{td}, J=7.5,1.2 \mathrm{~Hz}, 1 \mathrm{H}), 7.42(\psi \mathrm{t}, J=7.5 \mathrm{~Hz}$, 1H), 7.28-7.25 (m, 1H), 7.22-7.19 (m, 2H), 7.07-7.02 (m, 2H), $4.54(\mathrm{dd}, J=8.0,3.8 \mathrm{~Hz}, 1 \mathrm{H}), 3.22(\mathrm{dd}, J=19.2$, $8.1 \mathrm{~Hz}, 1 \mathrm{H}), 2.67(\mathrm{dd}, J=19.2,3.9 \mathrm{~Hz}, 1 \mathrm{H}), 2.47(\mathrm{~s}, 3 \mathrm{H})$.

${ }^{13} \mathrm{C} \mathrm{NMR}\left(101 \mathrm{MHz}, \mathrm{CDCl}_{3}\right): \delta 206.0,157.9,140.8,137.2,136.9,135.3,128.3,128.1,127.4,127.0,123.6$, $46.9,44.1,16.1$.

MS (ESI): Calcd for $\mathrm{C}_{16} \mathrm{H}_{15} \mathrm{O}_{2}[\mathrm{M}+\mathrm{H}]^{+}: 239.11$. Found: 239.13.<smiles>Cc1cccc(C2CC(=O)c3ccccc32)c1</smiles>

\section{(S)-3-(m-Tolyl)-1-indanone (4c)}

The product was isolated by flash chromatography (ethyl acetate/hexanes 1:15) as yellow solid. $30.8 \mathrm{mg}, 69 \%$ yield. 95\% ee.

$[\alpha]^{23} \mathrm{D}=+33.2^{\circ}\left(c=0.85, \mathrm{CHCl}_{3}\right)$.

HPLC: Daicel Chiralcel OJ-H, $n$-hexane/isopropanol 95/5, flow rate $=1.0 \mathrm{~mL} / \mathrm{min}$.

mAU

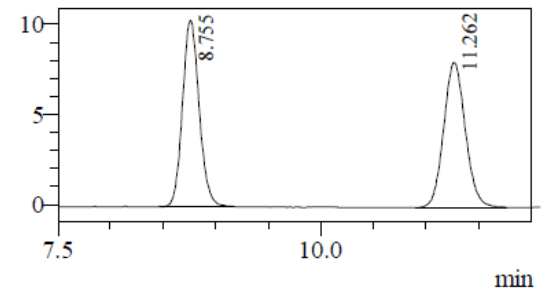

1 PDA Multi $1 / 220 \mathrm{~nm} 4 \mathrm{~nm}$

PDA Ch1 $220 \mathrm{~nm} 4 \mathrm{~nm}$
\begin{tabular}{|r|r|r|r|}
\hline \multicolumn{1}{|c|}{ Peak\# } & Ret. Time & Area & \multicolumn{1}{c|}{ Area $\%$} \\
\hline 1 & 8.755 & 113830 & 49.820 \\
\hline 2 & 11.262 & 114654 & 50.180 \\
\hline Total & & 228484 & 100.000 \\
\hline
\end{tabular}

$\mathrm{mAU}$

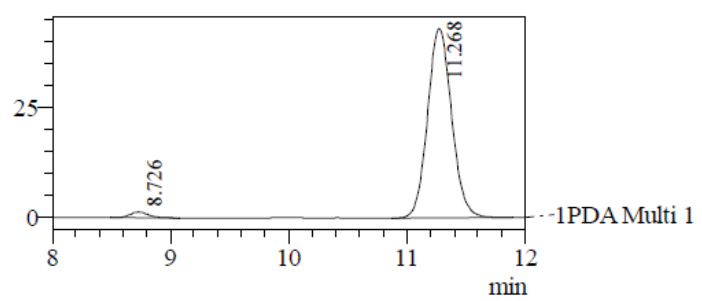

1 PDA Multi $1 / 220 \mathrm{~nm} 4 \mathrm{~nm}$

PeakTable

PDACh1 $220 \mathrm{~nm} 4 \mathrm{~nm}$
\begin{tabular}{|r|r|r|r|}
\hline Peak\# & Ret. Time & Area & Area $\%$ \\
\hline 1 & 8.726 & 14574 & 2.324 \\
\hline 2 & 11.268 & 612593 & 97.676 \\
\hline Total & & 627167 & 100.000 \\
\hline
\end{tabular}


${ }^{1} \mathrm{H}$ NMR (400 MHz, $\left.\mathrm{CDCl}_{3}\right): \delta 7.81(\mathrm{~d}, J=7.6 \mathrm{~Hz}, 1 \mathrm{H}), 7.57(\psi \mathrm{td}, J=7.6,1.2 \mathrm{~Hz}, 1 \mathrm{H}), 7.41(\psi \mathrm{t}, J=7.5 \mathrm{~Hz}$, 1H), 7.29- $7.26(\mathrm{~m}, 1 \mathrm{H}), 7.23-7.17(\mathrm{~m}, 1 \mathrm{H}), 7.06$ (d, $J=7.4 \mathrm{~Hz}, 1 \mathrm{H}), 6.93-6.91(\mathrm{~m}, 2 \mathrm{H}), 4.54(\mathrm{dd}, J=8.0,3.8$ $\mathrm{Hz}, 1 \mathrm{H}), 3.20$ (dd, $J=19.2,8.0 \mathrm{~Hz}, 1 \mathrm{H}), 2.69$ (dd, $J=19.2,3.8 \mathrm{~Hz}, 1 \mathrm{H}), 2.31$ (s, 3H).

${ }^{13} \mathrm{C} \mathrm{NMR}\left(101 \mathrm{MHz}, \mathrm{CDCl}_{3}\right): \delta 206.3,158.2,143.8,138.8,136.9,135.2,128.9,128.5,128.0,127.9,127.0$, $124.8,123.5,47.0,44.6,21.6$.

HRMS (ESI ${ }^{+}$: Calcd for $\mathrm{C}_{16} \mathrm{H}_{15} \mathrm{O}[\mathrm{M}+\mathrm{H}]^{+}: 223.1117$. Found: 223.1127.

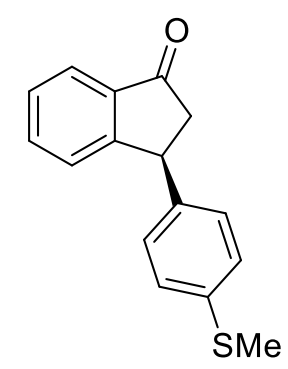

\section{(S)-3-(4-Methylthiophenyl)-1-indanone (4d)}

The product was isolated by flash chromatography (ethyl acetate/hexanes 1:15) as yellow solid. $31.6 \mathrm{mg}, 62 \%$ yield. $97 \%$ ee.

$[\alpha]^{23} \mathrm{D}=-17.0^{\circ}\left(c=0.85, \mathrm{CHCl}_{3}\right)$.

HPLC: Daicel Chiralcel OJ-H, $n$-hexane/isopropanol 95/5, flow rate $=1.0 \mathrm{~mL} / \mathrm{min}$.

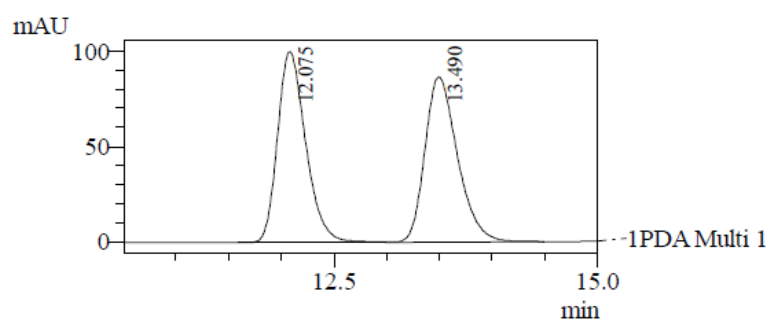

1 PDA Multi $1 / 220 \mathrm{~nm} 4 \mathrm{~nm}$

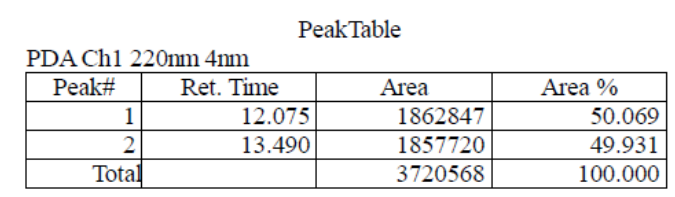

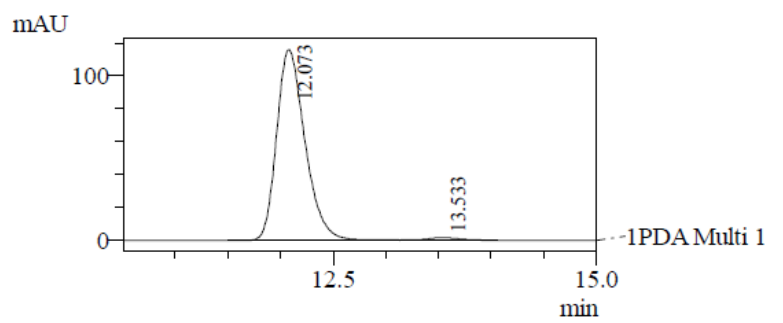

1 PDA Multi $1 / 220 \mathrm{~nm} 4 \mathrm{~nm}$

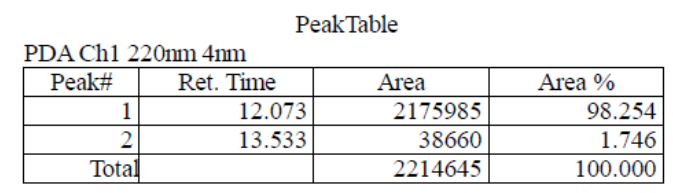

${ }^{1} \mathrm{H}$ NMR (400 MHz, $\left.\mathrm{CDCl}_{3}\right): \delta 7.80(\mathrm{~d}, J=7.6 \mathrm{~Hz}, 1 \mathrm{H}), 7.55(\psi \mathrm{t}, J=7.5 \mathrm{~Hz}, 1 \mathrm{H}), 7.39(\psi \mathrm{t}, J=7.4 \mathrm{~Hz}, 1 \mathrm{H})$, $7.30(\mathrm{~d}, J=7.7 \mathrm{~Hz}, 1 \mathrm{H}), 7.26-7.20(\mathrm{~m}, 1 \mathrm{H}), 6.98-6.93(\mathrm{~m}, 1 \mathrm{H}), 6.90-6.85(\mathrm{~m}, 2 \mathrm{H}), 4.89(\mathrm{dd}, J=8.0,3.3 \mathrm{~Hz}$, 1H), 3.75 (s, 3H), 3.17 (dd, $J=19.1,8.1 \mathrm{~Hz}, 1 \mathrm{H}), 2.69$ (dd, $J=19.1,3.6 \mathrm{~Hz}, 1 \mathrm{H})$.

${ }^{13} \mathrm{C} \mathrm{NMR}\left(101 \mathrm{MHz}, \mathrm{CDCl}_{3}\right): \delta 206.8,158.0,157.4,137.3,134.8,131.8,128.5,128.3,127.6,126.8,123.4$, $120.9,111.1,55.5,45.4,39.3$.

HRMS (ESI ${ }^{+}$): Calcd for $\mathrm{C}_{16} \mathrm{H}_{15} \mathrm{OS}[\mathrm{M}+\mathrm{H}]^{+}: 255.0838$. Found: 255.0836 . 
<smiles>COc1ccc(C2CC(=O)c3ccccc32)c(OC)c1</smiles>

\section{(R)-3-(2,4-Dimethoxyphenyl)-1-indanone (4e)}

The product was isolated by flash chromatography (ethyl acetate/hexanes 1:15) as yellow solid. $38.6 \mathrm{mg}, 72 \%$ yield. $96 \%$ ee.

$[\alpha]^{23}{ }_{\mathrm{D}}=+21.8^{\circ}\left(c=1.0, \mathrm{CHCl}_{3}\right)$.

HPLC: Daicel Chiralcel IC-3, $n$-hexane/isopropanol 95/5, flow rate $=1.0 \mathrm{~mL} / \mathrm{min}$.

$\mathrm{mAU}$

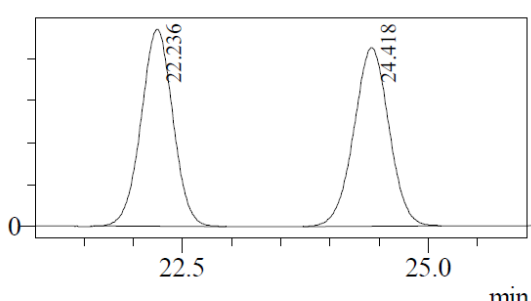

1 PDA Multi $1 / 220 \mathrm{~nm} 4 \mathrm{~nm}$

PDA Ch1 220nm 4nm
\begin{tabular}{|r|r|r|r|}
\hline \multicolumn{1}{|c|}{ Peak\# } & Ret. Time & \multicolumn{1}{c|}{ Area } & Area $\%$ \\
\hline 1 & 22.236 & 528595 & 50.009 \\
\hline 2 & 24.418 & 528409 & 49.991 \\
\hline Total & & 1057004 & 100.000 \\
\hline
\end{tabular}

$\mathrm{mAU}$

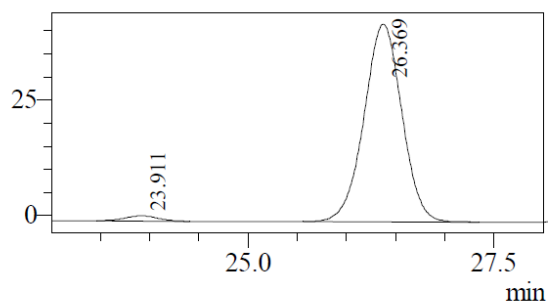

1 PDA Multi 1/220nm 4nm

${ }^{1} \mathrm{H} \mathrm{NMR}\left(400 \mathrm{MHz}, \mathrm{CDCl}_{3}\right): \delta 7.78(\mathrm{~d}, J=7.6 \mathrm{~Hz}, 1 \mathrm{H}), 7.54(\psi \mathrm{td}, J=7.5,1.2 \mathrm{~Hz}, 1 \mathrm{H}), 7.37(\psi \mathrm{t}, J=7.3 \mathrm{~Hz}$, $1 \mathrm{H}), 7.29(\mathrm{~d}, J=7.7 \mathrm{~Hz}, 1 \mathrm{H}), 6.85(\mathrm{~d}, J=8.4 \mathrm{~Hz}, 1 \mathrm{H}), 6.46(\mathrm{~d}, J=2.4 \mathrm{~Hz}, 1 \mathrm{H}), 6.39(\mathrm{dd}, J=8.4,2.4 \mathrm{~Hz}, 1 \mathrm{H})$, $4.80(\mathrm{dd}, J=8.0,3.5 \mathrm{~Hz}, 1 \mathrm{H}), 3.78$ (s, 3H), 3.72 (s, 3H), $3.14(\mathrm{dd}, J=19.1,8.1 \mathrm{~Hz}, 1 \mathrm{H}), 2.65(\mathrm{dd}, J=19.1,3.6$ $\mathrm{Hz}, 1 \mathrm{H})$.

${ }^{13} \mathrm{C} \mathrm{NMR}\left(101 \mathrm{MHz}, \mathrm{CDCl}_{3}\right): \delta 207.0,160.0,158.34,158.29,137.2,134.7,128.9,127.5,126.7,124.3,123.3$, $104.3,99.1,55.5,45.5,38.8$.

HRMS (ESI ${ }^{+}$): Calcd for $\mathrm{C}_{17} \mathrm{H}_{17} \mathrm{O}_{3}[\mathrm{M}+\mathrm{H}]^{+}:$269.1172. Found: 269.1181 .<smiles>COc1ccc(C2CC(=O)c3ccccc32)cc1OC</smiles> 
The product was isolated by flash chromatography (ethyl acetate/hexanes 1:10) as yellow solid. $34.6 \mathrm{mg}, 65 \%$ yield. $96 \%$ ee.

$[\alpha]^{23} \mathrm{D}=-12.6^{\circ}\left(c=1.0, \mathrm{CHCl}_{3}\right)$.

HPLC: Daicel Chiralcel AD-H, $n$-hexane/isopropanol 98/2, flow rate $=0.5 \mathrm{~mL} / \mathrm{min}$.

$\mathrm{mAU}$

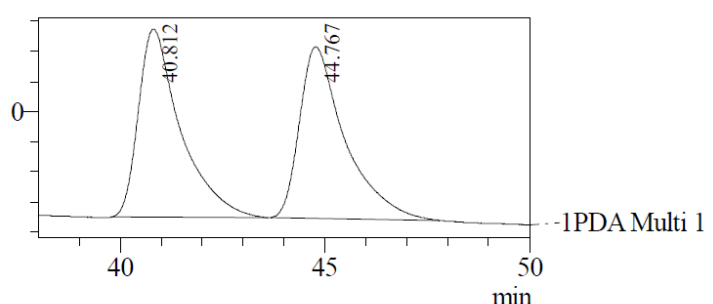

1 PDA Multi $1 / 220 \mathrm{~nm} 4 \mathrm{~nm}$

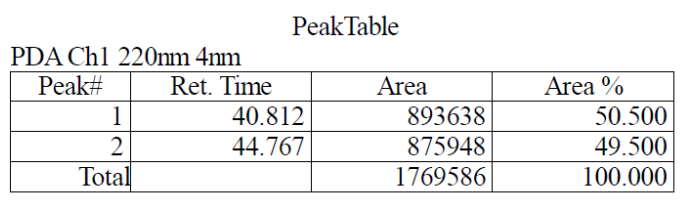

$\mathrm{mAU}$

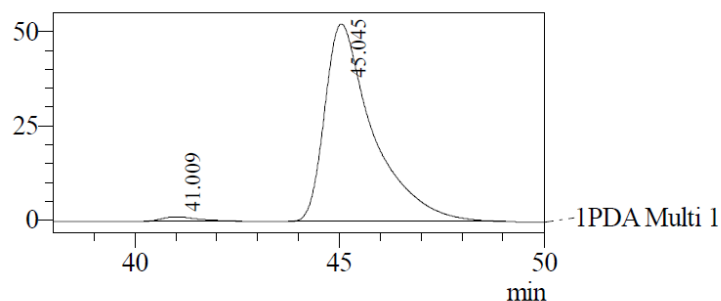

1 PDA Multi $1 / 220 \mathrm{~nm} 4 \mathrm{~nm}$

PeakTable
\begin{tabular}{|r|r|r|r|}
\hline PDA Ch1 220nm 4nm & Area & Area $\%$ \\
\hline 1 & Ret. Time & Area & 1.781 \\
\hline 2 & 41.009 & 77986 & 1.045 \\
\hline Total & & 4300781 & 98.219 \\
\hline
\end{tabular}

${ }^{1} \mathrm{H} \mathrm{NMR}\left(400 \mathrm{MHz}, \mathrm{CDCl}_{3}\right): \delta 7.82(\mathrm{~d}, J=7.7 \mathrm{~Hz}, 1 \mathrm{H}), 7.61(\psi \mathrm{td}, J=7.6,1.1 \mathrm{~Hz}, 1 \mathrm{H}), 7.44(\psi \mathrm{t}, J=7.5 \mathrm{~Hz}$, 1H), $7.35(\mathrm{~d}, J=7.7 \mathrm{~Hz}, 1 \mathrm{H}), 7.27(\mathrm{~s}, 1 \mathrm{H}), 6.72(\mathrm{~d}, J=8.7 \mathrm{~Hz}, 1 \mathrm{H}), 6.56$ (br s, 1H), 5.05 (br s, $1 \mathrm{H}), 3.91(\mathrm{~s}$, $3 \mathrm{H}), 3.84$ (s, 3H), 3.28 (dd, $J=19.2,8.1 \mathrm{~Hz}, 1 \mathrm{H}), 2.58$ (d, $J=18.6 \mathrm{~Hz}, 1 \mathrm{H})$.

${ }^{13} \mathrm{C} \mathrm{NMR}\left(101 \mathrm{MHz}, \mathrm{CDCl}_{3}\right): \delta 205.8,156.9,152.7,145.8,137.4,135.1,134.5,128.8,128.1,127.0,123.7$, 123.1, 111.0, 60.8, 56.2, 45.8, 40.8 .

HRMS (ESI ${ }^{+}$: Calcd for $\mathrm{C}_{17} \mathrm{H}_{17} \mathrm{O}_{3}[\mathrm{M}+\mathrm{H}]^{+}:$269.1172. Found: 269.1179 .<smiles>COc1cc(C2CC(=O)c3ccccc32)ccc1F</smiles>

\section{(S)-3-(4-Fluoro-3-methoxyphenyl)-1-indanone (4g)}

The product was isolated by flash chromatography (ethyl acetate/hexanes 1:15) as yellow solid. 38.6 mg, 75\% yield. $93 \%$ ee.

$[\alpha]^{23} \mathrm{D}=+22.2^{\circ}\left(c=1.0, \mathrm{CHCl}_{3}\right)$.

HPLC: Daicel Chiralcel OD-H, $n$-hexane/isopropanol 95/5, flow rate $=1.0 \mathrm{~mL} / \mathrm{min}$. 


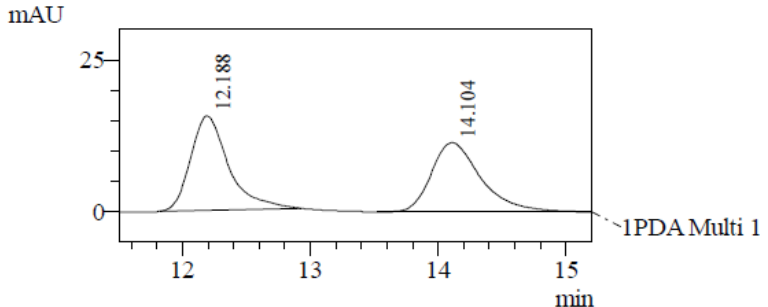

1 PDA Multi $1 / 220 \mathrm{~nm} 4 \mathrm{~nm}$

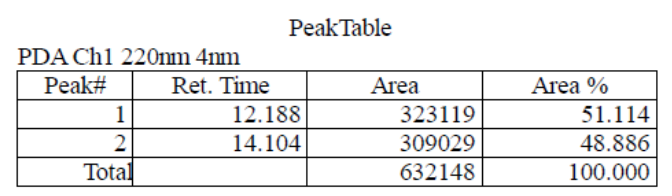

$\mathrm{mAU}$

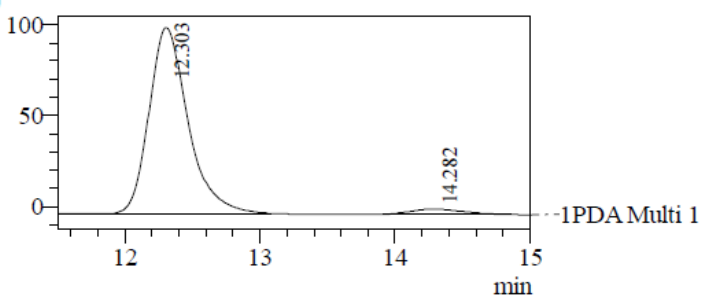

1 PDA Multi $1 / 220 \mathrm{~nm} 4 \mathrm{~nm}$

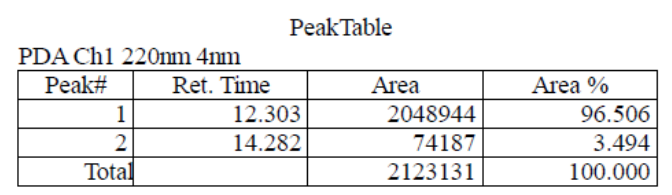

${ }^{1} \mathrm{H}$ NMR (400 MHz, $\left.\mathrm{CDCl}_{3}\right): \delta 7.82(\mathrm{~d}, J=7.7 \mathrm{~Hz}, 1 \mathrm{H}), 7.62-7.58(\mathrm{~m}, 1 \mathrm{H}), 7.44(\psi \mathrm{t}, J=7.4 \mathrm{~Hz}, 1 \mathrm{H}), 7.27(\mathrm{~d}, J$ $=7.7 \mathrm{~Hz}, 1 \mathrm{H}), 7.03-6.98(\mathrm{~m}, 1 \mathrm{H}), 6.68-6.64(\mathrm{~m}, 2 \mathrm{H}), 4.54(\mathrm{dd}, J=8.0,3.8 \mathrm{~Hz}, 1 \mathrm{H}), 3.82(\mathrm{~s}, 3 \mathrm{H}), 3.24(\mathrm{dd}, J=$ $19.2,8.1 \mathrm{~Hz}, 1 \mathrm{H}), 2.64(\mathrm{dd}, J=19.2,3.9 \mathrm{~Hz}, 1 \mathrm{H})$.

${ }^{13} \mathrm{C}$ NMR $\left(101 \mathrm{MHz}, \mathrm{CDCl}_{3}\right): \delta 205.7,157.6,151.3\left(\mathrm{~d}, J_{C-F}=245.3 \mathrm{~Hz}\right), 148.1\left(\mathrm{~d}, J_{C-F}=9.9 \mathrm{~Hz}\right), 140.2\left(\mathrm{~d}, J_{C-F}\right.$ $=3.8 \mathrm{~Hz}), 136.9,135.3,128.2,126.9,123.7,120.1\left(\mathrm{~d}, J_{C-F}=6.9 \mathrm{~Hz}\right), 116.4\left(\mathrm{~d}, J_{C-F}=18.4 \mathrm{~Hz}\right), 112.9\left(\mathrm{~d}, J_{C-F}=\right.$ $1.8 \mathrm{~Hz}), 56.5,47.0,44.3$.

${ }^{19} \mathrm{~F} \mathrm{NMR}\left(282 \mathrm{MHz}, \mathrm{CDCl}_{3}\right): \delta-137.4$.

HRMS (ESI ${ }^{+}$): Calcd for $\mathrm{C}_{16} \mathrm{H}_{14} \mathrm{FO}_{2}[\mathrm{M}+\mathrm{H}]^{+}:$257.0972. Found: 257.0989.<smiles>O=C1CC(c2ccc(Cl)c(Cl)c2)c2ccccc21</smiles>

\section{(S)-3-(3,4-Dichlorophenyl)-1-indanone (4h) [477332-35-7]}

The product was isolated by flash chromatography (ethyl acetate/hexanes 1:15) as yellow solid. $36.0 \mathrm{mg}, 65 \%$ yield. $92 \%$ ee.

$[\alpha]^{23}{ }_{\mathrm{D}}=+48^{\circ}\left(c=1.0, \mathrm{CHCl}_{3}\right)$. Literature value: $[\alpha]^{24}{ }_{\mathrm{D}}=+49.5^{\circ}\left(c=1.33, \mathrm{CHCl}_{3}\right)$ for a sample of $98 \%$ ee $(S) .{ }^{4}$ HPLC: Daicel Chiralcel IC-3, $n$-hexane/isopropanol 95/5, flow rate $=1.0 \mathrm{~mL} / \mathrm{min}$. 
mAU

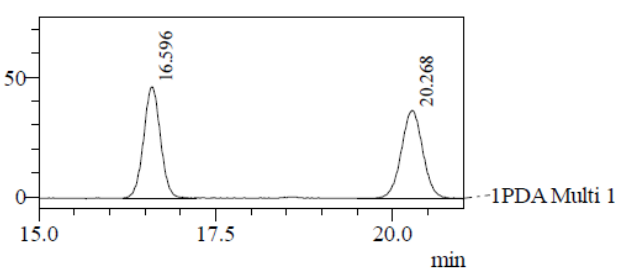

1 PDA Multi $1 / 200 \mathrm{~nm} 4 \mathrm{~nm}$

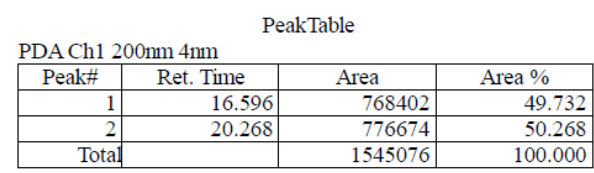

mAU

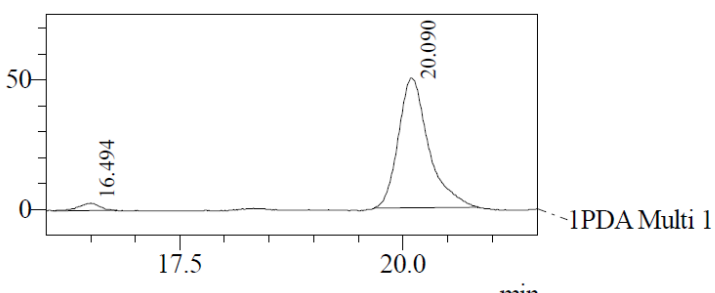

$1 \mathrm{I}$

PeakTable

PDA Chl 200nm 4nm
\begin{tabular}{|r|r|r|r|}
\hline \multicolumn{1}{|c|}{ Peak\# $\#$ Ret. Time } & Area & \multicolumn{1}{c|}{ Area $\%$} \\
\hline 1 & 16.494 & 45896 & 3.756 \\
\hline 2 & 20.090 & 1175998 & 96.244 \\
\hline Total & & 1221894 & 100.000 \\
\hline
\end{tabular}

${ }^{1} \mathrm{H}$ NMR $\left(400 \mathrm{MHz}, \mathrm{CDCl}_{3}\right): \delta 7.83(\mathrm{~d}, J=7.7 \mathrm{~Hz}, 1 \mathrm{H}), 7.61(\psi \mathrm{td}, J=7.6,1.2 \mathrm{~Hz}, 1 \mathrm{H}), 7.46(\psi \mathrm{t}, J=7.5 \mathrm{~Hz}$, $1 \mathrm{H}), 7.38(\mathrm{~d}, J=8.3 \mathrm{~Hz}, 1 \mathrm{H}), 7.29-7.25(\mathrm{~m}, 1 \mathrm{H}), 7.23(\mathrm{~d}, J=2.1 \mathrm{~Hz}, 1 \mathrm{H}), 6.95(\mathrm{dd}, J=8.3,2.1 \mathrm{~Hz}, 1 \mathrm{H}), 4.55$

$(\mathrm{dd}, J=8.1,3.8 \mathrm{~Hz}, 1 \mathrm{H}), 3.23(\mathrm{dd}, J=19.2,8.1 \mathrm{~Hz}, 1 \mathrm{H}), 2.62(\mathrm{dd}, J=19.2,3.9 \mathrm{~Hz}, 1 \mathrm{H})$.

${ }^{13} \mathrm{C}$ NMR $\left(101 \mathrm{MHz}, \mathrm{CDCl}_{3}\right): \delta 205.0,156.7,144.1,136.9,135.5,133.1,131.3,131.1,129.8,128.5,127.1$, $126.8,123.9,46.6,43.7$.

MS (ESI ${ }^{+}$): Calcd for $\mathrm{C}_{15} \mathrm{H}_{11} \mathrm{Cl}_{2} \mathrm{O}[\mathrm{M}+\mathrm{H}]^{+}:$277.02. Found: 277.12 .<smiles>O=C1CC(c2ccc(F)c(Cl)c2)c2ccccc21</smiles>

\section{(S)-3-(3-Chloro-4-fluorophenyl)-1-indanone (4i)}

The product was isolated by flash chromatography (ethyl acetate/hexanes 1:15) as yellow solid. $42.5 \mathrm{mg}, 82 \%$ yield. $91 \%$ ee.

$[\alpha]^{23} \mathrm{D}=+43.9^{\circ}\left(c=0.65, \mathrm{CHCl}_{3}\right)$.

HPLC: Daicel Chiralcel OD-H, $n$-hexane/isopropanol 95/5, flow rate $=1.0 \mathrm{~mL} / \mathrm{min}$.

mAU

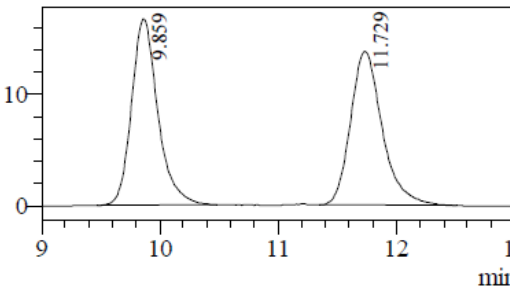

1. PDA Multi $1 / 220 \mathrm{~nm} 4 \mathrm{~nm}$

PDACh1 $220 \mathrm{~nm} 4 \mathrm{~nm}$
\begin{tabular}{|r|r|r|r|}
\hline Peak\# & Ret. Time & \multicolumn{1}{|c|}{ Area } & Area $\%$ \\
\hline 1 & 9.859 & 258599 & 50.198 \\
\hline 2 & 11.729 & 256562 & 49.802 \\
\hline Total & & 515161 & 100.000 \\
\hline
\end{tabular}

mAU

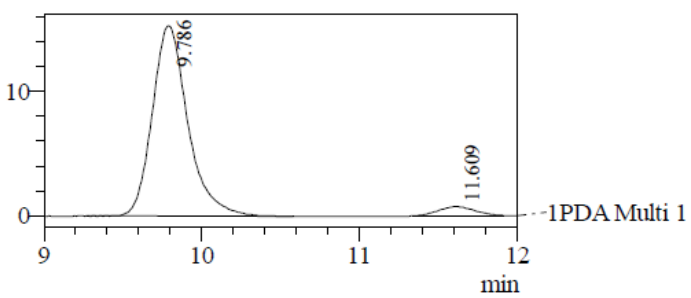

1 PDA Multi $1 / 220 \mathrm{~nm} 4 \mathrm{~nm}$

PeakTable

PDA Ch1 $220 \mathrm{~nm} 4 \mathrm{~nm}$
\begin{tabular}{|r|r|r|r|}
\hline Peak\# & Ret. Time & \multicolumn{1}{|c|}{ Area } & Area $\%$ \\
\hline 1 & 9.786 & 239703 & 95.366 \\
\hline 2 & 11.609 & 11647 & 4.634 \\
\hline Total & & 251350 & 100.000 \\
\hline
\end{tabular}


${ }^{1} \mathrm{H}$ NMR $\left(400 \mathrm{MHz}, \mathrm{CDCl}_{3}\right): \delta 7.83(\mathrm{~d}, J=7.7 \mathrm{~Hz}, 1 \mathrm{H}), 7.61(\psi \mathrm{td}, J=7.5,1.2 \mathrm{~Hz}, 1 \mathrm{H}), 7.46(\psi \mathrm{t}, J=7.5 \mathrm{~Hz}$, $1 \mathrm{H}), 7.28-7.25(\mathrm{~m}, 1 \mathrm{H}), 7.16(\mathrm{dd}, J=6.9,2.2 \mathrm{~Hz}, 1 \mathrm{H}), 7.11(\psi \mathrm{t}, J=8.6 \mathrm{~Hz}, 1 \mathrm{H}), 7.00-6.98(\mathrm{~m}, 1 \mathrm{H}), 4.57(\mathrm{dd}, J$ $=8.0,3.8 \mathrm{~Hz}, 1 \mathrm{H}), 3.25(\mathrm{dd}, J=19.2,8.1 \mathrm{~Hz}, 1 \mathrm{H}), 2.64(\mathrm{dd}, J=19.2,3.9 \mathrm{~Hz}, 1 \mathrm{H})$.

${ }^{13} \mathrm{C} \mathrm{NMR}\left(101 \mathrm{MHz}, \mathrm{CDCl}_{3}\right): \delta 205.1,157.2\left(\mathrm{~d}, J_{C-F}=248.5 \mathrm{~Hz}\right), 156.9,141.0\left(\mathrm{~d}, J_{C-F}=3.9 \mathrm{~Hz}\right), 136.9,135.5$, 129. $9\left(\mathrm{~d}, J_{C-F}=0.8 \mathrm{~Hz}\right), 128.5,127.4\left(\mathrm{~d}, J_{C-F}=7.1 \mathrm{~Hz}\right), 126.9,123.8,121.6\left(\mathrm{~d}, J_{C-F}=17.9 \mathrm{~Hz}\right), 117.2\left(\mathrm{~d}, J_{C-F}=\right.$ $21.3 \mathrm{~Hz}), 46.8,43.7$.

${ }^{19} \mathrm{~F}$ NMR $\left(282 \mathrm{MHz}, \mathrm{CDCl}_{3}\right): \delta-117.8$.

HRMS (ESI ${ }^{+}$): Calcd for $\mathrm{C}_{15} \mathrm{H}_{11} \mathrm{ClFO}[\mathrm{M}+\mathrm{H}]^{+}: 261.0477$. Found: 261.0481 .

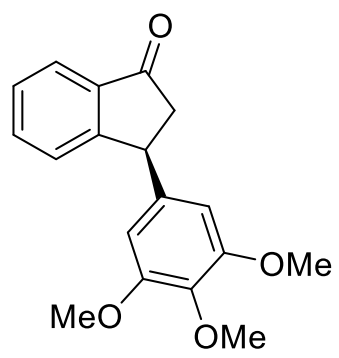

\section{(S)-3-(3,4,5-Trimethoxyphenyl)-1-indanone $(4 \mathbf{j})$}

The product was isolated by flash chromatography (ethyl acetate/hexanes 1:5) as yellow solid. $38.7 \mathrm{mg}, 65 \%$ yield. $87 \%$ ee.

$[\alpha]^{23} \mathrm{D}=+68.7^{\circ}\left(c=1.0, \mathrm{CHCl}_{3}\right)$.

HPLC: Daicel Chiralcel OJ-H, $n$-hexane/isopropanol 80/20, flow rate $=1.0 \mathrm{~mL} / \mathrm{min}$.

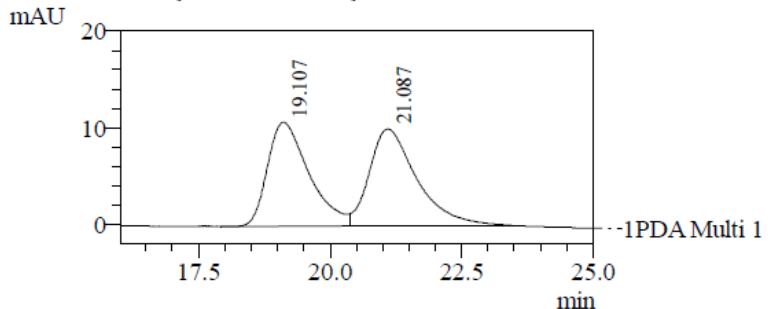

1 PDA Multi $1 / 220 \mathrm{~nm} 4 \mathrm{~nm}$

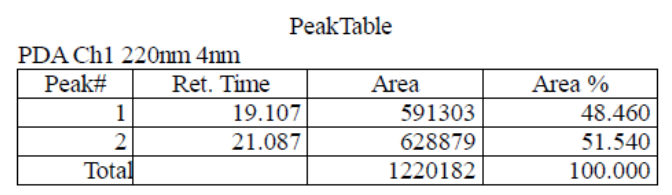

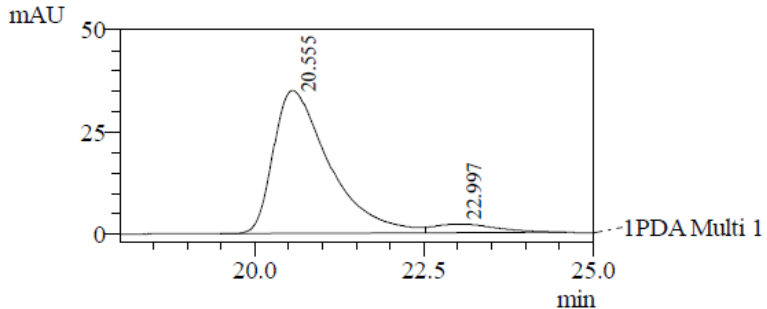

1 PDA Multi $1 / 220 \mathrm{~nm} 4 \mathrm{~nm}$

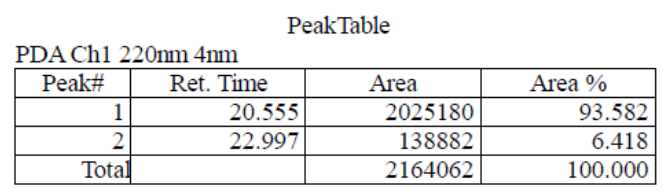

${ }^{1} \mathrm{H}$ NMR (400 MHz, $\left.\mathrm{CDCl}_{3}\right): \delta 7.82(\mathrm{~d}, J=7.7 \mathrm{~Hz}, 1 \mathrm{H}), 7.60(\psi \mathrm{t}, J=7.4 \mathrm{~Hz}, 1 \mathrm{H}), 7.44(\psi \mathrm{t}, J=7.4 \mathrm{~Hz}, 1 \mathrm{H})$, $7.33(\mathrm{~d}, J=7.7 \mathrm{~Hz}, 1 \mathrm{H}), 6.32(\mathrm{~s}, 2 \mathrm{H}), 4.51(\mathrm{dd}, J=7.9,3.8 \mathrm{~Hz}, 1 \mathrm{H}), 3.83(\mathrm{~s}, 3 \mathrm{H}), 3.79(\mathrm{~s}, 6 \mathrm{H}), 3.22(\mathrm{dd}, J=$ 19.2, $8.0 \mathrm{~Hz}, 1 \mathrm{H}), 2.70(\mathrm{dd}, J=19.2,3.9 \mathrm{~Hz}, 1 \mathrm{H})$.

${ }^{13} \mathrm{C} \mathrm{NMR}\left(75 \mathrm{MHz}, \mathrm{CDCl}_{3}\right): \delta 206.0,157.8,153.7,139.4,137.1,136.8,135.2,128.1,127.0,123.5,104.8,61.0$, $56.3,46.9,44.9$.

HRMS (ESI ${ }^{+}$: Calcd for $\mathrm{C}_{18} \mathrm{H}_{18} \mathrm{NaO}_{4}[\mathrm{M}+\mathrm{Na}]^{+}: 321.1097$. Found: 321.1109 . 
<smiles>O=C1CC(c2ccsc2)c2ccccc21</smiles>

\section{(R)-3-(Thiophen-3-yl)-1-indanone (4k)}

The product was isolated by flash chromatography (ethyl acetate/hexanes 1:15) as yellow solid. $28.8 \mathrm{mg}, 67 \%$ yield. $97 \%$ ee.

$[\alpha]^{23} \mathrm{D}=+3.5^{\circ}\left(c=0.52, \mathrm{CHCl}_{3}\right)$. Literature value: $[\alpha]^{23} \mathrm{D}=-12^{\circ}\left(c=0.15, \mathrm{CHCl}_{3}\right)$ for a sample of its enantiomer in $94 \%$ ee $(S){ }^{4}$

HPLC: Daicel Chiralcel IC-3, $n$-hexane/isopropanol 95/5, flow rate $=1.0 \mathrm{~mL} / \mathrm{min}$.

$\mathrm{mAU}$

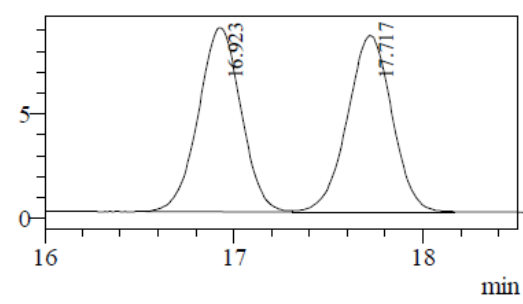

1 PDA Multi $1 / 220 \mathrm{~mm} 4 \mathrm{~nm}$

PeakTable
\begin{tabular}{|r|r|r|r|}
\hline Peak\# $\# 1220 \mathrm{~nm} 4 \mathrm{~nm}$ & Ret. Time & Area & \multicolumn{1}{|c|}{ Area $\%$} \\
\hline 1 & 16.923 & 138506 & 50.033 \\
\hline 2 & 17.717 & 138323 & 49.967 \\
\hline Total & & 276829 & 100.000 \\
\hline
\end{tabular}

$\mathrm{mAU}$

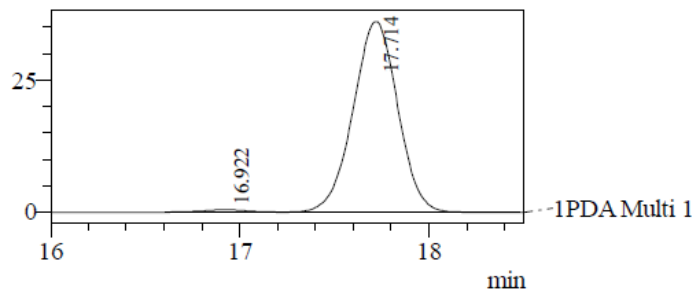

1 PDA Multi 1 / 220nm 4nm

PDA Ch1 $220 \mathrm{~nm} 4 \mathrm{~nm}$
\begin{tabular}{|r|r|r|r|}
\hline \multicolumn{1}{|c|}{ Peak\# } & Ret. Time & \multicolumn{1}{|c|}{ Area } & \multicolumn{1}{c|}{ Area $\%$} \\
\hline 1 & 16.922 & 7800 & 1.295 \\
\hline 2 & 17.714 & 594574 & 98.705 \\
\hline Total & & 602374 & 100.000 \\
\hline
\end{tabular}

${ }^{1} \mathrm{H}$ NMR (300 MHz, $\left.\mathrm{CDCl}_{3}\right): \delta 7.80(\mathrm{~d}, J=7.6 \mathrm{~Hz}, 1 \mathrm{H}), 7.60(\psi \mathrm{t}, J=7.5 \mathrm{~Hz}, 1 \mathrm{H}), 7.43-7.35(\mathrm{~m}, 2 \mathrm{H}), 7.29$ (dd, $J=5.0,3.0 \mathrm{~Hz}, 1 \mathrm{H}), 7.04-7.03(\mathrm{~m}, 1 \mathrm{H}), 6.81(\mathrm{~d}, J=5.0 \mathrm{~Hz}, 1 \mathrm{H}), 4.71(\mathrm{dd}, J=7.9,3.7 \mathrm{~Hz}, 1 \mathrm{H}), 3.20(\mathrm{dd}, J=$ $19.1,8.0 \mathrm{~Hz}, 1 \mathrm{H}), 2.70(\mathrm{dd}, J=19.1,3.8 \mathrm{~Hz}, 1 \mathrm{H})$.

${ }^{13} \mathrm{C}$ NMR (101 MHz, $\left.\mathrm{CDCl}_{3}\right): \delta 205.8,157.4,144.0,136.6,135.2,128.1,126.86,126.85,126.7,123.7,121.3$, 46.1, 39.7.

HRMS (ESI ${ }^{+}$): Calcd for $\mathrm{C}_{13} \mathrm{H}_{11} \mathrm{OS}[\mathrm{M}+\mathrm{H}]^{+}: 215.0525$. Found: 215.0535 .<smiles>O=C1CC(c2ccoc2)c2ccccc21</smiles>

\section{(R)-3-(Furan-3-yl)-1-indanone (4I)}

The product was isolated by flash chromatography (ethyl acetate/hexanes 1:15) as yellow solid. $25.6 \mathrm{mg}, 65 \%$ yield. $94 \%$ ee. 
$[\alpha]^{23}{ }_{\mathrm{D}}=-5.9^{\circ}\left(c=0.48, \mathrm{CHCl}_{3}\right)$. Literature value: $[\alpha]^{23} \mathrm{D}=+13^{\circ}\left(c=1, \mathrm{CHCl}_{3}\right)$ for a sample of its enantiomer in $97 \%$ ee $(S){ }^{4}$

HPLC: Daicel Chiralcel OJ-H, $n$-hexane/isopropanol 95/5, flow rate $=1.0 \mathrm{~mL} / \mathrm{min}$.

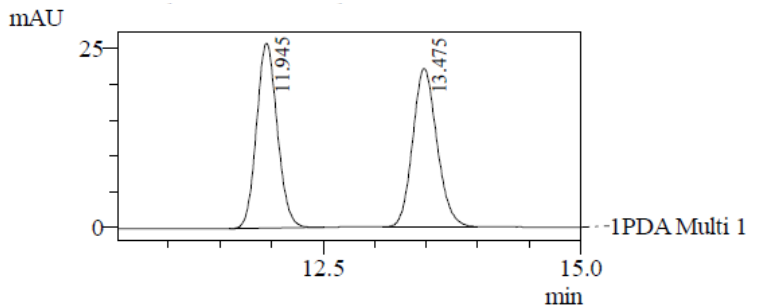

1 PDA Multi $1 / 220 \mathrm{~nm} 4 \mathrm{~nm}$

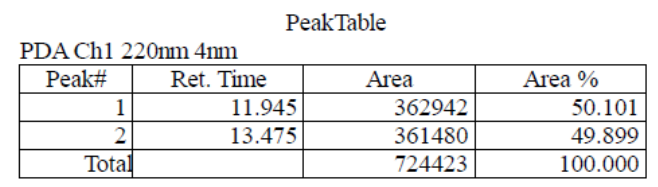

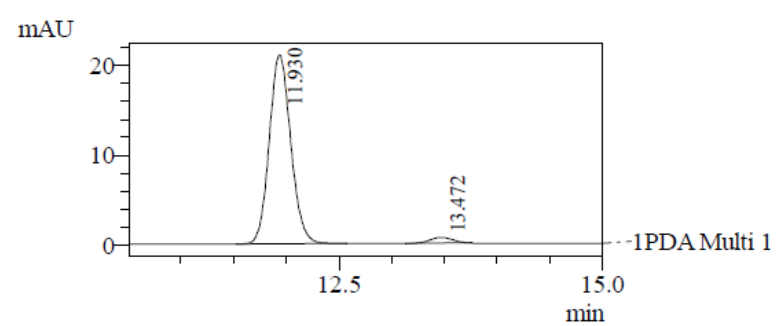

1 PDA Multi $1 / 220 \mathrm{~nm} 4 \mathrm{~nm}$

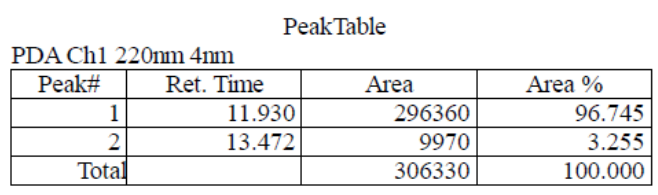

${ }^{1} \mathrm{H}$ NMR (400 MHz, $\left.\mathrm{CDCl}_{3}\right): \delta 7.80(\mathrm{~d}, J=7.6 \mathrm{~Hz}, 1 \mathrm{H}), 7.65-7.59(\mathrm{~m}, 1 \mathrm{H}), 7.52(\mathrm{dd}, J=7.7,0.8 \mathrm{~Hz}, 1 \mathrm{H}), 7.44$

$(\psi \mathrm{t}, J=7.4 \mathrm{~Hz}, 1 \mathrm{H}), 7.35(\mathrm{dd}, J=1.8,0.8 \mathrm{~Hz}, 1 \mathrm{H}), 6.32(\mathrm{dd}, J=3.1,1.9 \mathrm{~Hz}, 1 \mathrm{H}), 6.11(\mathrm{~d}, J=3.2 \mathrm{~Hz}, 1 \mathrm{H}), 4.69$

(dd, $J=8.0,4.0 \mathrm{~Hz}, 1 \mathrm{H}), 3.13(\mathrm{dd}, J=19.0,8.0 \mathrm{~Hz}, 1 \mathrm{H}), 2.88(\mathrm{dd}, J=19.0,4.1 \mathrm{~Hz}, 1 \mathrm{H})$.

${ }^{13} \mathrm{C} \mathrm{NMR}\left(101 \mathrm{MHz}, \mathrm{CDCl}_{3}\right): \delta 205.0,155.3,154.8,142.3,136.6,135.1,128.4,126.7,123.8,110.4,105.9$, 42.9, 37.8.

HRMS (ESI ${ }^{+}$): Calcd for $\mathrm{C}_{13} \mathrm{H}_{11} \mathrm{O}_{2}[\mathrm{M}+\mathrm{H}]^{+}:$199.0754. Found: 199.0755 .<smiles>COc1ccccc1C1CC(=O)c2cc(F)ccc21</smiles>

\section{(R)-6-Fluoro-3-(2-methoxyphenyl)-1-indanone (4m)}

The product was isolated by flash chromatography (ethyl acetate/hexanes 1:15) as yellow solid. $32.5 \mathrm{mg}$, 63\% yield. $97 \%$ ee.

$[\alpha]^{23}{ }_{\mathrm{D}}=+46.0^{\circ}\left(c=0.85, \mathrm{CHCl}_{3}\right)$.

HPLC: Daicel Chiralcel IC-3, $n$-hexane/isopropanol 95/5, flow rate $=1.0 \mathrm{~mL} / \mathrm{min}$. 
mAU

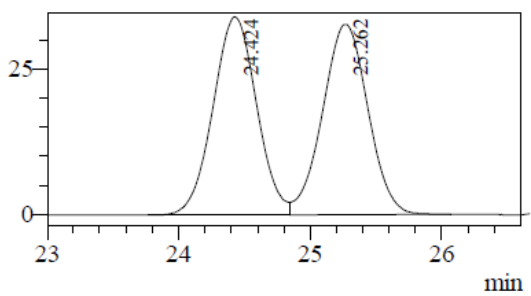

1 PDA Multi $1 / 220 \mathrm{~nm} 4 \mathrm{~nm}$

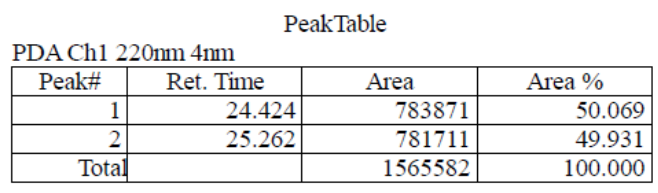

mAU

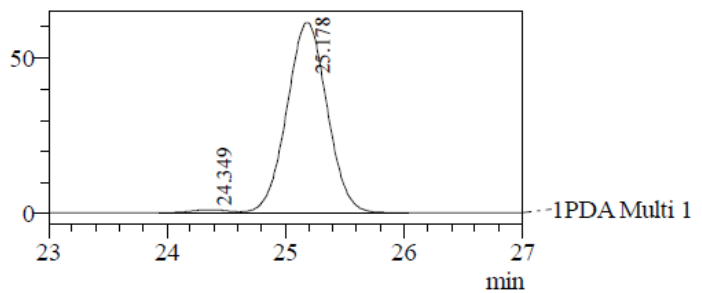

1 PDA Multi $1 / 220 \mathrm{~nm} 4 \mathrm{~nm}$

PDA Ch1 $220 \mathrm{~nm} 4 \mathrm{~nm}$
\begin{tabular}{|r|r|r|r|}
\hline Peak\# & Ret. Time & Area & \multicolumn{1}{|c|}{ Area $\%$} \\
\hline 1 & 24.349 & 23017 & 1.567 \\
\hline 2 & 25.178 & 1445816 & 98.433 \\
\hline Total & & 1468833 & 100.000 \\
\hline
\end{tabular}

${ }^{1} \mathrm{H}$ NMR $\left(400 \mathrm{MHz}, \mathrm{CDCl}_{3}\right): \delta 7.44(\mathrm{~d}, J=7.3 \mathrm{~Hz}, 1 \mathrm{H}), 7.31-7.23(\mathrm{~m}, 3 \mathrm{H}), 7.01-6.97(\mathrm{~m}, 1 \mathrm{H}), 6.93-6.88(\mathrm{~m}$, 2H), $4.86(\mathrm{~d}, J=5.3 \mathrm{~Hz}, 1 \mathrm{H}), 3.76(\mathrm{~s}, 3 \mathrm{H}), 3.23(\mathrm{dd}, J=19.2,8.1 \mathrm{~Hz}, 1 \mathrm{H}), 2.77$ (dd, $J=19.2,3.4 \mathrm{~Hz}, 1 \mathrm{H})$.

${ }^{13} \mathrm{C} \mathrm{NMR}\left(101 \mathrm{MHz}, \mathrm{CDCl}_{3}\right): \delta 205.6\left(\mathrm{~d}, J_{C-F}=2.9 \mathrm{~Hz}\right), 162.6\left(\mathrm{~d}, J_{C-F}=238.0 \mathrm{~Hz}\right), 157.4,153.4\left(\mathrm{~d}, J_{C-F}=1.7\right.$ $\mathrm{Hz}), 139.0$ (d, $\left.J_{C-F}=7.2 \mathrm{~Hz}\right), 131.3,128.51,128.46,128.2\left(\mathrm{~d}, J_{C-F}=6.9 \mathrm{~Hz}\right), 122.2\left(\mathrm{~d}, J_{C-F}=23.9 \mathrm{~Hz}\right), 120.9$, $111.1,109.3\left(\mathrm{~d}, J_{C-F}=11.7 \mathrm{~Hz}\right), 55.5,45.9,39.1$.

${ }^{19} \mathrm{~F}$ NMR (282 MHz, $\left.\mathrm{CDCl}_{3}\right): \delta-114.5$.

HRMS (ESI ${ }^{+}$): Calcd for $\mathrm{C}_{16} \mathrm{H}_{14} \mathrm{FO}_{2}[\mathrm{M}+\mathrm{H}]^{+}:$257.0972. Found: 257.0974 .<smiles>COc1ccccc1C1CC(=O)c2cc(Cl)ccc21</smiles>

\section{(R)-6-Chloro-3-(2-methoxyphenyl)-1-indanone (4n)}

The product was isolated by flash chromatography (ethyl acetate/hexanes 1:20) as yellow solid. $33.5 \mathrm{mg}, 62 \%$ yield. $89 \%$ ee.

$[\alpha]^{23}{ }_{\mathrm{D}}=+30.8^{\circ}\left(c=0.58, \mathrm{CHCl}_{3}\right)$.

HPLC: Daicel Chiralcel OJ-H, $n$-hexane/isopropanol 95/5, flow rate $=1.0 \mathrm{~mL} / \mathrm{min}$.

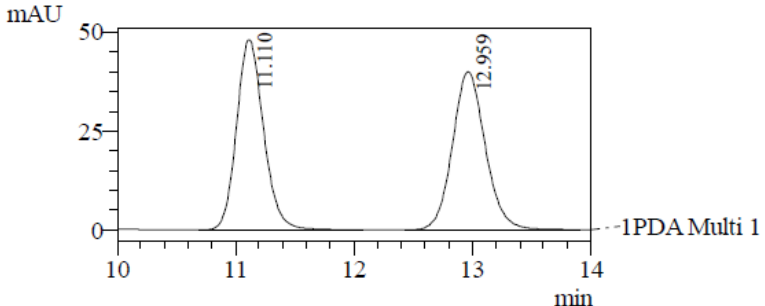

1 PDA Multi $1 / 220 \mathrm{~nm} 4 \mathrm{~nm}$

PeakTable

PDA Ch1 $220 \mathrm{~nm} 4 \mathrm{~nm}$
\begin{tabular}{|r|r|r|r|}
\hline Peak\# & Ret. Time & Area & \multicolumn{1}{c|}{ Area $\%$} \\
\hline 1 & 11.110 & 764333 & 49.742 \\
\hline 2 & 12.959 & 772253 & 50.258 \\
\hline Total & & 1536586 & 100.000 \\
\hline
\end{tabular}

mAU

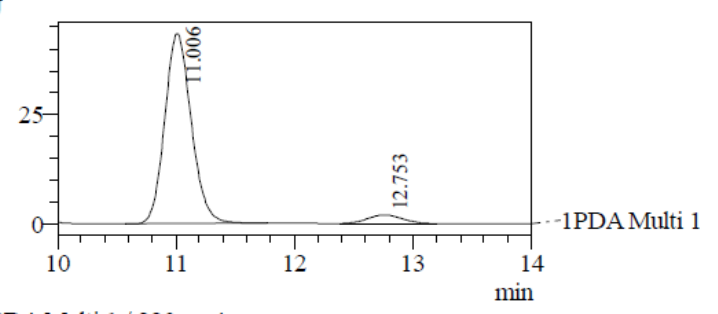

1 PDA Multi $1 / 220 \mathrm{~nm} 4 \mathrm{~nm}$

PeakTable

PDA Ch1 $220 \mathrm{~nm} 4 \mathrm{~nm}$
\begin{tabular}{|r|r|r|r|}
\hline Peak\# & Ret. Time & \multicolumn{1}{|c|}{ Area } & Area $\%$ \\
\hline 1 & 11.006 & 679197 & 94.509 \\
\hline 2 & 12.753 & 39464 & 5.491 \\
\hline Total & & 718661 & 100.000 \\
\hline
\end{tabular}


${ }^{1} \mathrm{H}$ NMR (400 MHz, $\left.\mathrm{CDCl}_{3}\right): \delta 7.75(\mathrm{~d}, J=2.0 \mathrm{~Hz}, 1 \mathrm{H}), 7.49(\mathrm{dd}, J=8.2,2.1 \mathrm{~Hz}, 1 \mathrm{H}), 7.27-7.22(\mathrm{~m}, 2 \mathrm{H}), 6.98-$ $6.96(\mathrm{~m}, 1 \mathrm{H}), 6.92-6.85(\mathrm{~m}, 2 \mathrm{H}), 4.82(\mathrm{dd}, J=8.0,3.4 \mathrm{~Hz}, 1 \mathrm{H}), 3.73(\mathrm{~s}, 3 \mathrm{H}), 3.18(\mathrm{dd}, J=19.2,8.0 \mathrm{~Hz}, 1 \mathrm{H})$, $2.74(\mathrm{dd}, J=19.2,3.6 \mathrm{~Hz}, 1 \mathrm{H})$.

${ }^{13} \mathrm{C}$ NMR (101 MHz, $\left.\mathrm{CDCl}_{3}\right): \delta 205.3,157.4,156.1,138.7,134.8,134.0,131.1,128.62,128.55,128.0,123.2$, $120.9,111.2,55.5,45.5,39.3$.

HRMS (ESI ${ }^{+}$): Calcd for $\mathrm{C}_{16} \mathrm{H}_{14} \mathrm{ClO}_{2}[\mathrm{M}+\mathrm{H}]^{+}: 273.0677$. Found: 273.0674 .<smiles>COc1ccc2c(c1)C(c1ccccc1OC)CC2=O</smiles>

\section{(R)-5-Methoxy-3-(2-methoxyphenyl)-1-indanone (40)}

The product was isolated by flash chromatography (ethyl acetate/hexanes 1:15) as yellow solid. $33.8 \mathrm{mg}, 63 \%$ yield. $98 \%$ ee.

$[\alpha]^{23} \mathrm{D}=+13.6^{\circ}\left(c=0.95, \mathrm{CHCl}_{3}\right)$.

HPLC: Daicel Chiralcel IC-3, $n$-hexane/isopropanol 95/5, flow rate $=1.0 \mathrm{~mL} / \mathrm{min}$.

maU

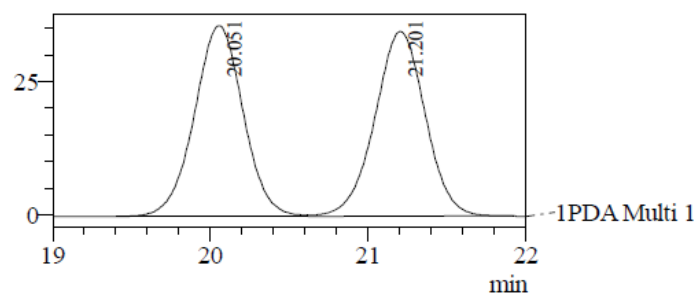

1 PDA Multi $1 / 220 \mathrm{~nm} 4 \mathrm{~nm}$

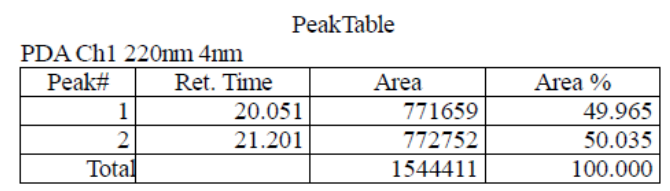

mAU

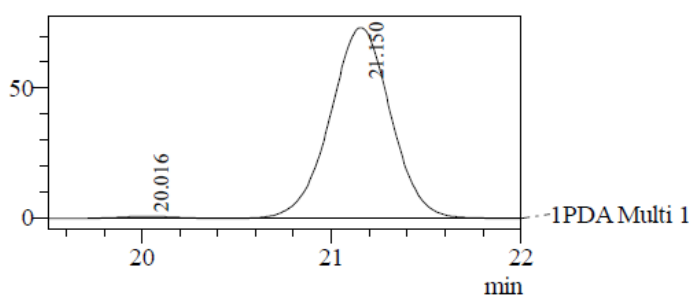

1 PDA Multi $1 / 220 \mathrm{~nm} 4 \mathrm{~nm}$

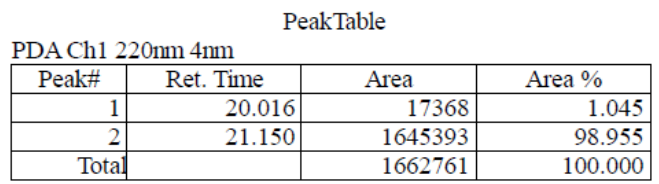

${ }^{1} \mathrm{H}$ NMR (400 MHz, $\left.\mathrm{CDCl}_{3}\right): \delta$ 7.28-7.12 (m, 4H), $6.93(\mathrm{dd}, J=7.4,1.6 \mathrm{~Hz}, 1 \mathrm{H}), 6.90-6.83(\mathrm{~m}, 2 \mathrm{H}), 4.84(\mathrm{dd}, J$ $=7.8,3.2 \mathrm{~Hz}, 1 \mathrm{H}), 3.86(\mathrm{~s}, 3 \mathrm{H}), 3.77(\mathrm{~s}, 3 \mathrm{H}), 3.20(\mathrm{dd}, J=19.2,7.9 \mathrm{~Hz}, 1 \mathrm{H}), 2.68(\mathrm{dd}, J=19.2,3.3 \mathrm{~Hz}, 1 \mathrm{H})$.

${ }^{13} \mathrm{C} \mathrm{NMR}\left(101 \mathrm{MHz}, \mathrm{CDCl}_{3}\right): \delta 206.8,159.7,157.4,150.7,138.5,132.1,128.3,128.2,127.6,124.2,120.8$, 111.0, 104.6, 55.8, 55.5, 46.1, 38.4 .

HRMS (ESI ${ }^{+}$): Calcd for $\mathrm{C}_{17} \mathrm{H}_{17} \mathrm{O}_{3}[\mathrm{M}+\mathrm{H}]^{+}:$269.1172. Found: 269.1166 . 
<smiles>Cc1ccc2c(c1)C(c1ccccc1)CC2=O</smiles>

\section{(S)-5-Methyl-3-phenyl-1-indanone (4p) [349547-17-7]}

The product was isolated by flash chromatography (ethyl acetate/hexanes 1:15) as yellow solid. $35.6 \mathrm{mg}, 80 \%$ yield. $97 \%$ ee.

$[\alpha]^{23}{ }_{\mathrm{D}}=+28.9^{\circ}\left(c=1.0, \mathrm{CHCl}_{3}\right)$.

HPLC: Daicel Chiralcel IC-3, $n$-hexane/isopropanol 95/5, flow rate $=1.0 \mathrm{~mL} / \mathrm{min}$.

mAU

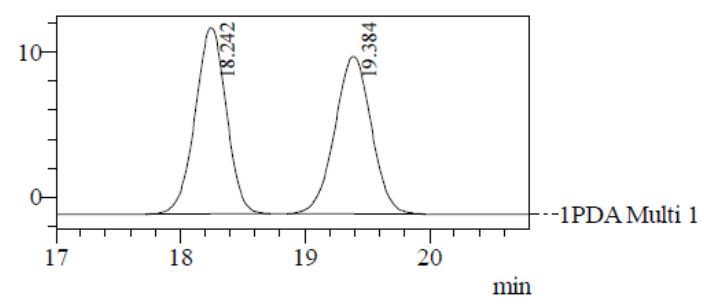

1 PDAMulti $1 / 220 \mathrm{~nm} 4 \mathrm{~nm}$

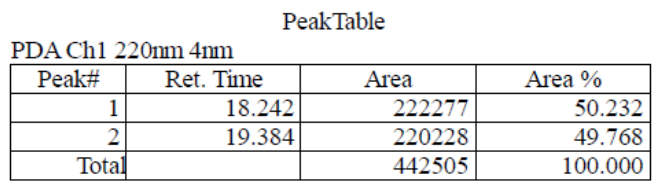

$\mathrm{mAU}$

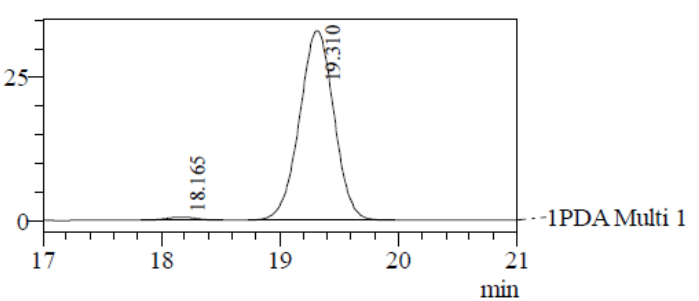

1 PDA Multi $1 / 220 \mathrm{~nm} 4 \mathrm{~nm}$

PDA Ch1 220nm 4nm
\begin{tabular}{|r|r|r|r|}
\hline \multicolumn{1}{|c|}{ Peak\# } & Ret. Time & \multicolumn{1}{|c|}{ Area } & \multicolumn{1}{c|}{ Area $\%$} \\
\hline 1 & 18.165 & 9875 & 1.460 \\
\hline 2 & 19.310 & 666499 & 98.540 \\
\hline Total & & 676374 & 100.000 \\
\hline
\end{tabular}

${ }^{1} \mathrm{H}$ NMR (400 MHz, $\left.\mathrm{CDCl}_{3}\right): \delta 7.70$ (d, $\left.J=7.9 \mathrm{~Hz}, 1 \mathrm{H}\right), 7.34-7.29(\mathrm{~m}, 2 \mathrm{H}), 7.28-7.20$ (m, 2H), 7.15-7.11 (m, 2H), $7.05(\mathrm{~s}, 1 \mathrm{H}), 4.52(\mathrm{dd}, J=8.0,3.7 \mathrm{~Hz}, 1 \mathrm{H}), 3.21(\mathrm{dd}, J=19.1,8.0 \mathrm{~Hz}, 1 \mathrm{H}), 2.68(\mathrm{dd}, J=19.1,3.8 \mathrm{~Hz}$, $1 \mathrm{H}), 2.37(\mathrm{~s}, 4 \mathrm{H})$.

${ }^{13} \mathrm{C} \mathrm{NMR}\left(101 \mathrm{MHz}, \mathrm{CDCl}_{3}\right): \delta 205.6,158.6,146.5,144.0,134.7,129.3,129.0,127.8,127.2,127.1,123.4$, 47.2, 44.5, 22.2.

MS (ESI $\left.{ }^{+}\right)$: Calcd for $\mathrm{C}_{16} \mathrm{H}_{15} \mathrm{O}[\mathrm{M}+\mathrm{H}]^{+}:$223.11. Found: 223.22.<smiles>COc1ccc(C2CC(=O)c3cc(OC)cc(OC)c32)cc1</smiles>

\section{(S)-4,6-Dimethoxy-3-(4-methoxyphenyl)-1-indanone (4q) [1983136-72-6]}

The product was isolated by flash chromatography (ethyl acetate/hexanes 1:15) as yellow solid. 35.8mg, 60\% yield. $96 \%$ ee.

$[\alpha]^{23}{ }_{\mathrm{D}}=+26.7^{\circ}\left(c=0.65, \mathrm{CHCl}_{3}\right)$. Literature value: $[\alpha]^{20}{ }_{\mathrm{D}}=+36^{\circ}\left(c=0.14, \mathrm{CHCl}_{3}\right)$ for a sample of $99 \%$ ee $(S)$. 
HPLC: Daicel Chiralcel AD-H, $n$-hexane/isopropanol 90/10, flow rate $=1.0 \mathrm{~mL} / \mathrm{min}$.

$\mathrm{mAU}$

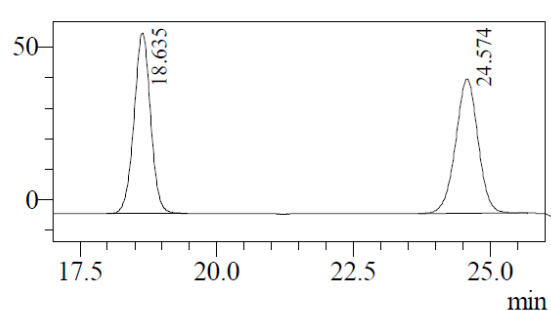

1 PDAMulti $1 / 220 \mathrm{~nm} 4 \mathrm{~nm}$

PDA Ch1 $220 \mathrm{~nm}$ 4nm
\begin{tabular}{|r|r|r|r|}
\hline Peak $\#$ & Ret. Time & Area & Area $\%$ \\
\hline 1 & 18.635 & 1248971 & 49.947 \\
\hline 2 & 24.574 & 1251625 & 50.053 \\
\hline Total & & 2500596 & 100.000 \\
\hline
\end{tabular}

mAU

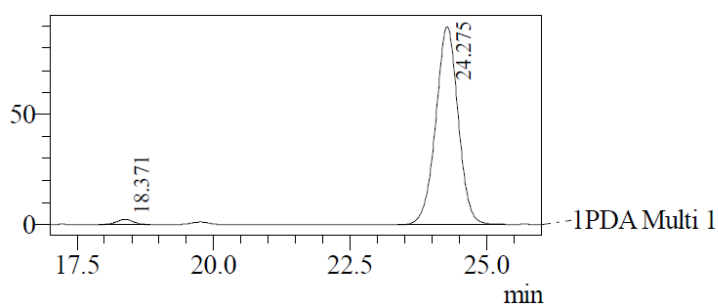

1 PDA Multi $1 / 220 \mathrm{~nm} 4 \mathrm{~nm}$

PDA Ch1 $220 \mathrm{~nm}$ 4nm
\begin{tabular}{|r|r|r|r|}
\hline Peak\# & Ret. Time & Area & Area $\%$ \\
\hline 1 & 18.371 & 49271 & 1.882 \\
\hline 2 & 24.275 & 2568678 & 98.118 \\
\hline Total & & 2617949 & 100.000 \\
\hline
\end{tabular}

${ }^{1} \mathrm{H}$ NMR $\left(400 \mathrm{MHz}, \mathrm{CDCl}_{3}\right): \delta 6.99-6.94(\mathrm{~m}, 2 \mathrm{H}), 6.84(\mathrm{~d}, J=2.1 \mathrm{~Hz}, 1 \mathrm{H}), 6.81-6.75(\mathrm{~m}, 2 \mathrm{H}), 6.63(\mathrm{~d}, J=2.1$ $\mathrm{Hz}, 1 \mathrm{H}), 4.53(\mathrm{dd}, J=7.8,2.1 \mathrm{~Hz}, 1 \mathrm{H}), 3.86(\mathrm{~s}, 3 \mathrm{H}), 3.77$ (s, 3H), 3.66 (s, 3H), 3.19 (dd, J = 19.2, 7.9 Hz, 1H), $2.58(\mathrm{dd}, J=19.2,2.2 \mathrm{~Hz}, 1 \mathrm{H})$.

${ }^{13} \mathrm{C} \mathrm{NMR}\left(101 \mathrm{MHz}, \mathrm{CDCl}_{3}\right): \delta 206.7,161.8,158.2,158.0,139.9,139.1,136.2,128.1,114.0,106.3,96.0,55.9$, $55.8,55.4,47.9,40.6$.

MS (ESI): Calcd for $\mathrm{C}_{18} \mathrm{H}_{19} \mathrm{O}_{4}[\mathrm{M}+\mathrm{H}]^{+}: 299.13$. Found: 299.15 .<smiles>COc1ccccc1C1c2ccccc2C(=O)C1C</smiles>

\section{(2R,3R)-2-Methyl-3-(2-methoxyphenyl)-1-indanone (4r)}

The product was isolated by flash chromatography (ethyl acetate/hexanes 1:15) as yellow solid. $30.7 \mathrm{mg}, 61 \%$ yield. $86 \%$ ee. The trans configuration on the indane was assigned based on magnetization transfer between $\alpha$ hydrogen and benzylic hydrogen in NOESY.

$[\alpha]^{23}{ }_{\mathrm{D}}=+1.0^{\circ}\left(c=0.45, \mathrm{CHCl}_{3}\right)$.

HPLC: Daicel Chiralcel IC-3, $n$-hexane/isopropanol 95/5, flow rate $=0.5 \mathrm{~mL} / \mathrm{min}$. 
mAU

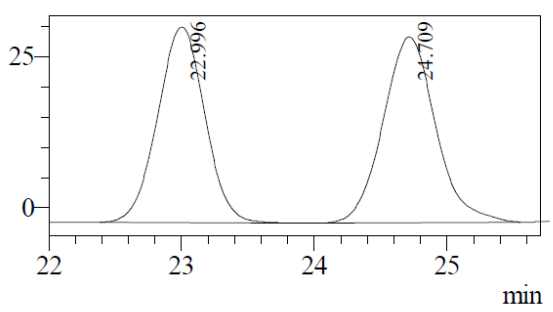

1 PDA Multi $1 / 220 \mathrm{~nm} 4 \mathrm{~nm}$

PDA Ch1 220nm 4nm
\begin{tabular}{|r|r|r|r|}
\hline Peak\# & Ret. Time & Area & \multicolumn{1}{|c|}{ Area \% } \\
\hline 1 & 22.996 & 796079 & 48.495 \\
\hline 2 & 24.709 & 845492 & 51.505 \\
\hline Total & & 1641571 & 100.000 \\
\hline
\end{tabular}

mAU

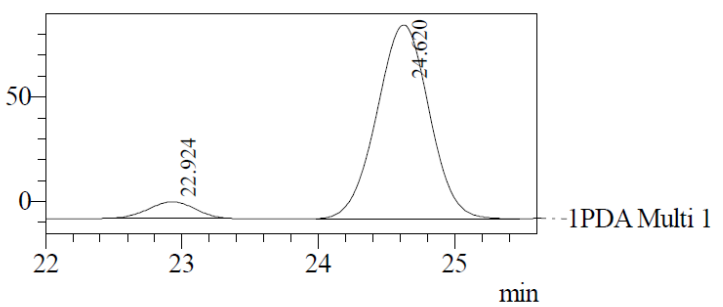

PDA Multi 1 / 220nm 4nm

PeakTable
\begin{tabular}{|r|c|r|r|}
\hline \multicolumn{1}{|c|}{ Peak\# $\#$ ( } & Ret. Time & Area & Area $\%$ \\
\hline 1 & 22.924 & 183274 & 6.899 \\
\hline 2 & 24.620 & 2473085 & 93.101 \\
\hline Total & & 2656359 & 100.000 \\
\hline
\end{tabular}

${ }^{1} \mathrm{H}$ NMR (400 MHz, $\left.\mathrm{CDCl}_{3}\right): \delta 7.80(\mathrm{~d}, J=7.6 \mathrm{~Hz}, 1 \mathrm{H}), 7.54(\psi \mathrm{t}, J=7.5 \mathrm{~Hz}, 1 \mathrm{H}), 7.39(\psi \mathrm{t}, J=7.5 \mathrm{~Hz}, 1 \mathrm{H})$, 7.28- $7.20(\mathrm{~m}, 2 \mathrm{H}), 6.96(\mathrm{~d}, J=6.6 \mathrm{~Hz}, 1 \mathrm{H}), 6.93-6.87(\mathrm{~m}, 2 \mathrm{H}), 4.44(\mathrm{~d}, J=3.1 \mathrm{~Hz}, 1 \mathrm{H}), 3.75(\mathrm{~s}, 3 \mathrm{H}), 2.78-2.68$ (m, 1H), $1.38(\mathrm{~d}, J=7.4 \mathrm{~Hz}, 3 \mathrm{H})$.

${ }^{13} \mathrm{C}$ NMR (101 MHz, $\left.\mathrm{CDCl}_{3}\right): \delta 209.0,157.7,156.72,136.4,134.9,131.3,129.1,128.3,127.6,126.4,123.7$, 121.0, 111.1, 55.5, 51.9, 48.2, 15.5 .

HRMS (ESI ${ }^{+}$): Calcd for $\mathrm{C}_{17} \mathrm{H}_{17} \mathrm{O}_{2}[\mathrm{M}+\mathrm{H}]^{+}:$253.1223. Found: 253.1225 .<smiles>O=C1CC(/C=C/c2ccccc2)c2ccccc21</smiles>

\section{$(S)-3-(E)-S t y r y l-1-i n d a n o n e ~(6 a)$}

The product was isolated by flash chromatography (ethyl acetate/hexanes 1:20) as yellow solid. $35.5 \mathrm{mg}, 76 \%$ yield. $97 \%$ ee.

$[\alpha]^{23} \mathrm{D}=+2.2^{\circ}\left(c=0.52, \mathrm{CHCl}_{3}\right)$.

HPLC: Daicel Chiralcel OD-H, $n$-hexane/isopropanol 95/5, flow rate $1.0 \mathrm{~mL} / \mathrm{min}$.

$\mathrm{mAU}$

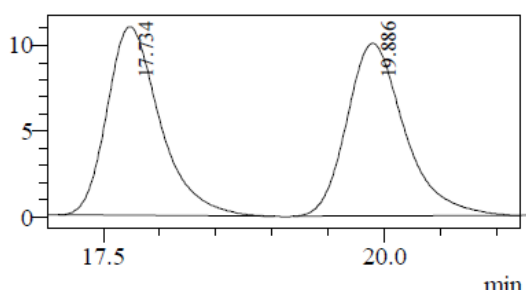

1 PDA Multi $1 / 220 \mathrm{~nm} 4 \mathrm{~nm}$

PDA Ch1 220nm 4nm
\begin{tabular}{|r|r|r|r|}
\hline Peak\# & Ret. Time & Area & Area $\%$ \\
\hline 1 & 17.734 & 360844 & 50.087 \\
\hline 2 & 19.886 & 359584 & 49.913 \\
\hline Total & & 720428 & 100.000 \\
\hline
\end{tabular}

mAU

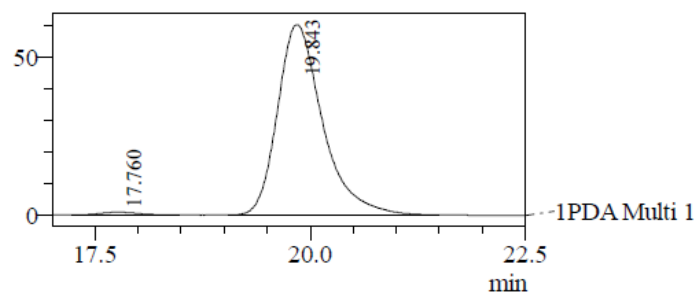

1 PDA Multi $1 / 220 \mathrm{~nm} 4 \mathrm{~nm}$

PDA Ch1 $220 \mathrm{~nm}$ 4nm
\begin{tabular}{|r|r|r|r|}
\hline Peak\# & Ret. Time & \multicolumn{1}{c|}{ Area } & Area $\%$ \\
\hline 1 & 17.760 & 28454 & 1.286 \\
\hline 2 & 19.843 & 2183880 & 98.714 \\
\hline Total & & 2212334 & 100.000 \\
\hline
\end{tabular}


${ }^{1} \mathrm{H}$ NMR (400 MHz, $\left.\mathrm{CDCl}_{3}\right): \delta 7.79(\mathrm{~d}, J=7.7 \mathrm{~Hz}, 1 \mathrm{H}), 7.65-7.60(\mathrm{~m}, 1 \mathrm{H}), 7.51(\mathrm{~d}, J=7.8 \mathrm{~Hz}, 1 \mathrm{H}), 7.43(\mathrm{t}, J=$ $7.4 \mathrm{~Hz}, 1 \mathrm{H}), 7.40-7.36(\mathrm{~m}, 2 \mathrm{H}), 7.32$ ( $\psi \mathrm{t}, J=7.5 \mathrm{~Hz}, 2 \mathrm{H}), 7.28-7.22(\mathrm{~m}, 1 \mathrm{H}), 6.64(\mathrm{~d}, J=15.7 \mathrm{~Hz}, 1 \mathrm{H}), 6.19$ $(\mathrm{dd}, J=15.7,8.9 \mathrm{~Hz}, 1 \mathrm{H}), 4.19-4.14(\mathrm{~m}, 1 \mathrm{H}), 3.08(\mathrm{dd}, J=19.1,7.8 \mathrm{~Hz}, 1 \mathrm{H}), 2.59$ (dd, $J=19.1,3.9 \mathrm{~Hz}, 1 \mathrm{H})$. ${ }^{13} \mathrm{C}$ NMR $\left(101 \mathrm{MHz}, \mathrm{CDCl}_{3}\right): \delta 205.6,156.8,136.9,136.7,135.0,131.7,131.1,128.8,128.5,127.8,126.8$, $126.5,123.8,44.1,42.4$.

HRMS (ESI ${ }^{+}$): Calcd for $\mathrm{C}_{17} \mathrm{H}_{15} \mathrm{O}[\mathrm{M}+\mathrm{H}]^{+}:$235.1117. Found: 235.1121 .

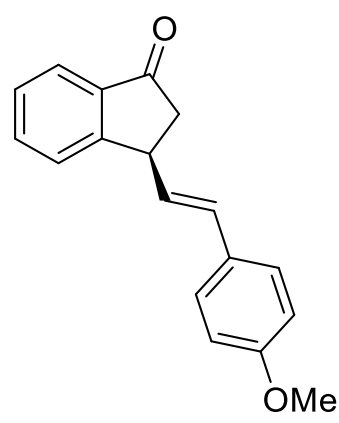

\section{(S)-3-(E)-(4-Methoxystyryl)-1-indanone (6b)}

The product was isolated by flash chromatography (ethyl acetate/hexanes 1:20) as yellow solid. $34.2 \mathrm{mg}, 65 \%$ yield. $90 \%$ ee.

$[\alpha]^{23} \mathrm{D}=+28.7^{\circ}\left(c=0.81, \mathrm{CHCl}_{3}\right)$.

HPLC: Daicel Chiralcel OZ-3, $n$-hexane/isopropanol 95/5, flow rate $1.0 \mathrm{~mL} / \mathrm{min}$.

$\mathrm{mAU}$

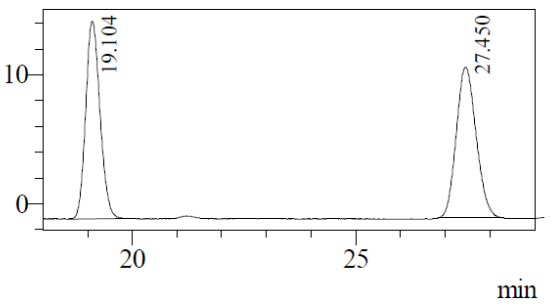

$1 \mathrm{~F}$

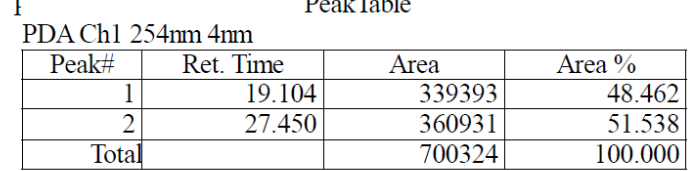

$\mathrm{mAU}$

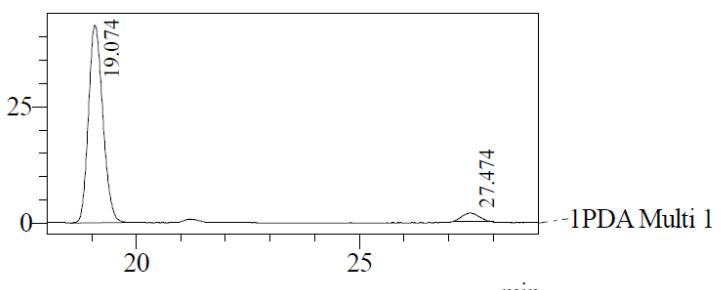

$1 \mathrm{I}$

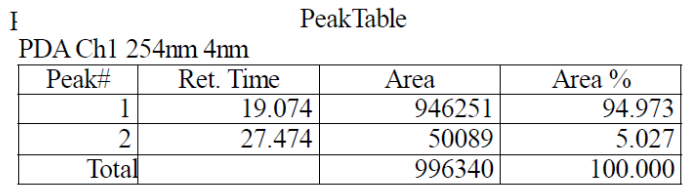

${ }^{1} \mathrm{H}$ NMR (400 MHz, $\left.\mathrm{CDCl}_{3}\right): \delta 7.78(\mathrm{~d}, J=7.6 \mathrm{~Hz}, 1 \mathrm{H}), 7.64-7.59(\mathrm{~m}, 1 \mathrm{H}), 7.51(\mathrm{~d}, J=7.7 \mathrm{~Hz}, 1 \mathrm{H}), 7.42(\psi \mathrm{t}, J$ $=7.4 \mathrm{~Hz}, 1 \mathrm{H}), 7.31(\mathrm{~d}, J=8.7 \mathrm{~Hz}, 2 \mathrm{H}), 6.87-6.84(\mathrm{~m}, 2 \mathrm{H}), 6.58(\mathrm{~d}, J=15.6 \mathrm{~Hz}, 1 \mathrm{H}), 6.04(\mathrm{dd}, J=15.6,8.9 \mathrm{~Hz}$, $1 \mathrm{H}), 4.13(\mathrm{td}, J=8.3,3.9 \mathrm{~Hz}, 1 \mathrm{H}), 3.81(\mathrm{~s}, 3 \mathrm{H}), 3.06(\mathrm{dd}, J=19.1,7.7 \mathrm{~Hz}, 1 \mathrm{H}), 2.57$ (dd, $J=19.1,3.9 \mathrm{~Hz}, 1 \mathrm{H})$. ${ }^{13} \mathrm{C}$ NMR $\left(101 \mathrm{MHz}, \mathrm{CDCl}_{3}\right): \delta 205.8,159.4,157.1,136.7,135.0,131.0,129.6,128.9,128.1,127.6,126.8$, 123.7, 114.2, 55.5, 44.2, 42.4.

HRMS (ESI ${ }^{+}$): Calcd for $\mathrm{C}_{18} \mathrm{H}_{17} \mathrm{O}_{2}[\mathrm{M}+\mathrm{H}]^{+}:$265.1223. Found: 265.1226. 
<smiles>O=C1C[C@H](/C=C/c2ccc(F)cc2)c2ccccc21</smiles>

\section{(S)-3-(E)-(4-Fluorostyryl)-1-indanone (6c)}

The product was isolated by flash chromatography (ethyl acetate/hexanes 1:20) as yellow solid. $35.2 \mathrm{mg}, 70 \%$ yield. 94\% ee.

$[\alpha]^{23} \mathrm{D}=+16.5^{\circ}\left(c=0.62, \mathrm{CHCl}_{3}\right)$.

HPLC: Daicel Chiralcel OZ-3, $n$-hexane/isopropanol 95/5, flow rate $1.0 \mathrm{~mL} / \mathrm{min}$.
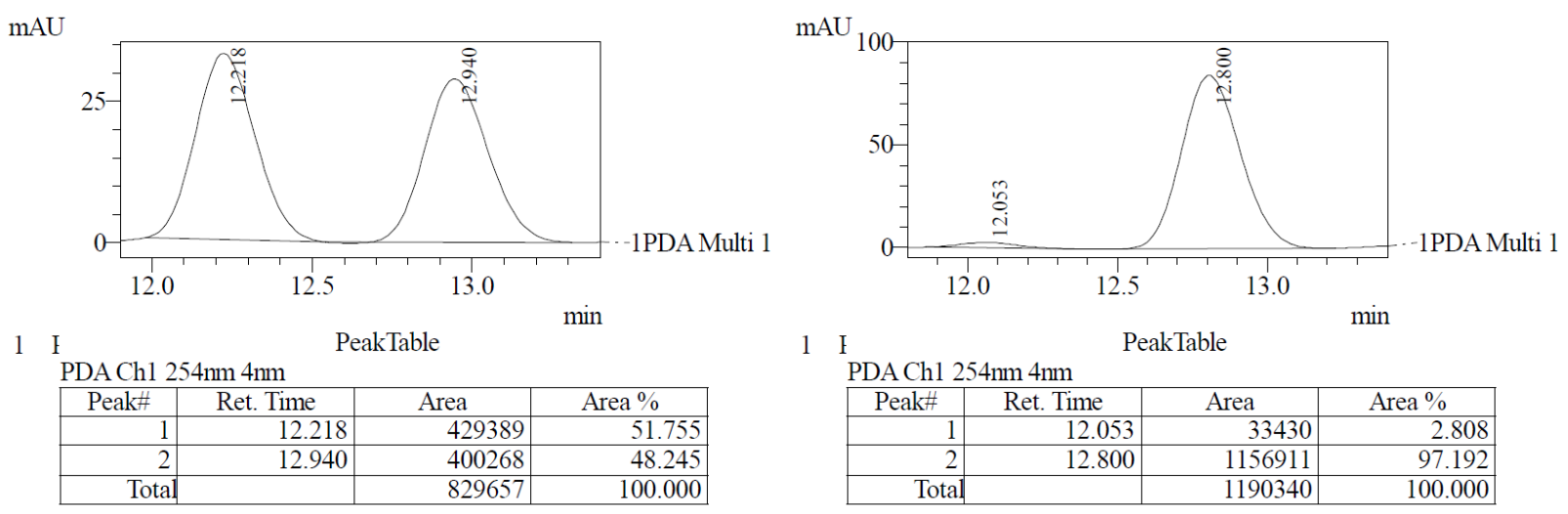

${ }^{1} \mathrm{H}$ NMR (400 MHz, $\left.\mathrm{CDCl}_{3}\right): \delta 7.79(\mathrm{~d}, J=7.7 \mathrm{~Hz}, 1 \mathrm{H}), 7.63(\psi \mathrm{td}, J=7.6,1.2 \mathrm{~Hz}, 1 \mathrm{H}), 7.50(\mathrm{dd}, J=7.7,0.8$ $\mathrm{Hz}, 1 \mathrm{H}), 7.43$ ( $\psi \mathrm{t}, J=7.4 \mathrm{~Hz}, 1 \mathrm{H}), 7.37-7.31(\mathrm{~m}, 2 \mathrm{H}), 7.03-6.98(\mathrm{~m}, 2 \mathrm{H}), 6.60(\mathrm{~d}, J=15.7 \mathrm{~Hz}, 1 \mathrm{H}), 6.10(\mathrm{dd}, J$ $=15.7,8.9 \mathrm{~Hz}, 1 \mathrm{H}), 4.17-4.12(\mathrm{~m}, 1 \mathrm{H}), 3.07(\mathrm{dd}, J=19.1,7.8 \mathrm{~Hz}, 1 \mathrm{H}), 2.58(\mathrm{dd}, J=19.1,3.9 \mathrm{~Hz}, 1 \mathrm{H})$.

${ }^{13} \mathrm{C}$ NMR (101 MHz, $\left.\mathrm{CDCl}_{3}\right): \delta 205.5,162.5\left(\mathrm{~d}, J_{C-F}=247.0 \mathrm{~Hz}\right), 156.7,136.7,135.1,133.0\left(\mathrm{~d}, J_{C-F}=3.2 \mathrm{~Hz}\right)$, $130.9\left(\mathrm{~d}, J_{C-F}=2.1 \mathrm{~Hz}\right), 130.5,128.2\left(\mathrm{~d}, J_{C-F}=9.3 \mathrm{~Hz}\right), 127.9,126.8,123.8,115.8,115.6\left(\mathrm{~d}, J_{C-F}=21.6 \mathrm{~Hz}\right)$, $44.1,42.3$.

${ }^{19} \mathrm{~F}$ NMR (377 MHz, $\left.\mathrm{CDCl}_{3}\right): \delta-114.3$.

HRMS (ESI ${ }^{+}$): Calcd for $\mathrm{C}_{17} \mathrm{H}_{14} \mathrm{FO}[\mathrm{M}+\mathrm{H}]^{+}: 253.1023$. Found: 253.1027.<smiles>COc1ccccc1/C=C/C1CC(=O)c2ccccc21</smiles> 
The product was isolated by flash chromatography (ethyl acetate/hexanes 1:20) as yellow solid. $33.2 \mathrm{mg}, 63 \%$ yield. $97 \%$ ee.

$[\alpha]^{23}{ }_{\mathrm{D}}=+20.1^{\circ}\left(c=1.21, \mathrm{CHCl}_{3}\right)$.

HPLC: Daicel Chiralcel OZ-3, $n$-hexane/isopropanol 95/5, flow rate $1.0 \mathrm{~mL} / \mathrm{min}$.

$\mathrm{mAU}$

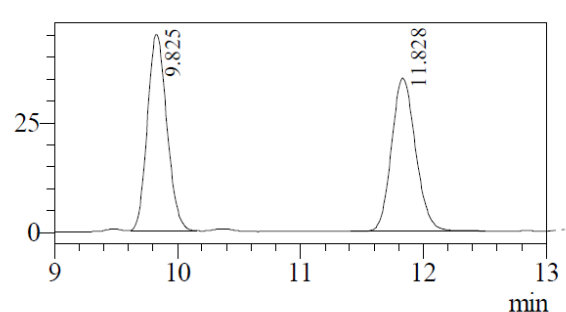

$1 \mathrm{I}$

I PeakTable
PDA Ch1 $254 \mathrm{~nm} 4 \mathrm{~nm}$
\begin{tabular}{|r|r|r|r|}
\hline Peak\# & Ret. Time & Area & Area $\%$ \\
\hline 1 & 9.825 & 490962 & 50.793 \\
\hline 2 & 11.828 & 475635 & 49.207 \\
\hline Total & & 966597 & 100.000 \\
\hline
\end{tabular}

$\mathrm{mAU}$

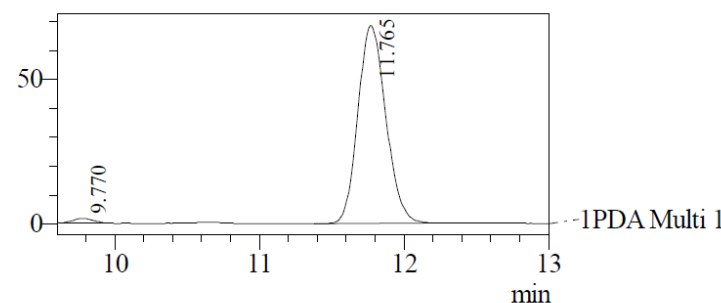

$1 \mathrm{I}$

PDA Ch1 $254 \mathrm{~nm} 4 \mathrm{~nm}$
\begin{tabular}{|r|r|r|r|}
\hline Peak\# & Ret. Time & \multicolumn{1}{|c|}{ Area } & Area $\%$ \\
\hline 1 & 9.770 & 15566 & 1.663 \\
\hline 2 & 11.765 & 920653 & 98.337 \\
\hline Total & & 936218 & 100.000 \\
\hline
\end{tabular}

${ }^{1} \mathrm{H}$ NMR $\left(400 \mathrm{MHz}, \mathrm{CDCl}_{3}\right): \delta 7.78(\mathrm{~d}, J=7.7 \mathrm{~Hz}, 1 \mathrm{H}), 7.63-7.61(\mathrm{~m}, 1 \mathrm{H}), 7.53-7.50(\mathrm{~m}, 1 \mathrm{H}), 7.42-7.39(\mathrm{~m}$, 2H), 7.25- 7.21(m, 1H), $6.98(\mathrm{~d}, J=15.6 \mathrm{~Hz}, 1 \mathrm{H}), 6.94-6.86(\mathrm{~m}, 2 \mathrm{H}), 6.17(\mathrm{dd}, J=15.7,7.8 \mathrm{~Hz}, 1 \mathrm{H}), 4.19-4.14$ (m, 1H), $3.86(\mathrm{~s}, 3 \mathrm{H}), 3.08(\mathrm{dd}, J=13.8,6.9 \mathrm{~Hz}, 1 \mathrm{H}), 2.60(\mathrm{dd}, J=15.2,4.0 \mathrm{~Hz}, 1 \mathrm{H})$.

${ }^{13} \mathrm{C}$ NMR $\left(101 \mathrm{MHz}, \mathrm{CDCl}_{3}\right): \delta 205.9,157.2,156.7,136.6,135.0,131.6,128.8,128.0,126.9,126.8,126.4$, $125.8,123.7,120.8,111.0,55.6,44.1,42.9$.

HRMS (ESI ${ }^{+}$: Calcd for $\mathrm{C}_{18} \mathrm{H}_{17} \mathrm{O}_{2}[\mathrm{M}+\mathrm{H}]^{+}:$265.1223. Found: 265.1235.<smiles>O=C1C[C@H](/C=C/c2ccco2)c2ccccc21</smiles>

\section{(S)-3-(E)-(2-(Furan-2-yl)vinyl)-1-indanone (6e)}

The product was isolated by flash chromatography (ethyl acetate/hexanes 1:20) as yellow oil. $26.9 \mathrm{mg}, 60 \%$ yield. $94 \%$ ee.

$[\alpha]^{23} \mathrm{D}=+16.5^{\circ}\left(c=0.65, \mathrm{CHCl}_{3}\right)$.

HPLC: Daicel Chiralcel OZ-3, $n$-hexane/isopropanol 95/5, flow rate $1.0 \mathrm{~mL} / \mathrm{min}$. 
$\mathrm{mAU}$

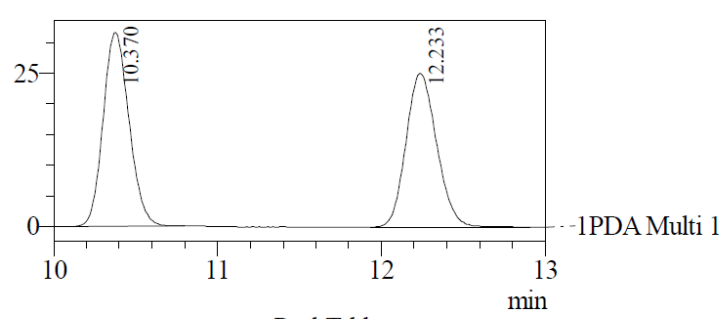

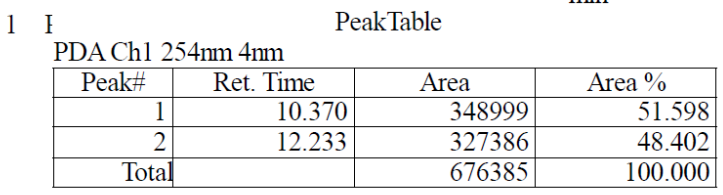

$\mathrm{mAU}$

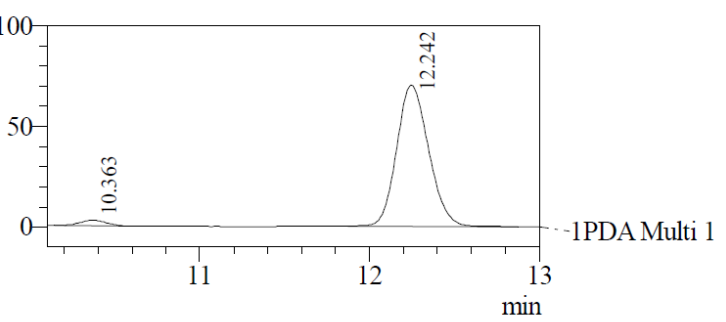

$1 \mathrm{I}$

PDACh1 $254 \mathrm{~nm} 4 \mathrm{~nm}$
\begin{tabular}{|r|r|r|r|}
\hline Peak\# & Ret. Time & \multicolumn{1}{|c|}{ Area } & Area $\%$ \\
\hline 1 & 10.363 & 31642 & 3.298 \\
\hline 2 & 12.242 & 927709 & 96.702 \\
\hline Total & & 959351 & 100.000 \\
\hline
\end{tabular}

${ }^{1} \mathrm{H}$ NMR $\left(400 \mathrm{MHz}, \mathrm{CDCl}_{3}\right): \delta 7.78(\mathrm{~d}, J=7.7 \mathrm{~Hz}, 1 \mathrm{H}), 7.64-7.60(\mathrm{~m}, 1 \mathrm{H}), 7.52-7.50(\mathrm{~m}, 1 \mathrm{H}), 7.44-7.39(\mathrm{~m}$, 1H), 7.33-7.32(m, 1H), $6.43(\mathrm{~d}, J=15.7 \mathrm{~Hz}, 1 \mathrm{H}), 6.37$ (dd, $J=3.3,1.8 \mathrm{~Hz}, 1 \mathrm{H}), 6.24(\mathrm{~d}, J=3.3 \mathrm{~Hz}, 1 \mathrm{H}), 6.16$ (dd, $J=15.7,8.9 \mathrm{~Hz}, 1 \mathrm{H}), 4.14-4.09(\mathrm{~m}, 1 \mathrm{H}), 3.05(\mathrm{dd}, J=18.8,7.8 \mathrm{~Hz}, 1 \mathrm{H}), 2.57(\mathrm{dd}, J=19.2,3.9 \mathrm{~Hz}, 1 \mathrm{H})$. ${ }^{13} \mathrm{C} \mathrm{NMR}\left(101 \mathrm{MHz}, \mathrm{CDCl}_{3}\right): \delta 205.5,156.6,152.4,142.1,136.7,135.0,129.8,128.2,126.8,123.8,120.0$, 111.4, 107.9, 44.1, 42.1.

HRMS (ESI ${ }^{+}$): Calcd for $\mathrm{C}_{15} \mathrm{H}_{13} \mathrm{O}_{2}[\mathrm{M}+\mathrm{H}]^{+}:$225.0910. Found: 225.0923 .<smiles>C/C(=C\c1ccccc1)C1CC(=O)c2ccccc21</smiles>

\section{(S)-3-(E)-(1-Phenylprop-1-en-2-yl)-1-indanone (6f)}

The product was isolated by flash chromatography (ethyl acetate/hexanes 1:20) as yellow oil. $32.2 \mathrm{mg}, 65 \%$ yield. 94\% ee.

$[\alpha]^{23}{ }_{\mathrm{D}}=+25.1^{\circ}\left(c=1.08, \mathrm{CHCl}_{3}\right)$.

HPLC: Daicel Chiralcel OZ-3, $n$-hexane/isopropanol 95/5, flow rate $1.0 \mathrm{~mL} / \mathrm{min}$.

$\mathrm{mAU}$

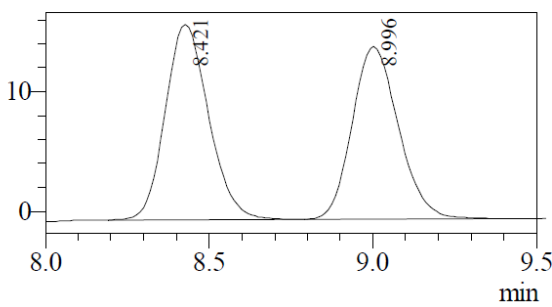

$1 \mathrm{I}$

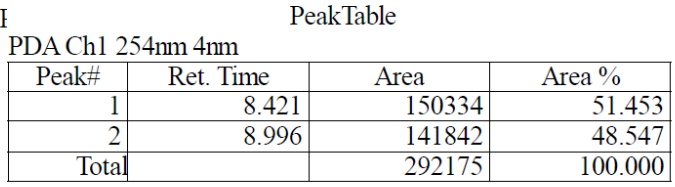

$\mathrm{mAU}$

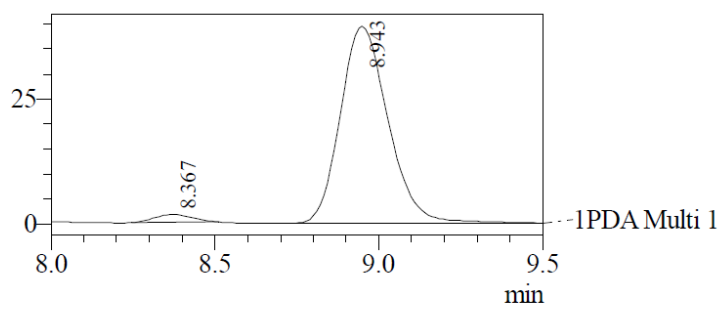

$1 \mathrm{H}$

PDA Ch1 $254 \mathrm{~nm}$ 4nm
\begin{tabular}{|r|r|r|r|}
\hline Peak\# & Ret. Time & \multicolumn{1}{|c|}{ Area } & Area $\%$ \\
\hline 1 & 8.367 & 12671 & 3.061 \\
\hline 2 & 8.943 & 401236 & 96.939 \\
\hline Total & & 413908 & 100.000 \\
\hline
\end{tabular}


${ }^{1} \mathrm{H}$ NMR (400 MHz, $\left.\mathrm{CDCl}_{3}\right): \delta 7.80(\mathrm{~d}, J=7.7 \mathrm{~Hz}, 1 \mathrm{H}), 7.63(\psi \mathrm{t}, J=7.4 \mathrm{~Hz}, 1 \mathrm{H}), 7.50(\mathrm{~d}, J=7.7 \mathrm{~Hz}, 1 \mathrm{H})$, $7.43(\psi \mathrm{t}, J=7.4 \mathrm{~Hz}, 1 \mathrm{H}), 7.38-7.31(\mathrm{~m}, 2 \mathrm{H}), 7.30-7.28(\mathrm{~m}, 2 \mathrm{H}), 7.23(\psi \mathrm{t}, J=7.1 \mathrm{~Hz}, 1 \mathrm{H}), 6.57(\mathrm{~s}, 1 \mathrm{H}), 4.21$ (dd, $J=7.9,3.5 \mathrm{~Hz}, 1 \mathrm{H}), 2.99(\mathrm{dd}, J=19.2,8.0 \mathrm{~Hz}, 1 \mathrm{H}), 2.63(\mathrm{dd}, J=19.2,3.6 \mathrm{~Hz}, 1 \mathrm{H}), 1.61(\mathrm{~s}, 3 \mathrm{H})$.

${ }^{13} \mathrm{C} \mathrm{NMR}\left(101 \mathrm{MHz}, \mathrm{CDCl}_{3}\right): \delta 206.0,156.8,138.3,137.7,137.5,135.0,129.1,128.3,128.2,128.1,126.7$, $126.5,123.7,48.7,42.5,14.2$.

HRMS (ESI ${ }^{+}$: Calcd for $\mathrm{C}_{18} \mathrm{H}_{17} \mathrm{O}[\mathrm{M}+\mathrm{H}]^{+}: 249.1274$. Found: 249.1269.

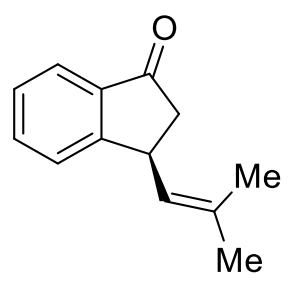

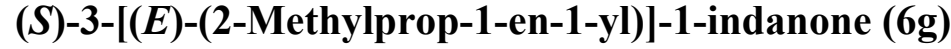

The product was isolated by flash chromatography (ethyl acetate/hexanes 1:20) as yellow oil. 26.2 mg, 70\% yield. $96 \%$ ee.

$[\alpha]^{23} \mathrm{D}=+2.2^{\circ}\left(c=0.52, \mathrm{CHCl}_{3}\right)$.

HPLC: Daicel Chiralcel OD-H, $n$-hexane/isopropanol 95/5, flow rate $0.5 \mathrm{~mL} / \mathrm{min}$.

$\mathrm{mAU}$

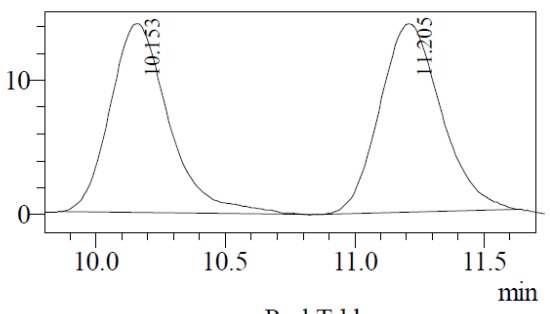

I

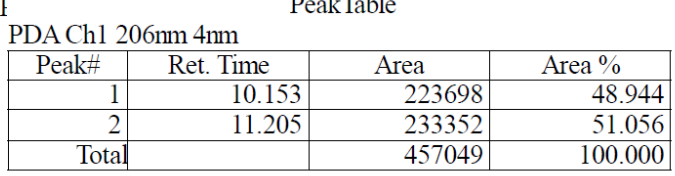

$\mathrm{mAU}$

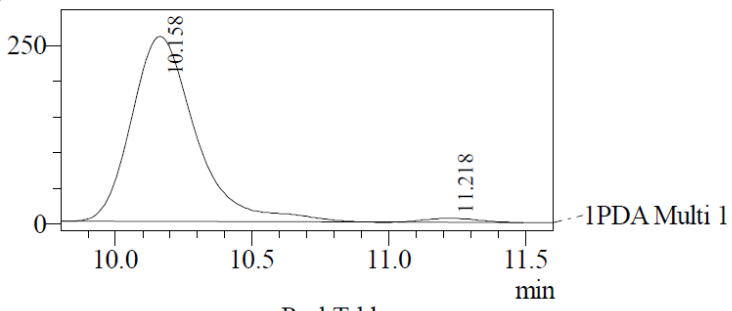

$1 \mathrm{I}$

PDA Ch1 206nm 4nm
\begin{tabular}{|r|r|r|r|}
\hline Peak\# $\#$ & Ret. Time & Area & Area $\%$ \\
\hline 1 & 10.158 & 4254928 & 98.156 \\
\hline 2 & 11.218 & 79930 & 1.844 \\
\hline Total & & 4334858 & 100.000 \\
\hline
\end{tabular}

${ }^{1} \mathrm{H}$ NMR (400 MHz, $\mathrm{CDCl}_{3}: \delta 7.73(\mathrm{~d}, J=7.6 \mathrm{~Hz}, 1 \mathrm{H}), 7.59(\psi \mathrm{t}, J=7.5 \mathrm{~Hz}, 1 \mathrm{H}), 7.42-7.35(\mathrm{~m}, 2 \mathrm{H}), 5.13(\mathrm{~d}, J$ $=9.4 \mathrm{~Hz}, 1 \mathrm{H}), 4.23-4.18(\mathrm{~m}, 1 \mathrm{H}), 2.99(\mathrm{dd}, J=19.0,7.7 \mathrm{~Hz}, 1 \mathrm{H}), 2.39(\mathrm{dd}, J=19.0,4.0 \mathrm{~Hz}, 1 \mathrm{H}), 1.86(\mathrm{~s}, 3 \mathrm{H})$, $1.78(\mathrm{~s}, 3 \mathrm{H})$.

${ }^{13} \mathrm{C} \mathrm{NMR}\left(101 \mathrm{MHz}, \mathrm{CDCl}_{3}\right): \delta 206.5,158.6,136.6,135.0,133.9,127.7,126.7,126.5,123.5,44.6,37.7,25.8$, 18.5.

HRMS (ESI ${ }^{+}$: Calcd for $\mathrm{C}_{13} \mathrm{H}_{15} \mathrm{O}[\mathrm{M}+\mathrm{H}]^{+}:$187.1117. Found: 187.1119 . 
<smiles>CC(C)[C@H]1CC(=O)c2ccccc21</smiles>

\section{(S)-3-i-Propyl-1-indanone (8a)}

The product was isolated by flash chromatography (ethyl acetate/hexanes 1:25) as yellow oil. 24.1 mg, 70\% yield. $93 \%$ ee.

$[\alpha]^{23} \mathrm{D}=-2.8^{\circ}\left(c=0.78, \mathrm{CHCl}_{3}\right)$.

HPLC: Daicel Chiralcel IC-3, $n$-hexane/isopropanol 95/5, flow rate $=0.5 \mathrm{~mL} / \mathrm{min}$.

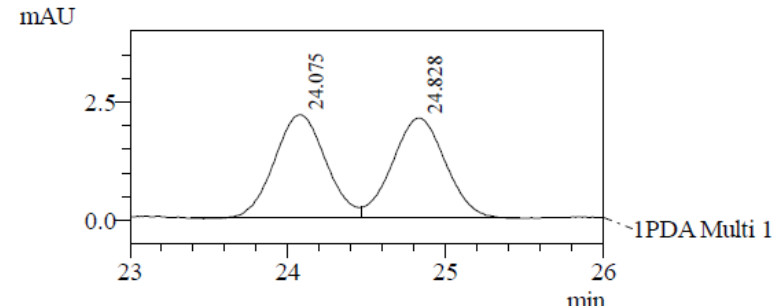

1 PDA Multi $1 / 220 \mathrm{~nm} 4 \mathrm{~nm}$

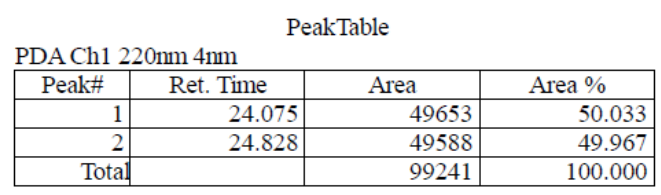

$\mathrm{mAU}$

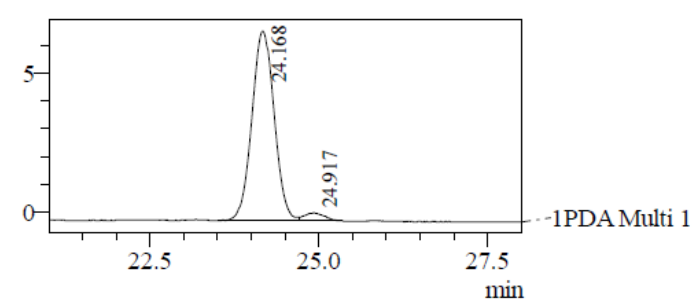

1 PDA Multi $1 / 220 \mathrm{~nm} 4 \mathrm{~nm}$

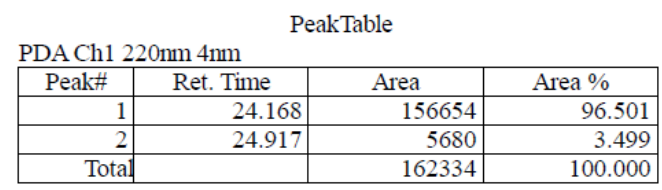

${ }^{1} \mathrm{H}$ NMR (400 MHz, $\left.\mathrm{CDCl}_{3}\right): \delta 7.74(\mathrm{~d}, J=7.8 \mathrm{~Hz}, 1 \mathrm{H}), 7.62-7.58(\mathrm{~m}, 1 \mathrm{H}), 7.49(\mathrm{~d}, J=7.7 \mathrm{~Hz}, 1 \mathrm{H}), 7.38(\psi \mathrm{t}$, $J=7.3 \mathrm{~Hz}, 1 \mathrm{H}), 3.44-3.40(\mathrm{~m}, 1 \mathrm{H}), 2.66(\mathrm{dd}, J=19.2,7.7 \mathrm{~Hz}, 1 \mathrm{H}), 2.46(\mathrm{dd}, J=19.2,3.1 \mathrm{~Hz}, 1 \mathrm{H}), 2.34-2.21$ $(\mathrm{m}, 1 \mathrm{H}), 1.02(\mathrm{~d}, J=6.8 \mathrm{~Hz}, 3 \mathrm{H}), 0.67(\mathrm{~d}, J=6.8 \mathrm{~Hz}, 3 \mathrm{H})$.

${ }^{13} \mathrm{C} \mathrm{NMR}\left(101 \mathrm{MHz}, \mathrm{CDCl}_{3}\right): \delta$ 206.8, 157.9, 137.7, 134.6, 127.6, 126.0, 123.6, 44.3, 38.7, 31.6, 21.2, 16.7. HRMS (ESI ${ }^{+}$: Calcd for $\mathrm{C}_{12} \mathrm{H}_{15} \mathrm{O}[\mathrm{M}+\mathrm{H}]^{+}:$175.1117. Found: 175.1120 .<smiles>O=C1CC(CCc2ccccc2)c2ccccc21</smiles>

\section{(R)-3-Phenethyl -1-indanone (8b)}

The product was isolated by flash chromatography (ethyl acetate/hexanes 1:20) as yellow oil. 28.8 mg, 61\% yield. $81 \%$ ee.

$[\alpha]^{23} \mathrm{D}=+1.2^{\circ}\left(c=0.89, \mathrm{CHCl}_{3}\right)$.

HPLC: Daicel Chiralcel OZ-3, $n$-hexane/isopropanol 95/5, flow rate $0.5 \mathrm{~mL} / \mathrm{min}$. 
mAU

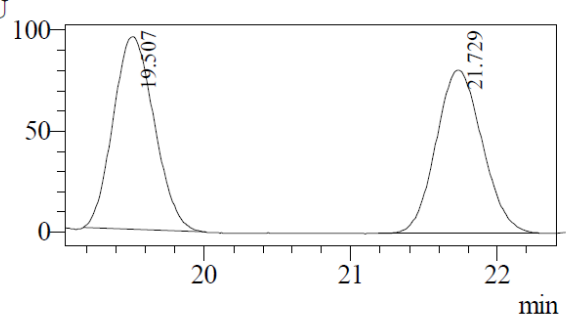

$1 \mathrm{I}$

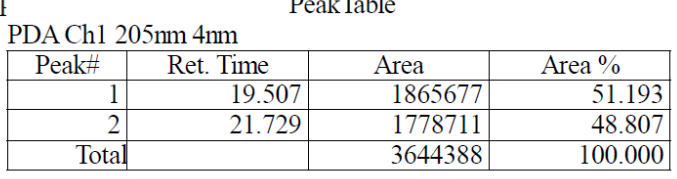

$\mathrm{mAU}$

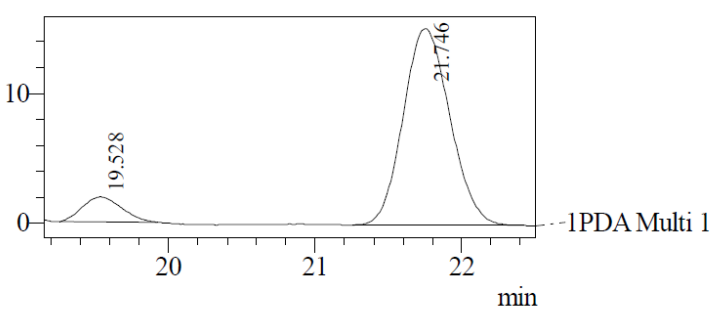

$1 \mathrm{~F}$

PeakTable

PDACh1 $254 \mathrm{~nm} 4 \mathrm{~nm}$
\begin{tabular}{|r|r|r|r|}
\hline Peak\# & Ret. Time & Area & \multicolumn{1}{|c|}{ Area $\%$} \\
\hline 1 & 19.528 & 35141 & 9.467 \\
\hline 2 & 21.746 & 336066 & 90.533 \\
\hline Total & & 371207 & 100.000 \\
\hline
\end{tabular}

${ }^{1} \mathrm{H}$ NMR $\left(400 \mathrm{MHz}, \mathrm{CDCl}_{3}: \delta 7.75(\mathrm{~d}, J=7.7 \mathrm{~Hz}, 1 \mathrm{H}), 7.62-7.58(\psi \mathrm{t}, J=7.2 \mathrm{~Hz}, 1 \mathrm{H}), 7.50(\mathrm{~d}, J=7.7 \mathrm{~Hz}, 1 \mathrm{H})\right.$, 7.38 ( $\psi \mathrm{t}, J=7.4 \mathrm{~Hz}, 1 \mathrm{H}), 7.32-7.23(\mathrm{~m}, 2 \mathrm{H}), 7.22-7.18(\mathrm{~m}, 3 \mathrm{H}), 3.44-3.32(\mathrm{~m}, 1 \mathrm{H}), 2.95(\mathrm{dd}, J=14.1,7.7 \mathrm{~Hz}$

$1 \mathrm{H}), 2.75-2.67(\mathrm{~m}, 2 \mathrm{H}), 2.48-2.40(\mathrm{dd}, J=14.1,7.7 \mathrm{~Hz} 1 \mathrm{H}, 1 \mathrm{H}), 2.32-2.23(\mathrm{~m}, 1 \mathrm{H}), 1.87-1.75(\mathrm{~m}, 1 \mathrm{H})$.

${ }^{13} \mathrm{C} \mathrm{NMR}\left(101 \mathrm{MHz}, \mathrm{CDCl}_{3}\right): \delta 206.3,158.7,141.6,137.0,134.9,128.7,128.5,127.8,126.3,125.7,123.8$, $43.1,38.0,37.9,34.1$.

HRMS (ESI ${ }^{+}$: Calcd for $\mathrm{C}_{17} \mathrm{H}_{17} \mathrm{O}[\mathrm{M}+\mathrm{H}]^{+}: 237.1274$. Found: 237.1285 .<smiles>O=C1CC(C2CCCCC2)c2ccccc21</smiles>

\section{(S)-3-Cyclohexyl-1-indanone (8c)}

The product was isolated by flash chromatography (ethyl acetate/hexanes 1:25) as yellow oil. $27.8 \mathrm{mg}, 65 \%$ yield. $92 \%$ ee.

$[\alpha]^{23}{ }_{\mathrm{D}}=+44.3^{\circ}\left(c=1.22, \mathrm{CHCl}_{3}\right)$.

HPLC: Daicel Chiralcel OZ-3, $n$-hexane/isopropanol 90/10, flow rate $=0.5 \mathrm{~mL} / \mathrm{min}$.

mAU

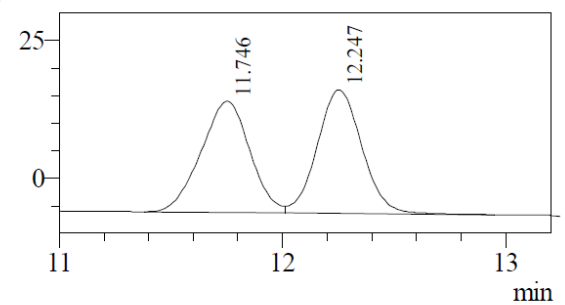

1 PDA Multi 1 / 220nm 4nm

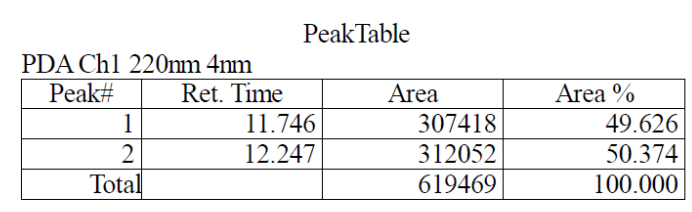

$\mathrm{mAU}$

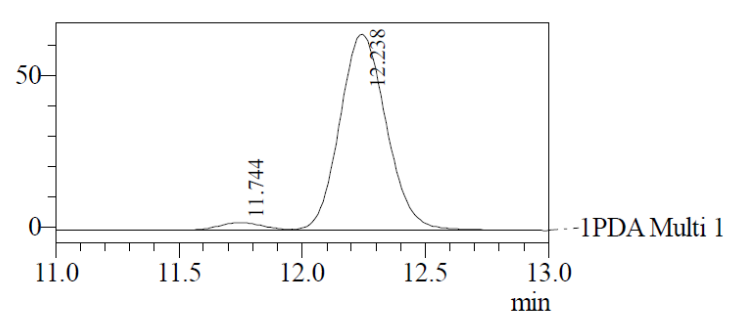

1 PDA Multi 1 / 220nm 4nm

PeakTable

PDACh1 $220 \mathrm{~nm} 4 \mathrm{~nm}$
\begin{tabular}{|r|r|r|r|}
\hline Peak\# & Ret. Time & Area & Area $\%$ \\
\hline 1 & 11.744 & 33080 & 3.735 \\
\hline 2 & 12.238 & 852576 & 96.265 \\
\hline Total & & 885656 & 100.000 \\
\hline
\end{tabular}


${ }^{1} \mathrm{H}$ NMR $\left(400 \mathrm{MHz}, \mathrm{CDCl}_{3}\right): \delta 7.71(\mathrm{~d}, J=7.7 \mathrm{~Hz}, 1 \mathrm{H}), 7.58(\psi \mathrm{td}, J=7.8,1.1 \mathrm{~Hz}, 1 \mathrm{H}), 7.48(\mathrm{dd}, J=7.7,0.6$ $\mathrm{Hz}, 1 \mathrm{H}), 7.35$ ( $\psi \mathrm{t}, J=7.4 \mathrm{~Hz}, 1 \mathrm{H}), 3.40-3.31$ (m, 1H), $2.66(\mathrm{dd}, J=19.1,7.8 \mathrm{~Hz}, 1 \mathrm{H}), 2.50$ (dd, $J=19.1,3.0$ $\mathrm{Hz}, 1 \mathrm{H}), 1.90-1.72(\mathrm{~m}, 3 \mathrm{H}), 1.67-1.65$ (m, 2H), 1.35-0.85 (m, 6H).

${ }^{13} \mathrm{C} \mathrm{NMR}\left(101 \mathrm{MHz}, \mathrm{CDCl}_{3}\right): \delta 206.9,157.7,137.5,134.5,127.5,126.2,123.5,43.9,42.1,39.8,31.8,27.4$, $26.7,26.5,26.4$.

HRMS (ESI ${ }^{+}$): Calcd for $\mathrm{C}_{15} \mathrm{H}_{18} \mathrm{NaO}[\mathrm{M}+\mathrm{Na}]^{+}: 237.1250$. Found: 237.1257 .

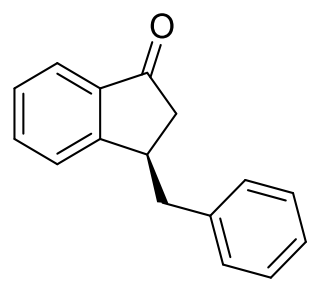

\section{(R)-3-Benzyl-1-indanone (8d)}

The product was isolated by flash chromatography (ethyl acetate/hexanes 1:25) as yellow oil. $24.4 \mathrm{mg}, 55 \%$ yield. $81 \%$ ee.

$[\alpha]^{23} \mathrm{D}=+8.8^{\circ}\left(c=0.62, \mathrm{CHCl}_{3}\right)$.

HPLC: Daicel Chiralcel OJ-H, $n$-hexane/isopropanol 95/5, flow rate $=1.0 \mathrm{~mL} / \mathrm{min}$.

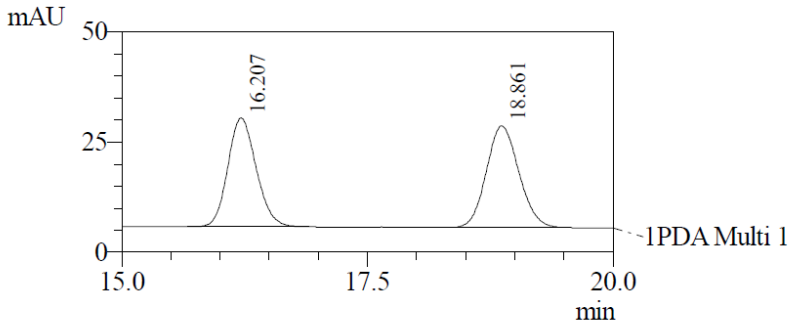

1 PDA Multi $1 / 220 \mathrm{~nm} 4 \mathrm{~nm}$

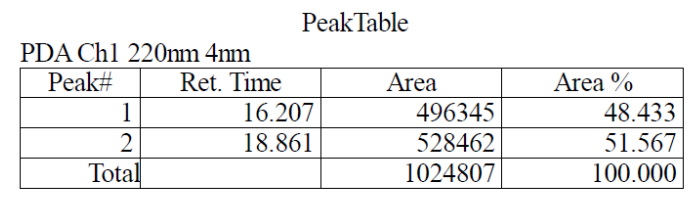

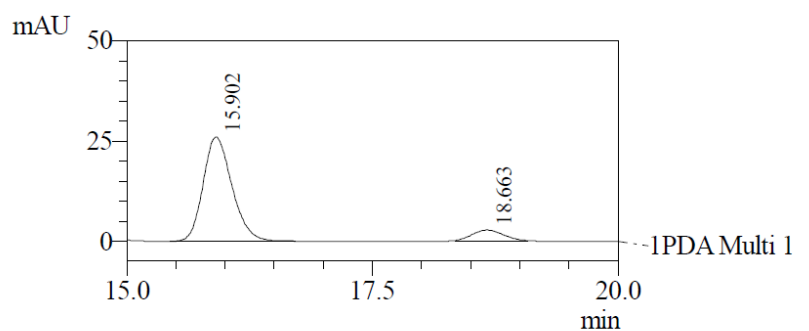

1 PDA Multi $1 / 220 \mathrm{~nm} 4 \mathrm{~nm}$

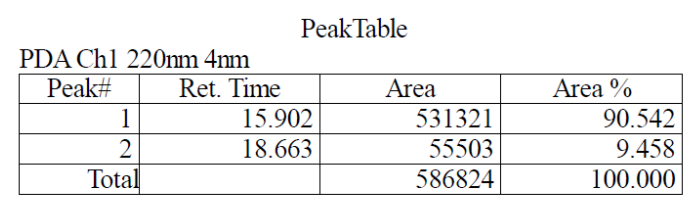

${ }^{1} \mathrm{H}$ NMR $\left(500 \mathrm{MHz}, \mathrm{CDCl}_{3}\right): \delta 7.75(\mathrm{~d}, J=7.6 \mathrm{~Hz}, 1 \mathrm{H}), 7.58(\psi \mathrm{t}, J=7.8 \mathrm{~Hz}, 1 \mathrm{H}), 7.41-7.36(\mathrm{~m}, 2 \mathrm{H}), 7.33-$ $7.29(\mathrm{~m}, 2 \mathrm{H}), 7.24-7.23(\mathrm{~m}, 1 \mathrm{H}), 7.19-7.17(\mathrm{~m}, 2 \mathrm{H}), 3.75-3.70(\mathrm{~m}, 1 \mathrm{H}), 3.18(\mathrm{dd}, J=13.8,6.0 \mathrm{~Hz}, 1 \mathrm{H}), 2.85-$ $2.76(\mathrm{~m}, 2 \mathrm{H}), 2.45(\mathrm{dd}, J=19.1,3.1 \mathrm{~Hz}, 1 \mathrm{H})$.

${ }^{13} \mathrm{C} \mathrm{NMR}\left(101 \mathrm{MHz}, \mathrm{CDCl}_{3}\right): \delta 206.0,158.1,139.5,137.1,134.7,129.2,128.7,127.9,126.7,126.0,123.8$, $43.0,42.5,39.8$.

HRMS (ESI ${ }^{+}$: Calcd for $\mathrm{C}_{16} \mathrm{H}_{15} \mathrm{O}[\mathrm{M}+\mathrm{H}]^{+}: 223.1117$. Found: 223.1113 . 
<smiles>O=C1CC(C2CC2)c2ccccc21</smiles>

\section{(S)-3-Cyclopropyl-1-indanone (8e)}

The product was isolated by flash chromatography (ethyl acetate/hexanes 1:25) as yellow oil. $20.7 \mathrm{mg}, 61 \%$ yield. $84 \%$ ee.

$[\alpha]^{23} \mathrm{D}=-18.3^{\circ}\left(c=0.45, \mathrm{CHCl}_{3}\right)$. Literature value: $[\alpha]^{23} \mathrm{D}=+24^{\circ}\left(c=0.7, \mathrm{CHCl}_{3}\right)$ for a sample of its enantiomer in $54 \%$ ee $(R) .{ }^{4}$

HPLC: Daicel Chiralcel OJ-H, $n$-hexane/isopropanol 99.8/0.2, flow rate $0.5 \mathrm{~mL} / \mathrm{min}$.

maU

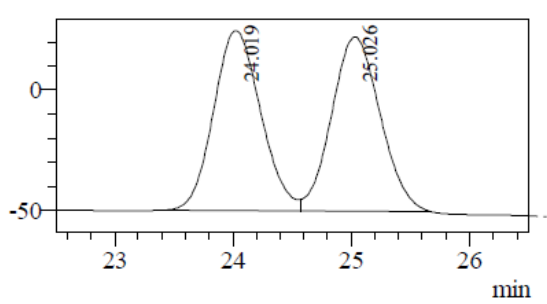

1 PDA Multi 1/207nm 4nm

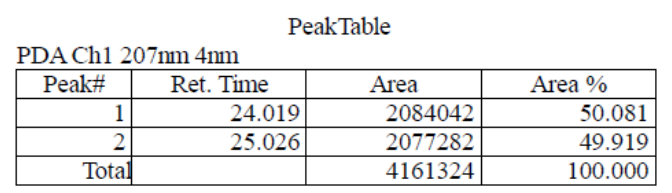

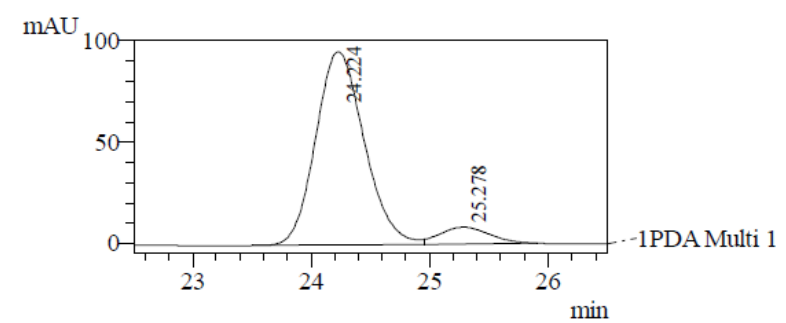

1 PDA Multi $1 / 207 \mathrm{~nm} 4 \mathrm{~nm}$

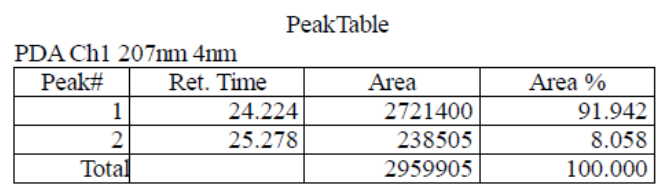

${ }^{1} \mathrm{H}$ NMR $\left(400 \mathrm{MHz}, \mathrm{CDCl}_{3}\right): \delta 7.74(\mathrm{~d}, J=7.7 \mathrm{~Hz}, 1 \mathrm{H}), 7.70(\mathrm{~d}, J=7.7 \mathrm{~Hz}, 1 \mathrm{H}), 7.61(\psi \mathrm{td}, J=7.5,1.1 \mathrm{~Hz}$, $1 \mathrm{H}), 7.40(\psi \mathrm{t}, J=7.4 \mathrm{~Hz}, 1 \mathrm{H}), 2.90(\mathrm{dd}, J=19.0,7.6 \mathrm{~Hz}, 1 \mathrm{H}), 2.77-2.69(\mathrm{~m}, 1 \mathrm{H}), 2.52(\mathrm{dd}, J=19.0,3.4 \mathrm{~Hz}$, $1 \mathrm{H}), 0.98-0.85(\mathrm{~m}, 1 \mathrm{H}), 0.76-0.65(\mathrm{~m}, 1 \mathrm{H}), 0.61-0.47(\mathrm{~m}, 2 \mathrm{H}), 0.39-0.24(\mathrm{~m}, 1 \mathrm{H})$.

${ }^{13} \mathrm{C}$ NMR (101 MHz, $\left.\mathrm{CDCl}_{3}\right): \delta$ 206.4, 158.5, 137.0, 134.8, 127.9, 126.1, 123.6, 43.6, 43.2, 17.1, 4.7, 2.9. HRMS (ESI ${ }^{+}$): Calcd for $\mathrm{C}_{12} \mathrm{H}_{13} \mathrm{O}[\mathrm{M}+\mathrm{H}]^{+}$: 173.0963. Found: 173.0975 .

\section{One-pot reductive cyclization, aldol condensation and dimerization}

A typical procedure: In an argon-filled glove box, $\mathrm{NiBr}_{2}(\mathrm{dme})(3.1 \mathrm{mg}, 0.01 \mathrm{mmol}, 5 \mathrm{~mol} \%)$, ligand $\mathbf{L 8}(0.012$ mmol, $6 \mathrm{~mol} \%)$ and anhydrous DMF $(1.0 \mathrm{~mL})$ were charged into a dry 10-mL Schlenk tube. After stirring for about $10 \mathrm{~min}$ at room temperature, $\mathrm{Li}_{2} \mathrm{CO}_{3}(0.6 \mathrm{mmol}, 44.5 \mathrm{mg}, 3.0$ equiv), Mn powder ( $0.6 \mathrm{mmol}, 33.0 \mathrm{mg}, 3.0$ equiv), $\mathrm{H}_{2} \mathrm{O}(0.2 \mathrm{mmol}, 3.6 \mu \mathrm{L}, 1.0$ equiv), GC standard $n$-dodecane $(20 \mu \mathrm{L})$, dry THF $(1.0 \mathrm{~mL})$ and $(E)-o$ bromochalcone $(0.2 \mathrm{mmol})$ were added sequentially. The reaction mixture was capped tightly and vigorously stirred in a pre-warmed oil bath maintained at $80^{\circ} \mathrm{C}$ for 24 hours. After the mixture was cooled down to RT, it was treated with aldehyde $\left(0.6 \mathrm{mmol}, 3.0\right.$ equiv) and then was vigorously stirred at $90^{\circ} \mathrm{C}$ for 24 hours. The reaction mixture was subjected to flash chromatography with ethyl acetate/hexanes as the eluent. The 
enantioselectivity of the purified product was determined by chiral HPLC analysis using Daicel Chiralcel columns.<smiles>O=C1c2ccccc2[C@H]([C@@H](c2ccccc2)[C@@H](c2ccccc2)[C@H]2C(=O)c3ccccc3[C@@H]2c2ccccc2)[C@@H]1c1ccccc1</smiles>

\section{(2S,2'S,3R,3'R)- 2,2'-[(1S,2S)-1,2-Diphenylethan-1,2-diyl]bis(3-phenyl-1-indanone) (10a)}

The $d r$ of the crude product was estimated to be $>10: 1$ by HPLC analysis. The major diastereomer was isolated by flash chromatography (ethyl acetate/hexanes 1:20) as yellow solid. $35.8 \mathrm{mg}, 60 \%$ yield over 3 steps. $91 \%$ ee. Crystals were obtained by slow evaporation of a solution in ethyl acetate and hexanes. The absolute configuration was determined by X-ray diffraction.

$[\alpha]^{23} \mathrm{D}=+21.2^{\circ}\left(c=0.60, \mathrm{CHCl}_{3}\right)$.

HPLC: Daicel Chiralcel IC-3, $n$-hexane/isopropanol 95/5, flow rate $=0.5 \mathrm{~mL} / \mathrm{min}$.

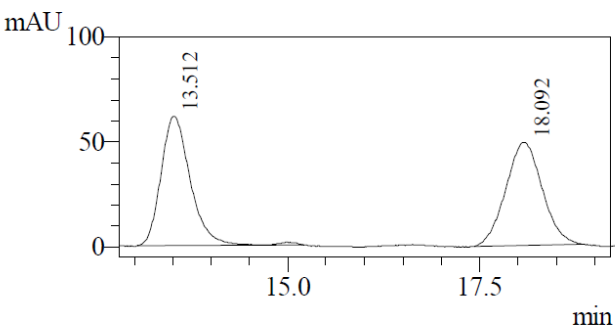

1 PDA Multi $1 / 200 \mathrm{~nm} 4 \mathrm{~nm}$

PDA Ch1 $200 \mathrm{~nm}$ 4nm
\begin{tabular}{|r|c|c|r|}
\hline Peak\# & Ret. Time & Area & Area $\%$ \\
\hline 1 & 13.512 & 1652133 & 50.556 \\
\hline 2 & 18.092 & 1615789 & 49.444 \\
\hline Total & & 3267921 & 100.000 \\
\hline
\end{tabular}

$\mathrm{mAU}$

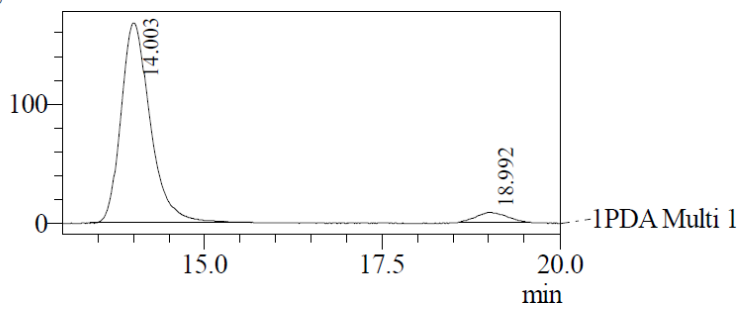

1 PDA Multi $1 / 200 \mathrm{~nm} 4 \mathrm{~nm}$

PDA Chl 200nm 4nm
\begin{tabular}{|r|r|r|r|}
\hline \multicolumn{1}{|c|}{ Peak\# } & Ret. Time & Area & \multicolumn{1}{c|}{ Area $\%$} \\
\hline 1 & 14.003 & 4844363 & 95.324 \\
\hline 2 & 18.992 & 237628 & 4.676 \\
\hline Total & & 5081991 & 100.000 \\
\hline
\end{tabular}

${ }^{1} \mathrm{H}$ NMR (400 MHz, $\left.\mathrm{CDCl}_{3}\right): \delta 7.67$ (d, $\left.J=7.6 \mathrm{~Hz}, 1 \mathrm{H}\right), 7.46-7.34(\mathrm{~m}, 4 \mathrm{H})$, 7.29-7.20 (m, 3H), 6.99-6.85 (m, $6 \mathrm{H}), 4.39-4.35(\mathrm{~m}, 1 \mathrm{H}), 4.07(\mathrm{~d}, J=4.9 \mathrm{~Hz}, 1 \mathrm{H}), 3.87-3.82(\mathrm{~m}, 1 \mathrm{H})$.

${ }^{13} \mathrm{C} \mathrm{NMR}\left(101 \mathrm{MHz}, \mathrm{CDCl}_{3}\right): \delta 207.8,156.9,142.6,141.5,137.5,134.7,129.6,129.0,128.6,128.1,127.5$, $127.3,126.5,126.3,123.2,58.9,50.1,50.0$.

HRMS (ESI ${ }^{+}$): Calcd for $\mathrm{C}_{44} \mathrm{H}_{35} \mathrm{O}_{2}[\mathrm{M}+\mathrm{H}]^{+}:$595.2632. Found: 595.2647.<smiles>O=C1c2ccccc2[C@@H](c2ccccc2)[C@H]1[C@@H](c1cccs1)[C@H]1C(=O)c2ccccc2[C@H]1c1cccs1</smiles> 
The product was isolated by flash chromatography (ethyl acetate/hexanes 1:15) as yellow solid. $33.3 \mathrm{mg}, 55 \%$ yield. $97 \%$ ee.

$[\alpha]^{23} \mathrm{D}=21.2^{\circ}\left(c=0.45, \mathrm{CHCl}_{3}\right)$.

HPLC: Daicel Chiralcel IC-3, $n$-hexane/isopropanol 95/5, flow rate $=0.5 \mathrm{~mL} / \mathrm{min}$.

$$
\mathrm{mAU}
$$

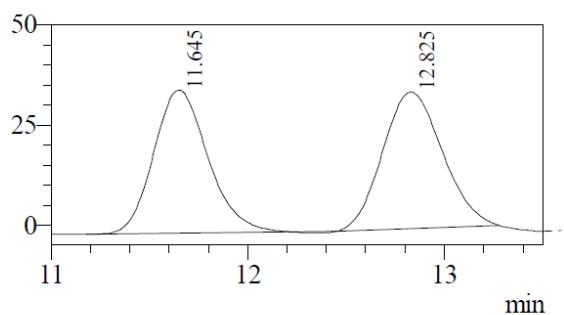

1 PDA Multi 1 / 220nm 4nm

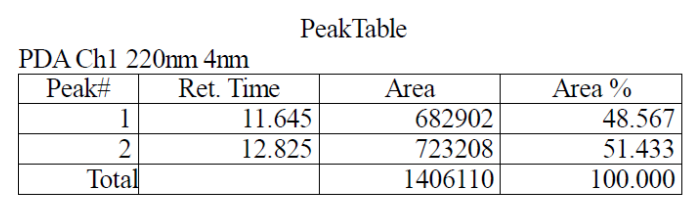

$\mathrm{mAU}$

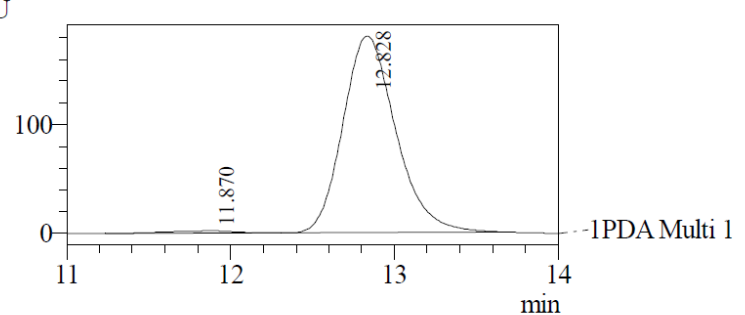

1 PDA Multi $1 / 220 \mathrm{~nm} 4 \mathrm{~nm}$

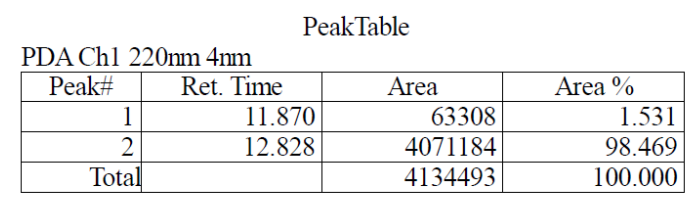

${ }^{1} \mathrm{H} \mathrm{NMR}\left(400 \mathrm{MHz}, \mathrm{CDCl}_{3}\right): \delta 7.68(\mathrm{~d}, J=7.7 \mathrm{~Hz}, 1 \mathrm{H}), 7.45-7.41(\mathrm{~m}, 3 \mathrm{H}), 7.38-7.33(\mathrm{~m}, 1 \mathrm{H}), 7.32-7.27(\mathrm{~m}$, $1 \mathrm{H}), 7.26-7.21(\mathrm{~m}, 2 \mathrm{H}), 7.03(\mathrm{dd}, J=6.4,3.2 \mathrm{~Hz}, 1 \mathrm{H}), 6.88(\mathrm{dd}, J=5.0,1.0 \mathrm{~Hz}, 1 \mathrm{H}), 6.64(\mathrm{dd}, J=5.0,3.5 \mathrm{~Hz}$, $1 \mathrm{H}), 6.60(\mathrm{dd}, J=3.5,1.2 \mathrm{~Hz}, 1 \mathrm{H}), 4.71-4.67(\mathrm{~m}, 1 \mathrm{H}), 4.21(\mathrm{~d}, J=5.1 \mathrm{~Hz}, 1 \mathrm{H}), 3.72-3.66(\mathrm{~m}, 1 \mathrm{H})$.

${ }^{13} \mathrm{C}$ NMR (101 MHz, $\left.\mathrm{CDCl}_{3}\right): \delta 206.9,156.9,144.4,142.3,137.3,134.8,129.0,128.6,127.6,127.4,126.7$, $126.5,126.4,123.9,123.3,59.0,50.1,46.5$.

HRMS (ESI ${ }^{+}$): Calcd for $\mathrm{C}_{40} \mathrm{H}_{31} \mathrm{O}_{2} \mathrm{~S}_{2}[\mathrm{M}+\mathrm{H}]^{+}:$607.1760. Found: 607.1746.<smiles>O=C1c2ccccc2[C@H](c2ccccc2)[C@@H]1[C@@H](c1ccco1)[C@H]1C(=O)c2ccccc2[C@@H]1c1ccco1</smiles>

\section{(2R,2'R,3R,3'R)-2,2'-[(1S,2S)-Di(2-furyl)ethan-1,2-diyl]bis(3-phenyl-1-indanone) (10c)}

The product was isolated by flash chromatography (ethyl acetate/hexanes 1:15) as yellow solid. 29.8 mg, 52\% yield. $90 \%$ ee.

$[\alpha]^{23} \mathrm{D}=-8.7^{\circ}\left(c=0.50, \mathrm{CHCl}_{3}\right)$.

HPLC: Daicel Chiralcel IE-H, $n$-hexane/isopropanol 95/5, flow rate $=0.5 \mathrm{~mL} / \mathrm{min}$. 


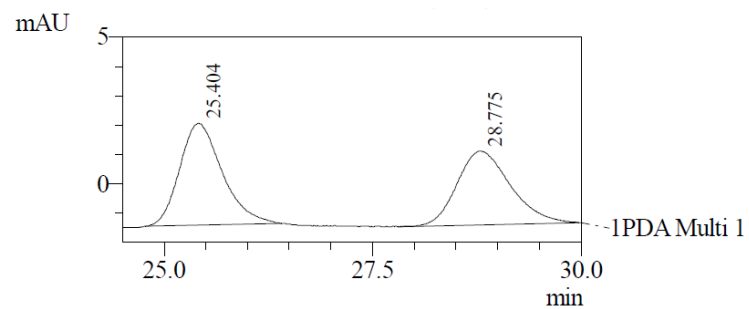

1 PDA Multi $1 / 220 \mathrm{~nm} 4 \mathrm{~nm}$

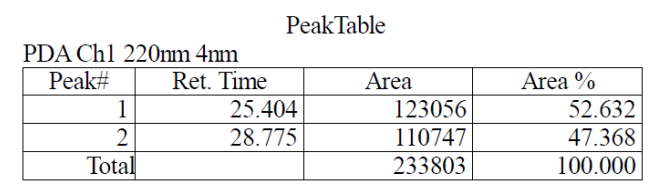

mAU

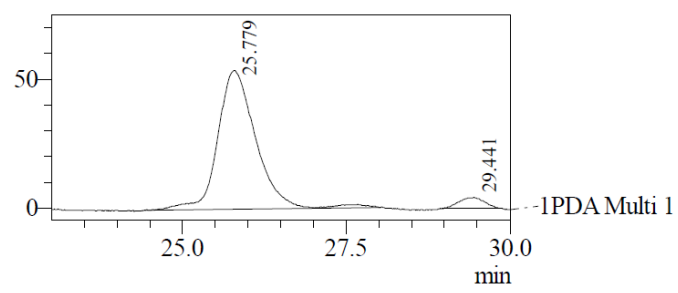

1 PDA Multi $1 / 200 \mathrm{~nm} 4 \mathrm{~nm}$

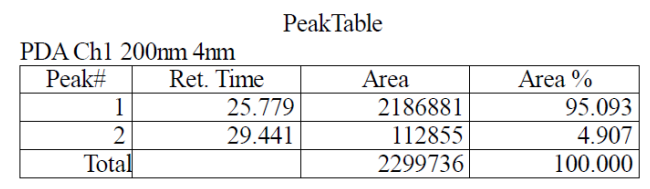

${ }^{1} \mathrm{H}$ NMR (400 MHz, $\left.\mathrm{CDCl}_{3}\right): \delta 7.69$ (d, $\left.J=7.6 \mathrm{~Hz}, 1 \mathrm{H}\right), 7.44(\psi \mathrm{t}, J=7.5 \mathrm{~Hz}, 1 \mathrm{H}), 7.37(\psi \mathrm{t}, J=7.3 \mathrm{~Hz}, 2 \mathrm{H})$, $7.30(\psi \mathrm{t}, J=7.4 \mathrm{~Hz}, 2 \mathrm{H}), 7.19-7.18(\mathrm{~m}, 2 \mathrm{H}), 7.08$ (d, $J=7.7 \mathrm{~Hz}, 1 \mathrm{H}), 6.98-6.95$ (m, 1H), 5.96 (dd, $J=3.1,1.9$ $\mathrm{Hz}, 1 \mathrm{H}), 5.81(\mathrm{~d}, J=3.2 \mathrm{~Hz}, 1 \mathrm{H}), 4.60-4.59(\mathrm{~m}, 1 \mathrm{H}), 4.17$ (d, $J=4.3 \mathrm{~Hz}, 1 \mathrm{H}), 3.55-3.47$ (m, 1H).

${ }^{13} \mathrm{C} \mathrm{NMR}\left(101 \mathrm{MHz}, \mathrm{CDCl}_{3}\right): \delta 206.6,156.2,154.3,142.8,141.4,137.2,134.7,129.0,128.3,127.6,127.2$, $126.6,123.3,109.7,108.1,57.0,50.4,42.3$.

HRMS (ESI ${ }^{+}$): Calcd for $\mathrm{C}_{40} \mathrm{H}_{31} \mathrm{O}_{4}[\mathrm{M}+\mathrm{H}]^{+}:$575.2217. Found: 575.2229.

\section{Asymmetric synthesis toward Resvertrol-based oligomers}<smiles>COc1cc(OC)c(Br)c(C(C)=O)c1</smiles><smiles>COc1ccc(C=O)cc1</smiles>
$\mathrm{NiBr}_{2}(\mathrm{dme}) 5 \mathrm{~mol} \%$ ligand L9 $6 \mathrm{~mol} \%$ $\mathrm{Mn}, \mathrm{Li}_{2} \mathrm{CO}_{3}, \mathrm{H}_{2} \mathrm{O}$ 1:1 DMF/THF $80^{\circ} \mathrm{C}, 24 \mathrm{~h}$

In an argon-filled glove box, 2-bromo-3,5-dimethoxyacetophenone (51.8 mg, $0.2 \mathrm{mmol})$, 4-

methoxybenzaldehyde (27.2 mg, $0.21 \mathrm{mmol}, 1.05$ equiv), $\mathrm{Na}_{2} \mathrm{CO}_{3}$ (63.6 mg, $0.6 \mathrm{mmol}, 3.0$ equiv), GC standard $n$-dodecane $(20 \mu \mathrm{L})$ and anhydrous DMF $(0.5 \mathrm{~mL})$ were charged into a dry $10-\mathrm{mL}$ Schlenk tube. The reaction mixture was capped tightly and vigorously stirred in a pre-warmed oil bath maintained at $140{ }^{\circ} \mathrm{C}$. The reaction was monitored by GC analysis. Upon completion, the mixture was cooled down to rt and passed through a short pug of silica gel to remove the base (washed with a small amount of DMF).

Then $\mathrm{NiBr}_{2}(\mathrm{dme})(3.1 \mathrm{mg}, 0.01 \mathrm{mmol}, 5 \mathrm{~mol} \%)$, L9 (0.012 mmol, $\left.6 \mathrm{~mol} \%\right)$ and anhydrous DMF (0.5 mL) were charged into a new dry 10 -mL Schlenk tube. After stirring for about $10 \mathrm{~min}$ at room temperature, $\mathrm{Li}_{2} \mathrm{CO}_{3}(0.6$ mmol, $44.5 \mathrm{mg}, 3.0$ equiv), Mn powder ( $0.6 \mathrm{mmol}, 33.0 \mathrm{mg}, 3.0$ equiv), $\mathrm{H}_{2} \mathrm{O}$ (0.2 mmol, $3.6 \mu \mathrm{L}, 1.0$ equiv), the 
crude filtrate and dry THF $(1.0 \mathrm{~mL})$ were added sequentially. The reaction mixture was capped tightly and vigorously stirred in a pre-warmed oil bath maintained at $80^{\circ} \mathrm{C}$ for 24 hours. After cooled down to RT, the reaction mixture was subjected to flash chromatography with ethyl acetate/hexanes as the eluent. The product was isolated by flash chromatography (ethyl acetate/hexanes 1:20) as yellow solid. $31.0 \mathrm{mg}, 52 \%$ yield. $96 \%$ ee.

\section{Asymmetric synthesis towards $(R)$-Tolterodine}<smiles>CC(=O)c1ccc(C)cc1Br</smiles>

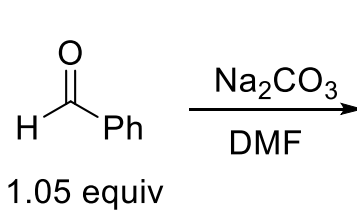

$\mathrm{NiBr}_{2}$ (dme) $5 \mathrm{~mol} \%$ ligand L8 $6 \mathrm{~mol} \%$

$\mathrm{Mn}, \mathrm{Li}_{2} \mathrm{CO}_{3}, \mathrm{H}_{2} \mathrm{O}$

$1: 1 \mathrm{DMF} / \mathrm{THF}$

$80^{\circ} \mathrm{C}, 24 \mathrm{~h}$

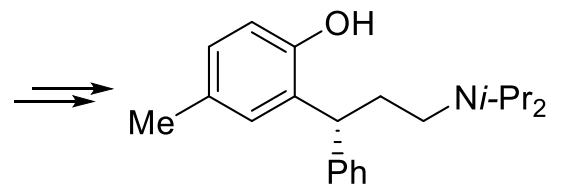

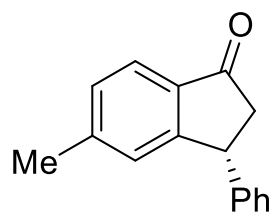

$2 \mathrm{~m} 92 \%$ ee

$64 \%$ yield over 2 steps

$(R)$-Tolterodine

In an argon-filled glove box, 1-(2-bromo-4-methylphenyl)ethan-1-one (42.6 mg, $0.2 \mathrm{mmol})$, benzaldehyde (22.2 mg, $0.21 \mathrm{mmol}, 1.05$ equiv), $\mathrm{Na}_{2} \mathrm{CO}_{3}(63.6 \mathrm{mg}, 0.6 \mathrm{mmol}, 3.0$ equiv), $\mathrm{GC}$ standard $n$-dodecane (20 $\mu \mathrm{L})$, and anhydrous DMF $(0.5 \mathrm{~mL})$ were charged into a dry $10-\mathrm{mL}$ Schlenk tube. The reaction mixture was capped tightly and vigorously stirred in a pre-warmed oil bath maintained at $130^{\circ} \mathrm{C}$. The reaction was monitored by GC analysis. Upon completion, the mixture was cooled down to rt and passed through a short pug of silica gel to remove the base (washed with a small amount of DMF).

Then $\mathrm{NiBr}_{2}(\mathrm{dme})$ (3.1 mg, $\left.0.01 \mathrm{mmol}, 5 \mathrm{~mol} \%\right), \mathbf{L 8}(0.012 \mathrm{mmol}, 6 \mathrm{~mol} \%)$, and anhydrous DMF (0.5 mL) were charged into a new dry $10-\mathrm{mL}$ Schlenk tube. After stirring for about $10 \mathrm{~min}$ at room temperature, $\mathrm{Li}_{2} \mathrm{CO}_{3}$ (0.6 mmol, $44.5 \mathrm{mg}, 3.0$ equiv), Mn powder (0.6 mmol, $33.0 \mathrm{mg}, 3.0$ equiv), $\mathrm{H}_{2} \mathrm{O}(0.2 \mathrm{mmol}, 3.6 \mu \mathrm{L}, 1.0$ equiv), the crude filtrate and THF $(1.0 \mathrm{~mL})$ were added sequentially. The reaction mixture was capped tightly and vigorously stirred in a pre-warmed oil bath maintained at $80^{\circ} \mathrm{C}$ for 24 hours. After cooled down to RT, the reaction mixture was subjected to flash chromatography with ethyl acetate/hexanes as the eluent. The product was isolated by flash chromatography (ethyl acetate/hexanes $1: 20$ ) as yellow solid. $28.5 \mathrm{mg}, 64 \%$ yield. $92 \%$ ee. $[\alpha]^{23} \mathrm{D}=-13.2^{\circ}\left(c=0.5, \mathrm{CHCl}_{3}\right)$.

HPLC: Daicel Chiralcel IC-3, $n$-hexane/isopropanol 95/5, flow rate $=1.0 \mathrm{~mL} / \mathrm{min}$. 
mAU

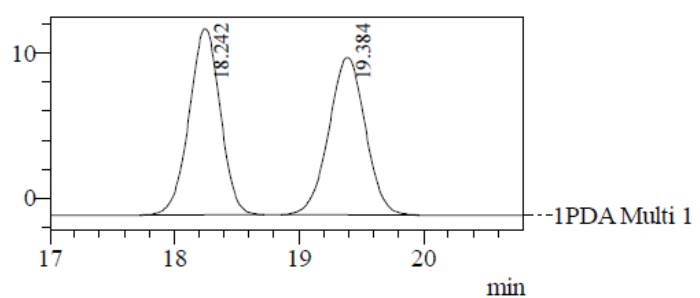

1 PDA Multi $1 / 220 \mathrm{~nm} 4 \mathrm{~nm}$

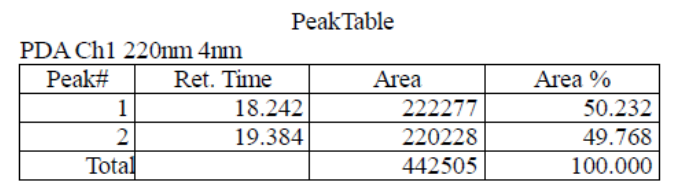

$\mathrm{mAU}$

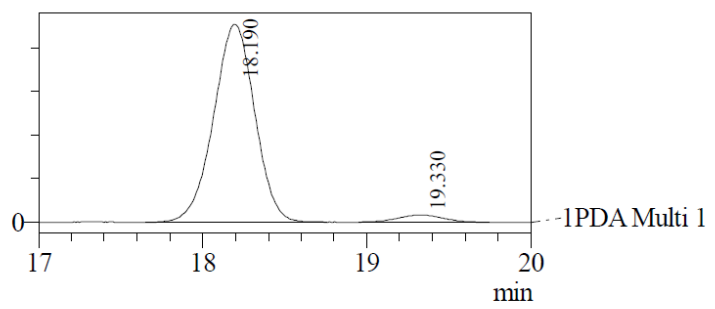

1 PDAMulti $1 / 254 \mathrm{~nm} 4 \mathrm{~nm}$

PDA Ch1 $254 \mathrm{~nm} 4 \mathrm{~nm}$
\begin{tabular}{|r|r|r|r|}
\hline \multicolumn{1}{|c|}{ Peak\# } & Ret. Time & Area & Area $\%$ \\
\hline 1 & 18.190 & 397217 & 96.091 \\
\hline 2 & 19.330 & 16158 & 3.909 \\
\hline Total & & 413375 & 100.000 \\
\hline
\end{tabular}

${ }^{1} \mathrm{H}$ NMR (400 MHz, $\left.\mathrm{CDCl}_{3}\right): \delta 7.70(\mathrm{~d}, J=7.9 \mathrm{~Hz}, 1 \mathrm{H}), 7.32(\psi \mathrm{t}, J=7.3 \mathrm{~Hz}, 2 \mathrm{H}), 7.28-7.20(\mathrm{~m}, 2 \mathrm{H}), 7.15-7.11$ (m, 2H), $7.05(\mathrm{~s}, 1 \mathrm{H}), 4.52(\mathrm{dd}, J=8.0,3.7 \mathrm{~Hz}, 1 \mathrm{H}), 3.21(\mathrm{dd}, J=19.1,8.0 \mathrm{~Hz}, 1 \mathrm{H}), 2.68(\mathrm{dd}, J=19.1,3.8 \mathrm{~Hz}$, $1 \mathrm{H}), 2.37(\mathrm{~s}, 4 \mathrm{H})$.

${ }^{13} \mathrm{C} \mathrm{NMR}\left(101 \mathrm{MHz}, \mathrm{CDCl}_{3}\right): \delta 205.0,158.6,146.5,144.0,134.7,129.3,129.0,127.8,127.2,127.1,123.4$, $47.2,44.5,22.2$.

MS (ESI ${ }^{+}$: Calcd for $\mathrm{C}_{16} \mathrm{H}_{15} \mathrm{O}[\mathrm{M}+\mathrm{H}]^{+}:$223.11. Found: 223.22 .

\section{Asymmetric synthesis of deuteriated indanones}
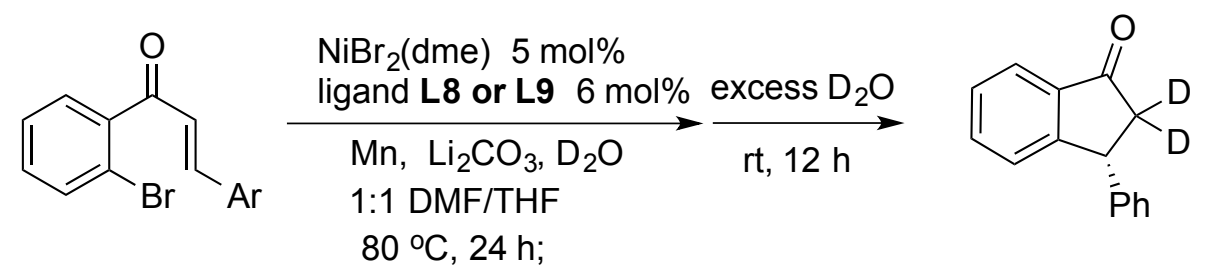

A typical procedure: In an argon-filled glove box, $\mathrm{NiBr}_{2}(\mathrm{dme})(3.1 \mathrm{mg}, 0.01 \mathrm{mmol}, 5 \mathrm{~mol} \%)$, ligand $\mathbf{L 8}$ or $\mathbf{L 9}$ $(0.012 \mathrm{mmol}, 6 \mathrm{~mol} \%)$ and anhydrous DMF $(1.0 \mathrm{~mL})$ were charged into a dry $10-\mathrm{mL}$ Schlenk tube. After stirring for about $10 \mathrm{~min}$ at room temperature, $\mathrm{Li}_{2} \mathrm{CO}_{3}(0.6 \mathrm{mmol}, 44.5 \mathrm{mg}, 3.0$ equiv), Mn powder $(0.6 \mathrm{mmol}$, $33.0 \mathrm{mg}, 3.0$ equiv), $\mathrm{D}_{2} \mathrm{O}(0.2 \mathrm{mmol}, 4.0 \mu \mathrm{L}, 1.0$ equiv), GC standard $n$-dodecane $(20 \mu \mathrm{L})$, THF $(1.0 \mathrm{~mL})$ and (E)-o-bromochalcone $(0.2 \mathrm{mmol})$ were added sequentially. The reaction mixture was capped tightly and vigorously stirred at $80{ }^{\circ} \mathrm{C}$ for 24 hours. After the mixture was cooled down to RT, the product was determined to have $45 \%$ deuterium content at both $\alpha$ positions.

Then $\mathrm{D}_{2} \mathrm{O}(1.0 \mathrm{~mL})$ were added to the crude reaction mixture, which was vigorously stirred at rt overnight. The reaction mixture was subjected to flash chromatography with ethyl acetate/hexanes as the eluent. The enantioselectivity of the purified product was determined by chiral HPLC analysis using Daicel Chiralcel columns. The deuterium content in the purified sample was determined by quantitative NMR spectroscopy using a $90^{\circ}$ pulse sequence with $100 \mathrm{~s}$ acquisition time. The reaction can also be conducted with standard Schlenk technique and a vacuum manifold. 
<smiles>[2H]C1([2H])C(=O)c2ccccc2C1c1ccccc1</smiles>

\section{(R)-3-Phenyl-1-indanone-2,2- $d_{2}\left(2 \mathrm{a}-d_{2}\right)$}

The product was isolated by flash chromatography (ethyl acetate/hexanes 1:20) as yellow solid. $34.8 \mathrm{mg}, 83 \%$ yield. $91 \%$ ee.

$[\alpha]^{23} \mathrm{D}=-45^{\circ}\left(c=0.82, \mathrm{CHCl}_{3}\right)$.

HPLC: Daicel Chiralcel OJ-H, $n$-hexane/isopropanol 95/5, flow rate $=1.0 \mathrm{~mL} / \mathrm{min}$.

$\mathrm{mAU}$

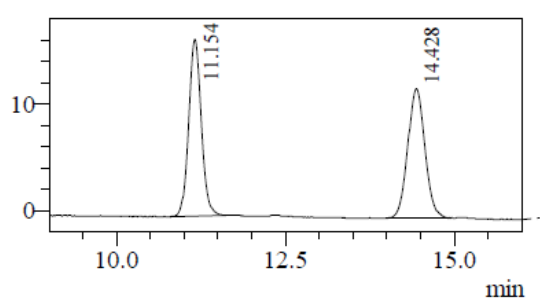

1 PDA Multi $1 / 220 \mathrm{~nm} 4 \mathrm{~nm}$

PeakTable

PDA Ch1 220nm 4nm
\begin{tabular}{|r|c|r|r|}
\hline Peak\# & Ret. Time & Area & Area $\%$ \\
\hline 1 & 11.154 & 219737 & 50.495 \\
\hline 2 & 14.428 & 215425 & 49.505 \\
\hline Total & & 435162 & 100.000 \\
\hline
\end{tabular}

$\mathrm{mAU}$

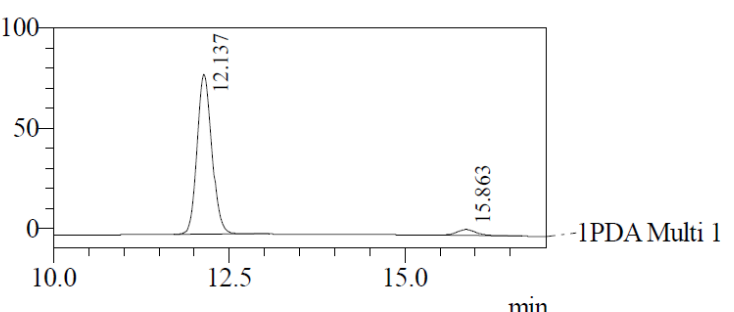

1 PDA Multi $1 / 220 \mathrm{~nm} 4 \mathrm{~nm}$

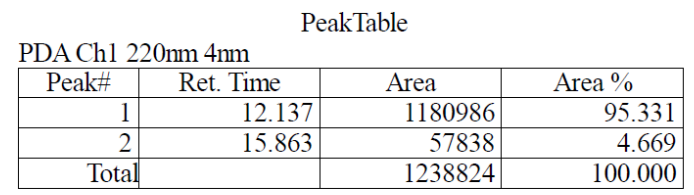

${ }^{1} \mathrm{H}$ NMR $\left(400 \mathrm{MHz}, \mathrm{CDCl}_{3}\right): \delta 7.81(\mathrm{~d}, J=7.7 \mathrm{~Hz}, 1 \mathrm{H}), 7.57(\psi \mathrm{td}, J=7.6,1.1 \mathrm{~Hz}, 1 \mathrm{H}), 7.42(\psi \mathrm{t}, J=7.5 \mathrm{~Hz}$, 1H), 7.35-7.22 (m, 4H), 7.14-7.12 (m, 2H), $4.57(\mathrm{~s}, 1 \mathrm{H}), 3.23-3.20(\mathrm{~m}, 0.04 \mathrm{H}), 2.69-2.67(\mathrm{~m}, 0.04 \mathrm{H})$.

${ }^{13} \mathrm{C} \mathrm{NMR}\left(101 \mathrm{MHz}, \mathrm{CDCl}_{3}\right): \delta 206.2,158.1,143.8,137.0,135.2,129.1,128.0,127.8,127.1,127.0,123.6$, 45.0-46.3 (m), 44.5.

${ }^{2} \mathrm{H}$ NMR (61 MHz, $\left.\mathrm{CDCl}_{3}\right): \delta 3.16,2.69$.

HRMS (ESI ${ }^{+}$): Calcd for $\mathrm{C}_{15} \mathrm{H}_{11} \mathrm{D}_{2} \mathrm{O}[\mathrm{M}+\mathrm{H}]^{+}: 211.1086$. Found: 211.1101.<smiles>[2H]C1([2H])C(=O)c2ccccc2[C@H]1c1ccc(C(C)C)cc1</smiles> 
The product was isolated by flash chromatography (ethyl acetate/hexanes 1:25) as yellow solid. $37.8 \mathrm{mg}, 75 \%$ yield. $94 \%$ ee.

$[\alpha]^{23} \mathrm{D}=-42^{\circ}\left(c=0.65, \mathrm{CHCl}_{3}\right)$.

HPLC: Daicel Chiralcel OJ-H, $n$-hexane/isopropanol 95/5, flow rate $=1.0 \mathrm{~mL} / \mathrm{min}$.

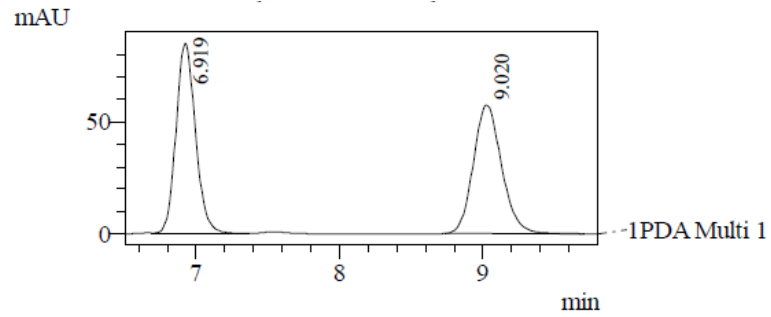

1 PDA Multi $1 / 220 \mathrm{~nm} 4 \mathrm{~nm}$

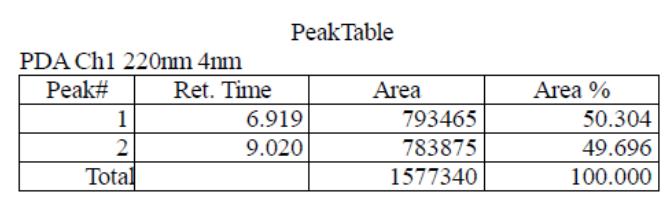

$\mathrm{mAU}$

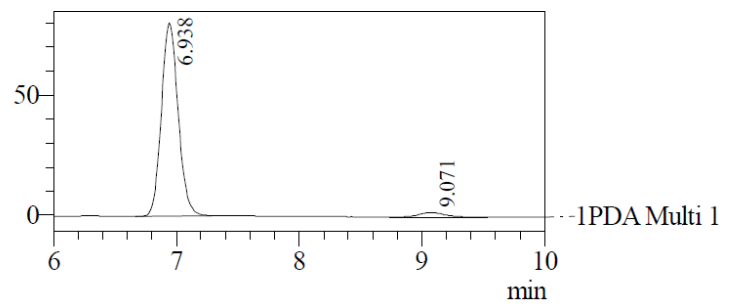

1 PDA Multi $1 / 220 \mathrm{~nm} 4 \mathrm{~nm}$

PDA Ch1 220nm 4nm
\begin{tabular}{|r|r|r|r|}
\hline \multicolumn{1}{|c|}{ Peak\# $\#$ Ret. Time } & Area & Area $\%$ \\
\hline 1 & 6.938 & 769135 & 96.801 \\
\hline 2 & 9.071 & 25419 & 3.199 \\
\hline Total & & 794554 & 100.000 \\
\hline
\end{tabular}

${ }^{1} \mathrm{H}$ NMR $\left(400 \mathrm{MHz}, \mathrm{CDCl}_{3}\right): \delta 7.80(\mathrm{~d}, J=7.7 \mathrm{~Hz}, 1 \mathrm{H}), 7.56(\psi \mathrm{td}, J=7.6,1.2 \mathrm{~Hz}, 31 \mathrm{H}), 7.40(\psi \mathrm{t}, J=7.4 \mathrm{~Hz}$, 1H), 7.29 (d, $J=7.7 \mathrm{~Hz} 1 \mathrm{H}), 7.17$ (d, $J=8.1 \mathrm{~Hz}, 2 \mathrm{H}), 7.04$ (d, $J=8.1 \mathrm{~Hz}, 2 \mathrm{H}), 4.54$ (s, 1H), 3.20-3.18 (m, $0.06 \mathrm{H}), 2.95-2.83(\mathrm{~m}, 1 \mathrm{H}), 2.67-2.66(\mathrm{~m}, 0.06 \mathrm{H}), 1.24(\mathrm{dd}, J=6.9,0.7 \mathrm{~Hz}, 6 \mathrm{H})$.

${ }^{13} \mathrm{C}$ NMR $\left(101 \mathrm{MHz} \mathrm{CDCl}_{3}\right): \delta 206.4,158.2,147.6,140.0,136.8,135.0,127.8,127.5,126.93,126.92,123.4$, 47.1-46.0 (m), 44.0, 33.7, 24.0.

${ }^{2} \mathrm{H}$ NMR (61 MHz, $\left.\mathrm{CDCl}_{3}\right): \delta 3.17,2.67$.

HRMS (ESI ${ }^{+}$): Calcd for $\mathrm{C}_{18} \mathrm{H}_{17} \mathrm{D}_{2} \mathrm{O}[\mathrm{M}+\mathrm{H}]^{+}:$253.1536. Found: 253.1537.<smiles>[2H]C1([2H])C(=O)c2ccccc2C1c1ccc2ccccc2c1</smiles>

\section{(R)-3-(2-Naphthyl)-1-indanone-2,2- $d_{2}\left(2 \mathrm{j}-d_{2}\right)$}

The product was isolated by flash chromatography (ethyl acetate/hexanes 1:15) as yellow solid. $37.2 \mathrm{mg}, 71 \%$ yield. $92 \%$ ee.

$[\alpha]^{23} \mathrm{D}=-134.2^{\circ}\left(c=1.0, \mathrm{CHCl}_{3}\right)$.

HPLC: Daicel Chiralcel IC-3, $n$-hexane/isopropanol 95/5, flow rate $=1.0 \mathrm{~mL} / \mathrm{min}$. 
$\mathrm{mAU}$

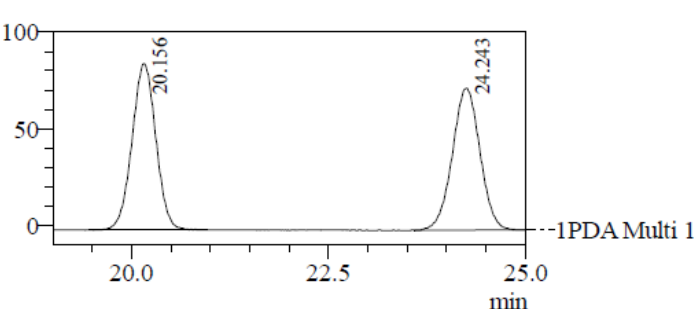

1 PDA Multi $1 / 200 \mathrm{~nm} 4 \mathrm{~nm}$

PDA Ch1 $200 \mathrm{~nm}$ 4nm
\begin{tabular}{|r|c|c|r|}
\hline \multicolumn{1}{|c|}{ Peak\# } & Ret. Time & Area & Area $\%$ \\
\hline 1 & 20.156 & 1807419 & 50.212 \\
\hline 2 & 24.243 & 1792136 & 49.788 \\
\hline Total & & 3599555 & 100.000 \\
\hline
\end{tabular}

$\mathrm{mAU}$

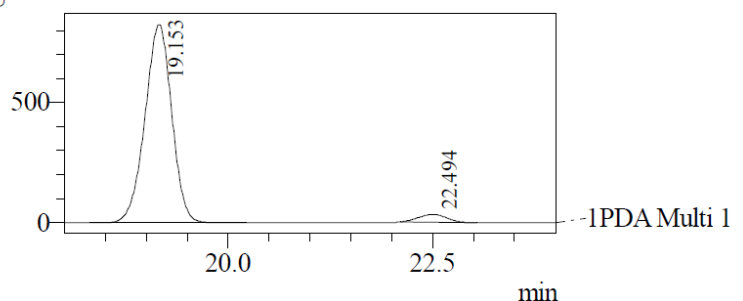

1 PDA Multi $1 / 220 \mathrm{~nm} 4 \mathrm{~nm}$

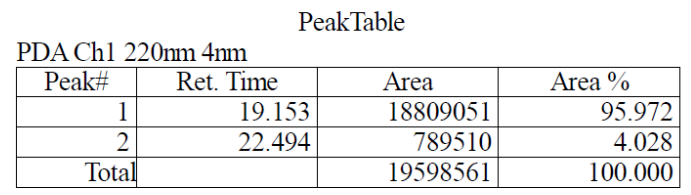

${ }^{1} \mathrm{H}$ NMR (400 MHz, $\left.\mathrm{CDCl}_{3}\right): \delta 7.85(\mathrm{~d}, J=7.7 \mathrm{~Hz}, 1 \mathrm{H}), 7.82-7.77(\mathrm{~m}, 3 \mathrm{H}), 7.66(\mathrm{~s}, 1 \mathrm{H}), 7.57(\mathrm{dd}, J=7.4,1.0$ Hz, 1H), 7.52-7.41 (m, 3H), 7.29 (d, $J=7.7 \mathrm{~Hz}, 1 \mathrm{H}), 7.14$ (dd, $J=8.3,1.7 \mathrm{~Hz}, 1 \mathrm{H}), 4.74$ (s, 1H), 3.29-3.28 (m, $0.03 \mathrm{H}), 2.77-2.75(\mathrm{~m}, 0.03 \mathrm{H})$.

${ }^{13} \mathrm{C}$ NMR (101 MHz, $\left.\mathrm{CDCl}_{3}\right): \delta 206.0,157.9,140.9,136.9,135.2,133.5,132.5,129.0,128.0,127.71,127.63$, 127.0, 126.4, 125.9, 125.5, 123.5, 46.2-45.3 (m), 44.5.

${ }^{2} \mathrm{H} \mathrm{NMR}\left(61 \mathrm{MHz}, \mathrm{CDCl}_{3}\right): \delta 3.30,2.78$.

HRMS (ESI ${ }^{+}$): Calcd for $\mathrm{C}_{19} \mathrm{H}_{13} \mathrm{D}_{2} \mathrm{O}[\mathrm{M}+\mathrm{H}]^{+}: 261.1243$. Found: 261.1259.<smiles>[2H]C1([2H])C(=O)c2ccccc2C1c1ccc(SC)cc1</smiles>

\section{(S)-3-(4-(Methylthio)phenyl)-1-indanone-2,2- $d_{2}\left(4 \mathrm{~d}-d_{2}\right)$}

The product was isolated by flash chromatography (ethyl acetate/hexanes 1:15) as yellow solid. $31.8 \mathrm{mg}, 63 \%$ yield. $97 \%$ ee.

$[\alpha]^{23} \mathrm{D}=-20.1^{\circ}\left(c=1.0, \mathrm{CHCl}_{3}\right)$.

HPLC: Daicel Chiralcel OJ-H, $n$-hexane/isopropanol 90/10, flow rate $=1.0 \mathrm{~mL} / \mathrm{min}$. 


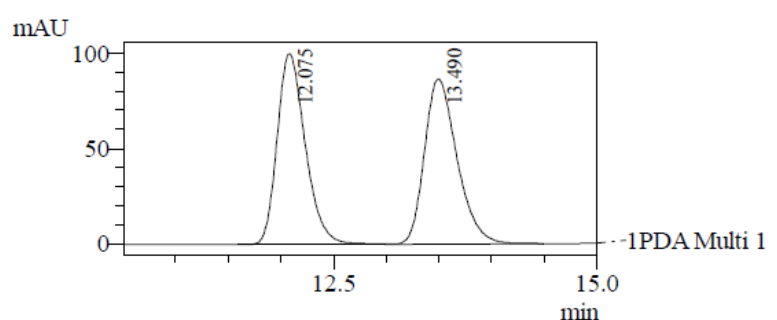

1 PDA Multi $1 / 220 \mathrm{~mm} 4 \mathrm{~nm}$

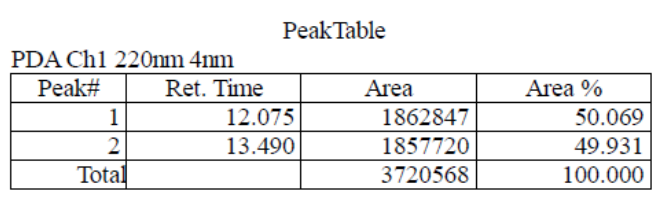

mAU

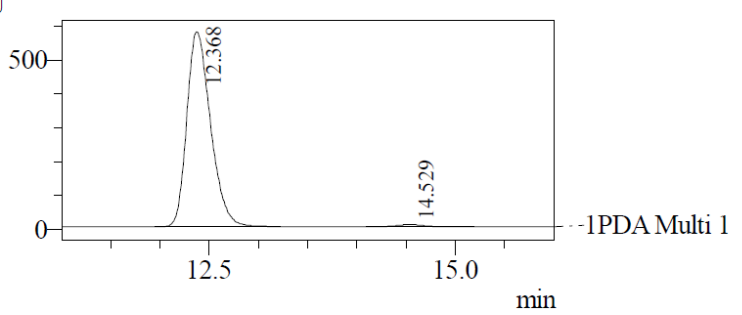

1 PDA Multi $1 / 220 \mathrm{~nm} 4 \mathrm{~nm}$

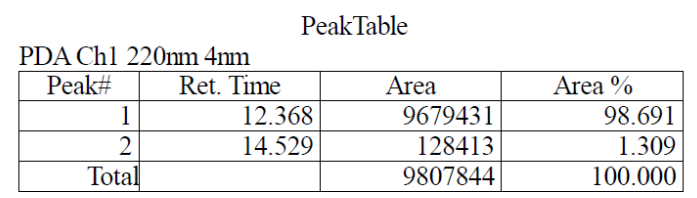

${ }^{1} \mathrm{H}$ NMR $\left(400 \mathrm{MHz}, \mathrm{CDCl}_{3}\right): \delta 7.80(\mathrm{~d}, J=7.4 \mathrm{~Hz}, 1 \mathrm{H}), 7.55(\psi \mathrm{td}, J=7.4,0.9 \mathrm{~Hz}, 1 \mathrm{H}), 7.39(\psi \mathrm{t}, J=7.4 \mathrm{~Hz}$, 1H), $7.30(\mathrm{~d}, J=7.7 \mathrm{~Hz}, 1 \mathrm{H}), 7.26-7.20(\mathrm{~m}, 1 \mathrm{H}), 6.98-6.93(\mathrm{~m}, 1 \mathrm{H}), 6.90-6.85(\mathrm{~m}, 2 \mathrm{H}), 4.88(\mathrm{~s}, 1 \mathrm{H}), 3.75(\mathrm{~s}$, $3 \mathrm{H}), 3.17-3.13(\mathrm{~m}, 0.05 \mathrm{H}), 2.69-2.67(\mathrm{~m}, 0.05 \mathrm{H})$.

${ }^{13} \mathrm{C} \mathrm{NMR}\left(101 \mathrm{MHz}, \mathrm{CDCl}_{3}\right): \delta 206.8,157.9,157.3,137.1,134.7,131.7,128.4,128.1,127.5,126.6,123.3$, 120.7, 110.9, 55.4, 45.1-44.2 (overlapping signals), 39.0.

${ }^{2} \mathrm{H}$ NMR (61 MHz, $\left.\mathrm{CDCl}_{3}\right): \delta 3.24,2.70$.

HRMS (ESI ${ }^{+}$): Calcd for $\mathrm{C}_{16} \mathrm{H}_{13} \mathrm{D}_{2} \mathrm{OS}[\mathrm{M}+\mathrm{H}]^{+}:$257.0964. Found: 257.0958 .<smiles>[2H]C1([2H])C(=O)c2ccccc2C1c1ccc(OC)c(OC)c1</smiles>

\section{(S)-3-(3,4-Dimethoxyphenyl)-1-indanone-2,2- $d_{2}\left(4 f-d_{2}\right)$}

The product was isolated by flash chromatography (ethyl acetate/hexanes 1:10) as yellow solid. $33.5 \mathrm{mg}, 62 \%$ yield. $96 \%$ ee.

$[\alpha]^{23} \mathrm{D}=-33.4^{\circ}\left(c=2.2, \mathrm{CHCl}_{3}\right)$.

HPLC: Daicel Chiralcel AD-H, $n$-hexane/isopropanol 98/2, flow rate $=0.5 \mathrm{~mL} / \mathrm{min}$. 
mAU

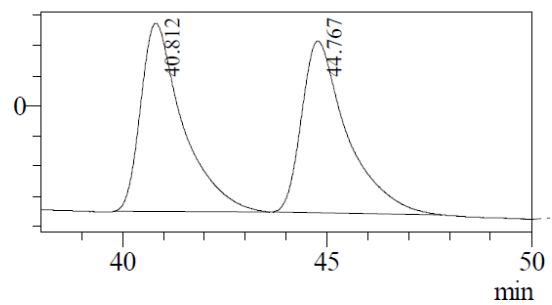

1 PDA Multi $1 / 220 \mathrm{~nm} 4 \mathrm{~nm}$

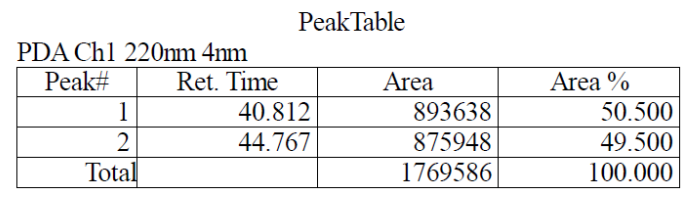

$\mathrm{mAU}$

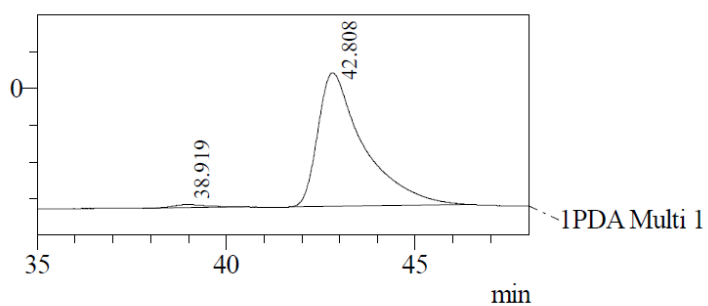

1 PDA Multi $1 / 220 \mathrm{~nm} 4 \mathrm{~nm}$

PeakTable

PDA Ch1 $220 \mathrm{~nm} 4 \mathrm{~nm}$
\begin{tabular}{|r|r|r|r|}
\hline Peak $\#$ & Ret. Time & Area & Area $\%$ \\
\hline 1 & 38.919 & 27214 & 1.754 \\
\hline 2 & 42.808 & 1524361 & 98.246 \\
\hline Total & & 1551575 & 100.000 \\
\hline
\end{tabular}

${ }^{1} \mathrm{H}$ NMR (400 MHz, $\left.\mathrm{CDCl}_{3}\right): \delta 7.82(\mathrm{~d}, J=7.7 \mathrm{~Hz}, 1 \mathrm{H}), 7.61(\psi \mathrm{td}, J=7.6,1.1 \mathrm{~Hz}, 1 \mathrm{H}), 7.44(\psi \mathrm{t}, J=7.5 \mathrm{~Hz}$, 1H), $7.35(\mathrm{~d}, J=8.7 \mathrm{~Hz}, 1 \mathrm{H}), 7.27(\mathrm{~s}, 1 \mathrm{H}), 6.72(\mathrm{~d}, J=8.7 \mathrm{~Hz}, 1 \mathrm{H}), 6.56(\mathrm{~s}, 1 \mathrm{H}), 5.05(\mathrm{~s}, 1 \mathrm{H}), 3.91(\mathrm{~s}, 3 \mathrm{H})$, $3.84(\mathrm{~s}, 3 \mathrm{H}), 3.28-3.25(\mathrm{~m}, 0.06 \mathrm{H}), 2.59-2.53(\mathrm{~m}, 0.06 \mathrm{H})$.

${ }^{13} \mathrm{C}$ NMR (101 MHz, $\left.\mathrm{CDCl}_{3}\right): \delta 205.9,157.1,152.7,145.9,137.4,135.2,134.5,128.8,128.2,127.1,123.7$, $122.8,111.1,60.8,56.2,45.2,41.0-40.8(\mathrm{~m})$.

${ }^{2} \mathrm{H}$ NMR (61 MHz, $\left.\mathrm{CDCl}_{3}\right): \delta 3.27,2.56$.

HRMS (ESI ${ }^{+}$): Calcd for $\mathrm{C}_{17} \mathrm{H}_{15} \mathrm{D}_{2} \mathrm{O}_{3}[\mathrm{M}+\mathrm{H}]^{+}:$271.1298. Found: 271.1308.

VIII. Asymmetric synthesis of (+)-Indatraline, (+)-Indatraline-1- $d_{1}$ and (+)-Indatraline-2,2- $d_{2}$.<smiles>CC(=O)c1ccccc1Br</smiles><smiles>O=Cc1ccc(Cl)c(Cl)c1</smiles><smiles>CCCCC[N+](=O)OCC</smiles><smiles>O=C1C[C@H](c2ccc(Cl)c(Cl)c2)c2ccccc21</smiles>

$92 \%$ ee, $65 \%$ yield<smiles>O=C(/C=C/c1ccc(Cl)c(Cl)c1)c1ccccc1Br</smiles>

$88 \%$ yield

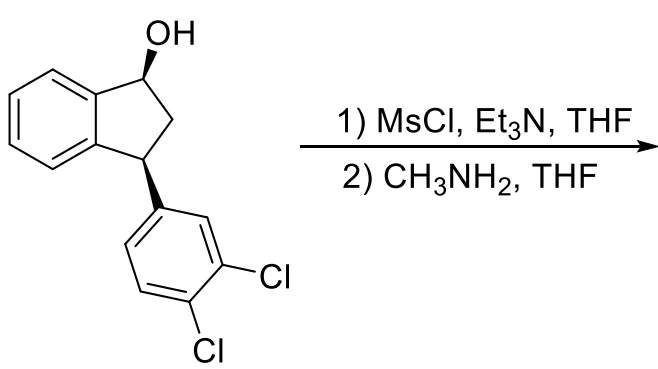

$88 \%$ yield
$\mathrm{NiBr}_{2}$ (dme) $5 \mathrm{~mol} \%$

L9 $6 \mathrm{~mol} \%$ $\mathrm{Mn}$ (3 equiv)

$\mathrm{Li}_{2} \mathrm{CO}_{3}$ (3 equiv)

$\mathrm{H}_{2} \mathrm{O}$ (1.0 equiv)

DMF/THF, $80^{\circ} \mathrm{C}, 24 \mathrm{~h}$<smiles>C[C@H]1C[C@H](c2ccc(Cl)c(Cl)c2)c2ccccc21</smiles>

$71 \%$ yield

(+)-Indatraline

\section{(E)-2-Bromo-3',4'-dichlorochalcone (3h)}

In air, a solution of $\mathrm{NaOH}(1.2 \mathrm{~g}, 30 \mathrm{mmol})$ in water $(5 \mathrm{~mL})$ and ethanol $(30 \mathrm{~mL})$ was cooled to $0{ }^{\circ} \mathrm{C}$ and obromoacetophenone (4.0 g, $20 \mathrm{mmol})$ and 3,4-dichlorobenzaldehyde (3.7g, $21 \mathrm{mmol})$ were slowly added successively. The reaction mixture was allowed to warm to room temperature and kept stirred overnight. After $12 \mathrm{~h}$, the reaction mixture was diluted with diethyl ether $(3 \times 100 \mathrm{~mL})$. The organic phase was separated and 
washed with a saturated aqueous solution of $\mathrm{NH}_{4} \mathrm{Cl}$. The combined organic layers were washed with brine $(2 \times$ $50 \mathrm{~mL}$ ) and concentrated. The solvent was evaporated and the crude material was purified by column chromatography (hexane/EA 15:1) to yield the desired product as a pale yellow solid (88\% yield). We noticed that aldol condensation using $\mathrm{Na}_{2} \mathrm{CO}_{3}$ in DMF resulted in some side products.

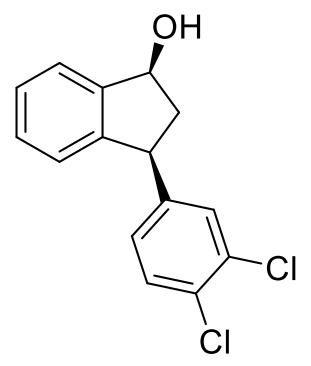

\section{$(1 R, 3 S)-3-(3,4-D i c h l o r o p h e n y l) i n d a n-1-o l ~(4 s)$}

Under nitrogen, substituted indanone ( $135 \mathrm{mg}, 0.5 \mathrm{mmol}, 1.0$ equiv) was dissolved in anhydrous THF (5.0 mL) and cooled to $-10^{\circ} \mathrm{C}$. A $1 \mathrm{M}$ solution of K-Selectride in THF (1.0 mL, $1.0 \mathrm{mmol}, 2.0$ equiv) was added dropwise and the reaction mixture was stirred for $4 \mathrm{~h}$ at $-10{ }^{\circ} \mathrm{C}$. Then, water $(1.0 \mathrm{~mL})$ was added slowly and the solution was stirred for $30 \mathrm{~min}$ at ambient temperature. Later, THF was removed under reduced pressure and the mixture was partitioned between water $(10 \mathrm{~mL})$ and EtOAc $(10 \mathrm{~mL})$. The organic phase was separated and the aqueous layer was re-extracted with EtOAc $(2 \times 10 \mathrm{~mL})$. The organic layers were washed with water $(20$ $\mathrm{mL})$ and then brine $(20 \mathrm{~mL})$, and the solvent was removed under reduced pressure. The residue was subjected to column chromatography $\left(\mathrm{SiO}_{2}\right.$, hexane/EtOAc 4:1) to give cis alcohol (122.9 $\left.\mathrm{mg}, 88 \%\right)$ as a white solid with $\mathrm{dr}>20: 1$.

$[\alpha]^{23} \mathrm{D}=+15.0^{\circ}\left(c=1.2, \mathrm{CHCl}_{3}\right)$.

${ }^{1} \mathrm{H}$ NMR (400 MHz, $\left.\mathrm{CDCl}_{3}\right): \delta 7.45(\mathrm{~d}, J=7.3 \mathrm{~Hz}, 1 \mathrm{H}), 7.36-7.21(\mathrm{~m}, 4 \mathrm{H}), 7.08(\mathrm{dd}, J=8.1,4.3 \mathrm{~Hz}, 1 \mathrm{H}), 6.95$ $(\mathrm{d}, J=8.2 \mathrm{~Hz}, 1 \mathrm{H}), 5.26(\mathrm{t}, J=7.1 \mathrm{~Hz} 1 \mathrm{H}), 4.16-4.10(\mathrm{~m}, 1 \mathrm{H}), 3.01-2.94(\mathrm{~m}, 1 \mathrm{H}), 2.43$ (br s, 1H), 1.91-1.83 $(\mathrm{m}, 1 \mathrm{H})$.

${ }^{13} \mathrm{C} \mathrm{NMR}\left(101 \mathrm{MHz}, \mathrm{CDCl}_{3}\right): \delta 145.2,144.8,144.6,132.6,130.7,130.3,129.7,128.7,127.8,127.7,125.0$, $124.0,74.9,47.8,46.8$.

MS (ESI): Calcd for $\mathrm{C}_{15} \mathrm{H}_{13} \mathrm{Cl}_{2} \mathrm{O}[\mathrm{M}+\mathrm{H}]^{+}:$279.03. Found: 279.12.

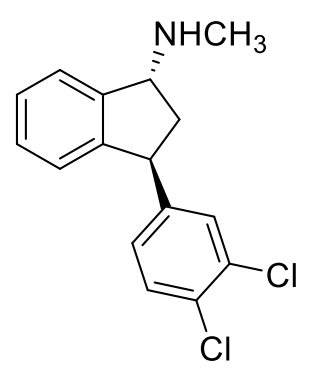

(+)-Indatraline 
Under nitrogen, $\mathrm{Et}_{3} \mathrm{~N}(210 \mu \mathrm{L}, 1.5 \mathrm{mmol}, 5.0$ equiv) was added to a solution of cis alcohol (83.1 $\mathrm{mg}, 0.3 \mathrm{mmol}$, 1.0 equiv) in anhydrous THF $(3.0 \mathrm{~mL})$. The mixture was cooled to $-20{ }^{\circ} \mathrm{C}$, methananesulfonyl chloride $(70.0$ $\mu \mathrm{L}, 0.90 \mathrm{mmol}, 3.0$ equiv) was added dropwise, and the reaction mixture was stirred at $-20{ }^{\circ} \mathrm{C}$ for $1 \mathrm{~h}$. Then, a $1 \mathrm{M}$ solution of methylamine in THF (7.5 mL, $7.5 \mathrm{mmol}, 25.0$ equiv) was added slowly over $30 \mathrm{~min}$. The mixture was then allowed to warm to ambient temperature and stirred overnight. The solvent was removed in vacuo, and water $(10 \mathrm{~mL})$ and EtOAc $(10 \mathrm{~mL})$ were added. The phases were separated and the aqueous layer was re-extracted with EtOAc $(3 \times 50 \mathrm{~mL})$. The combined organic layers were washed with brine $(2 \times 50 \mathrm{~mL})$, dried over anhydrous $\mathrm{Na}_{2} \mathrm{SO}_{4}$, concentrated under reduced pressure, and the residue was subjected to column chromatography $\left(\mathrm{SiO}_{2}\right.$ treated with a drop of $\left.\mathrm{Et}_{3} \mathrm{~N}\right)$ with hexane/ EtOAc 2:3 and then EtOAc to give $(+)-$ Indatraline (67.0 $\mathrm{mg}, 77 \%)$ as colorless oil with $d r>20: 1$.

$[\alpha]^{20}{ }_{\mathrm{D}}=-22.1^{\circ}\left(c=1.1, \mathrm{CHCl}_{3}\right)$. Literature value ${ }^{6}:[\alpha]^{22} \mathrm{D}=-18.9^{\circ}\left(\mathrm{c}=1.1, \mathrm{CHCl}_{3}\right)$.

${ }^{1} \mathrm{H}$ NMR (400 MHz, $\left.\mathrm{CDCl}_{3}\right): \delta 7.45(\mathrm{~d}, J=7.1 \mathrm{~Hz}, 1 \mathrm{H}), 7.36(\mathrm{~d}, J=8.4 \mathrm{~Hz}, 1 \mathrm{H}), 7.30-7.24(\mathrm{~m}, 2 \mathrm{H}), 7.23-7.19$ $(\mathrm{m}, 1 \mathrm{H}), 6.99-6.94(\mathrm{~m}, 2 \mathrm{H}), 4.56(\mathrm{t}, J=7.6 \mathrm{~Hz}, 1 \mathrm{H}), 4.33-4.32(\mathrm{~m}, 1 \mathrm{H}), 3.41(\mathrm{~s}, 1 \mathrm{H}), 2.60-2.47(\mathrm{~m}, 4 \mathrm{H}), 2.27-$ $2.19(\mathrm{~m}, 1 \mathrm{H})$.

${ }^{13} \mathrm{C} \mathrm{NMR}\left(101 \mathrm{MHz}, \mathrm{CDCl}_{3}\right): \delta 145.9,145.4,143.8,132.6,130.6,130.5,130.0,128.8,127.6,127.5,125.5$, 125.1, 63.6, 48.6, 42.8, 34.1.

MS (ESI): Calcd for $\mathrm{C}_{16} \mathrm{H}_{16} \mathrm{Cl}_{2} \mathrm{O}[\mathrm{M}+\mathrm{H}]^{+}:$292.07. Found: 291.98.
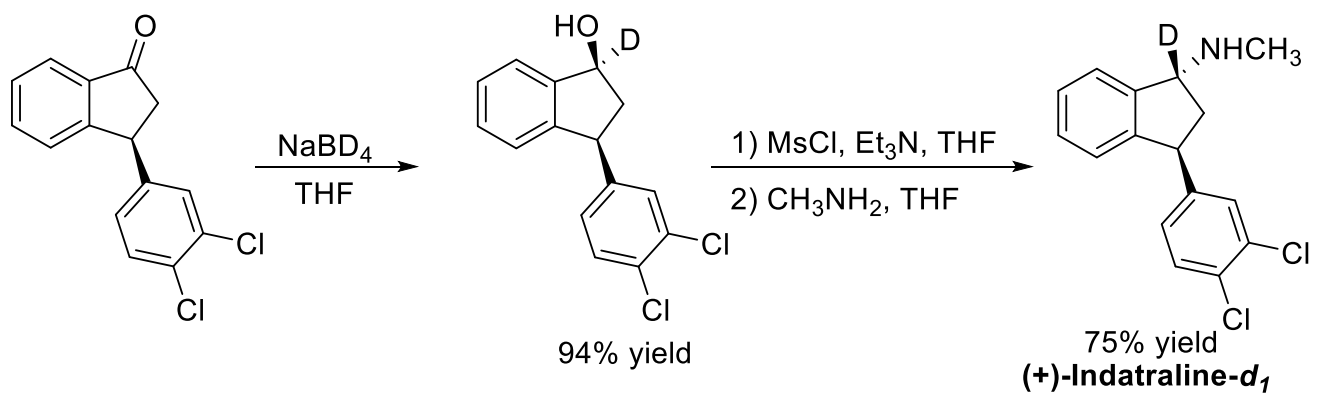

\section{(1R,3S)-3-(3,4-Dichlorophenyl)indan-1-ol-1-d 1}

Under nitrogen, substituted indanone (140 mg, $0.5 \mathrm{mmol}, 1.0$ equiv) was dissolved in THF/ $\mathrm{H}_{2} \mathrm{O}(2.0 \mathrm{~mL} / 0.2$ $\mathrm{mL})$ and cooled to $-5^{\circ} \mathrm{C}$. $\mathrm{NaBD}_{4}(42 \mathrm{mg}, 2.0$ equiv) was added and the reaction mixture was stirred for $4 \mathrm{~h}$ at $0{ }^{\circ} \mathrm{C}$. Then, water $(1.0 \mathrm{~mL})$ was added slowly and the solution was stirred for $30 \mathrm{~min}$ at ambient temperature. Later, THF was removed under reduced pressure and the mixture was partitioned between water $(10 \mathrm{~mL})$ and EtOAc $(30 \mathrm{~mL})$. The organic phase was separated and the aqueous layer was re-extracted with EtOAc $(2 \times 30$ $\mathrm{mL})$. The organic layers were washed with water $(30 \mathrm{~mL})$ and then brine $(30 \mathrm{~mL})$, and the solvent was removed under reduced pressure. The residue was subjected to column chromatography $\left(\mathrm{SiO}_{2}\right.$, hexane/EtOAc $\left.4: 1\right)$ to give cis alcohol (131.5 mg, 94\%) as a white solid with $d r>20: 1$.

$[\alpha]^{23} \mathrm{D}=+11.0^{\circ}\left(c=1.0, \mathrm{CHCl}_{3}\right)$. 
${ }^{1} \mathrm{H}$ NMR (400 MHz, $\left.\mathrm{CDCl}_{3}\right): \delta 7.45(\mathrm{~d}, J=7.3 \mathrm{~Hz}, 1 \mathrm{H}), 7.36-7.21(\mathrm{~m}, 4 \mathrm{H}), 7.08(\mathrm{dd}, J=8.1,4.3 \mathrm{~Hz}, 1 \mathrm{H}), 6.95$ $(\mathrm{d}, J=8.2 \mathrm{~Hz}, 1 \mathrm{H}), 5.26(\mathrm{~s}, 0.02 \mathrm{H}), 4.13(\mathrm{t}, J=8.2 \mathrm{~Hz}, 1 \mathrm{H}), 2.97$ (dd, $J=7.7,5.2 \mathrm{~Hz}, 1 \mathrm{H}), 2.41$ (brs, $1 \mathrm{H}), 1.86$ (dd, $J=9.0,3.5 \mathrm{~Hz}, 1 \mathrm{H})$.

${ }^{13} \mathrm{C} \mathrm{NMR}\left(101 \mathrm{MHz}, \mathrm{CDCl}_{3}\right): \delta 145.2,144.9,144.6,132.6,130.6,130.3,129.9,128.8,127.8,127.7,125.0$, $124.1,74.8-74.5(\mathrm{~m}), 47.7,46.7$.

${ }^{2} \mathrm{H}$ NMR $\left(61 \mathrm{MHz}, \mathrm{CDCl}_{3}\right): \delta 5.29$.

HRMS (ESI ${ }^{+}$): Calcd for $\mathrm{C}_{15} \mathrm{H}_{12} \mathrm{DCl}_{2} \mathrm{O}[\mathrm{M}+\mathrm{Na}]^{+}: 280.0401$. Found: 280.0409 .

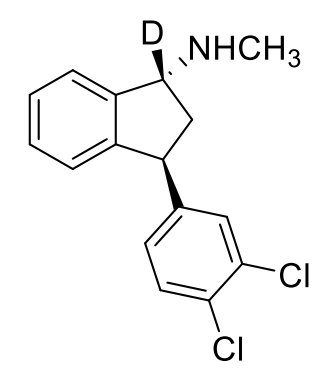

\section{(+)-Indatraline-1- $d_{1}$}

Under nitrogen, $\mathrm{Et}_{3} \mathrm{~N}$ (144 $\mu \mathrm{L}, 1.0 \mathrm{mmol}, 5.0$ equiv) was added to a solution of $c$ is alcohol (56 mg, $0.2 \mathrm{mmol}$, 1.0 equiv) in anhydrous THF $(2.0 \mathrm{~mL})$. The mixture was cooled to $-20^{\circ} \mathrm{C}$, methananesulfonyl chloride (46.0 $\mu \mathrm{L}, 0.60 \mathrm{mmol}, 3.0$ equiv) was added dropwise, and the reaction mixture was stirred at $-20{ }^{\circ} \mathrm{C}$ for $1 \mathrm{~h}$. Then, a $1 \mathrm{M}$ solution of methylamine in THF $(5.0 \mathrm{~mL}, 5.0 \mathrm{mmol}, 25.0$ equiv) was added slowly over $30 \mathrm{~min}$. The mixture was then allowed to warm to ambient temperature and stirred overnight. The solvent was removed in vacuo, and water $(20 \mathrm{~mL})$ and EtOAc $(20 \mathrm{~mL})$ were added. The phases were separated and the aqueous layer was re-extracted with EtOAc $(3 \times 30 \mathrm{~mL})$. The combined organic layers were washed with brine $(2 \times 30 \mathrm{~mL})$, dried over anhydrous $\mathrm{Na}_{2} \mathrm{SO}_{4}$, concentrated under reduced pressure, and the residue was subjected to column chromatography $\left(\mathrm{SiO}_{2}\right.$ treated with a drop of $\left.\mathrm{Et}_{3} \mathrm{~N}\right)$ with hexane/EtOAc 2:3 and then EtOAc to give $(+)$ indatraline- $d_{l}(43.9 \mathrm{mg}, 75 \%)$ as colorless oil with $d r>20: 1$.

$[\alpha]^{23} \mathrm{D}=-8.5^{\circ}\left(c=0.53, \mathrm{CHCl}_{3}\right)$.

${ }^{1} \mathrm{H}$ NMR (400 MHz, $\left.\mathrm{CDCl}_{3}\right): \delta 7.43(\mathrm{~d}, J=8.3 \mathrm{~Hz}, 1 \mathrm{H}), 7.35(\mathrm{~d}, J=8.3 \mathrm{~Hz}, 1 \mathrm{H})$, 7.29-7.22 (m, 3H), 6.98-6.96 $(\mathrm{m}, 2 \mathrm{H}), 4.52(\mathrm{t}, J=7.7 \mathrm{~Hz}, 1 \mathrm{H}), 4.23-4.22(\mathrm{~m}, 0.02 \mathrm{H}), 2.51(\mathrm{~s}, 3 \mathrm{H}), 2.49-2.44(\mathrm{~m}, 1 \mathrm{H}), 2.27-2.22(\mathrm{~m}, 1 \mathrm{H}), 1.91$ $(\mathrm{s}, 1 \mathrm{H})$,

${ }^{13} \mathrm{C}$ NMR (101 MHz, $\left.\mathrm{CDCl}_{3}\right): \delta 145.8,145.7,144.7,132.7,130.6,130.5,130.1,128.6,127.6,127.4,125.4$, 124.9, 63.6-63.2 (m), 48.7, 43.1, 34.1.

${ }^{2} \mathrm{H} \mathrm{NMR}\left(61 \mathrm{MHz}, \mathrm{CDCl}_{3}\right): \delta 4.30$.

HRMS (ESI ${ }^{+}$): Calcd for $\mathrm{C}_{16} \mathrm{H}_{15} \mathrm{DCl}_{2} \mathrm{~N}[\mathrm{M}+\mathrm{H}]^{+}$: 293.0717. Found: 293.0728. 


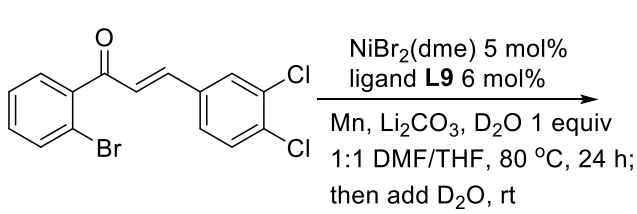

then add $\mathrm{D}_{2} \mathrm{O}$, $\mathrm{rt}$

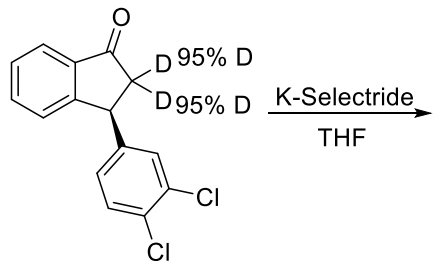

$92 \%$ ee, $60 \%$ yield

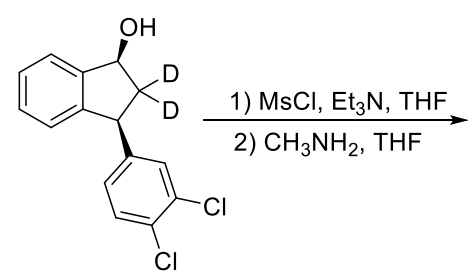

$85 \%$ yield

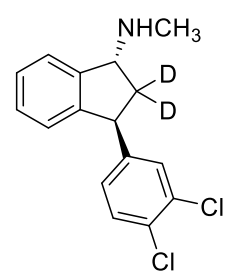

$71 \%$ yield

$(+)$-Indatraline- $d_{2}$

\section{(S)-3-(3,4-Dichlorophenyl)-1-indanone-2,2- $d_{2}\left(4 \mathrm{~h}-d_{2}\right)$}

In an argon-filled glove box, $\mathrm{NiBr}_{2}(\mathrm{dme})(3.1 \mathrm{mg}, 0.01 \mathrm{mmol}, 5 \mathrm{~mol} \%), \mathbf{L 9}(0.012 \mathrm{mmol}, 6 \mathrm{~mol} \%)$ and anhydrous DMF $(1.0 \mathrm{~mL})$ were charged into a dry 10-mL Schlenk tube. After stirring for about 10 min at room temperature, $\mathrm{Li}_{2} \mathrm{CO}_{3}\left(0.6 \mathrm{mmol}, 44.5 \mathrm{mg}, 3.0\right.$ equiv), $\mathrm{Mn}$ powder (0.6 mmol, $33.0 \mathrm{mg}, 3.0$ equiv), $\mathrm{D}_{2} \mathrm{O}(0.2$ mmol, $4.0 \mu \mathrm{L}, 1.0$ equiv), GC standard $n$-dodecane $(20 \mu \mathrm{L})$, THF $(1.0 \mathrm{~mL})$ and $(E)$-2-bromo-3',4'dichlorochalcone $(0.2 \mathrm{mmol})$ were added sequentially. The reaction mixture was capped tightly and vigorously stirred in a pre-warmed oil bath maintained at $80{ }^{\circ} \mathrm{C}$ for 24 hours. After the reaction mixture was cooled down to $\mathrm{RT}, \mathrm{D}_{2} \mathrm{O}(1.0 \mathrm{~mL})$ were added and then the reaction mixture was vigorously stirred at rt overnight. The reaction mixture was subjected to flash chromatography with ethyl acetate/hexanes as the eluent.

The product was isolated by flash chromatography (ethyl acetate/hexanes 1:15) as to give the indannone as yellow solid. $33.5 \mathrm{mg}$, $60 \%$ yield. $92 \%$ ee.

$[\alpha]^{23}{ }_{\mathrm{D}}=+48^{\circ}\left(c=1.0, \mathrm{CHCl}_{3}\right)$. Literature value: $[\alpha]^{24}{ }_{\mathrm{D}}=+49.5^{\circ}\left(c=1.33, \mathrm{CHCl}_{3}\right)$ for a non-deuterated sample of $98 \%$ ee $(S)$.

HPLC: Daicel Chiralcel IC-3, $n$-hexane/isopropanol 95/5, flow rate $=1.0 \mathrm{~mL} / \mathrm{min}$.
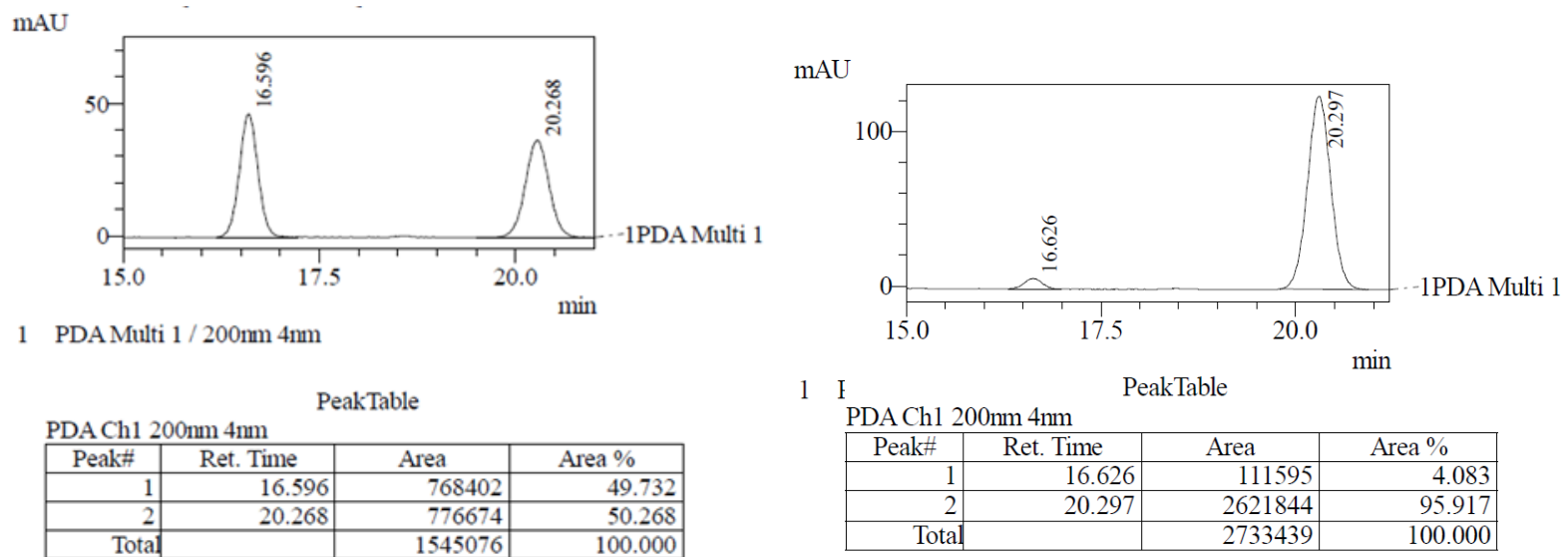
${ }^{1} \mathrm{H}$ NMR $\left(400 \mathrm{MHz}, \mathrm{CDCl}_{3}\right): \delta 7.83(\mathrm{~d}, J=7.7 \mathrm{~Hz}, 1 \mathrm{H}), 7.61(\psi \mathrm{td}, J=7.6,1.2 \mathrm{~Hz}, 1 \mathrm{H}), 7.46(\psi \mathrm{t}, J=7.5 \mathrm{~Hz}$, $1 \mathrm{H}), 7.38(\mathrm{~d}, J=8.3 \mathrm{~Hz}, 1 \mathrm{H}), 7.29-7.25(\mathrm{~m}, 1 \mathrm{H}), 7.23(\mathrm{~d}, J=2.1 \mathrm{~Hz}, 1 \mathrm{H}), 6.95(\mathrm{dd}, J=8.3,2.1 \mathrm{~Hz}, 1 \mathrm{H}), 4.54$ $(\mathrm{s}, 1 \mathrm{H}), 3.22-3.20(\mathrm{~m}, 0.05 \mathrm{H}), 2.61-2.59(\mathrm{~m}, 0.05 \mathrm{H})$.

${ }^{13} \mathrm{C}$ NMR (101 MHz, $\left.\mathrm{CDCl}_{3}\right): \delta 205.0,156.7,144.1,136.9,135.5,133.1,131.3,131.0,129.8,128.5,127.1$, $126.8,123.9,46.6,46.5-45.5(\mathrm{~m}), 43.6$.

${ }^{2} \mathrm{H}$ NMR (61 MHz, $\left.\mathrm{CDCl}_{3}\right): \delta 3.22,2.60$.

HRMS (ESI ${ }^{+}$): Calcd for $\mathrm{C}_{15} \mathrm{H}_{9} \mathrm{Cl}_{2} \mathrm{D}_{2} \mathrm{O}[\mathrm{M}+\mathrm{H}]^{+}:$279.0307. Found: 279.0293 .

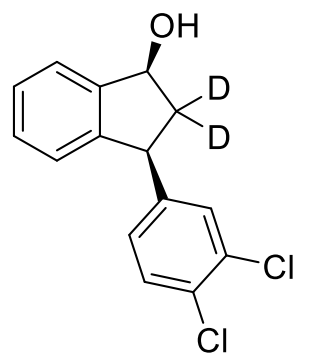

\section{$(1 R, 3 S)-3-(3,4-D i c h l o r o p h e n y l) i n d a n-1-o l-2,2-d_{2}$}

Under nitrogen, substituted indanone $(81.0 \mathrm{mg}, 0.3 \mathrm{mmol}, 1.0$ equiv) was dissolved in anhydrous THF (5.0 mL) and cooled to $-10^{\circ} \mathrm{C}$. A $1 \mathrm{M}$ solution of $\mathrm{K}-$ Selectride in THF $(0.6 \mathrm{~mL}, 0.6 \mathrm{mmol}, 2.0$ equiv) was added dropwise and the reaction mixture was stirred for $4 \mathrm{~h}$ at $-10^{\circ} \mathrm{C}$. Then, water $(1.0 \mathrm{~mL})$ was added slowly and the solution was stirred for $30 \mathrm{~min}$ at ambient temperature. Later, THF was removed under reduced pressure and the mixture was partitioned between water $(10 \mathrm{~mL})$ and EtOAc $(10 \mathrm{~mL})$. The organic phase was separated and the aqueous layer was re-extracted with EtOAc $(2 \times 10 \mathrm{~mL})$. The organic layers were washed with water $(20$ $\mathrm{mL})$ and then brine $(20 \mathrm{~mL})$, and the solvent was removed under reduced pressure. The residue was subjected to column chromatography $\left(\mathrm{SiO}_{2}\right.$, hexane/EtOAc 4:1) to give cis alcohol (71.5 mg, 85\%) as a white solid with $d r>20: 1$.

$[\alpha]^{23} \mathrm{D}=+13.1^{\circ}\left(c=1.1, \mathrm{CHCl}_{3}\right)$.

${ }^{1} \mathrm{H}$ NMR (400 MHz, $\left.\mathrm{CDCl}_{3}\right): \delta 7.49$ (d, $\left.J=7.3 \mathrm{~Hz}, 1 \mathrm{H}\right), 7.39-7.24(\mathrm{~m}, 4 \mathrm{H}), 7.08(\mathrm{dd}, J=8.1,4.3 \mathrm{~Hz}, 1 \mathrm{H}), 6.95$ $(\mathrm{d}, J=8.2 \mathrm{~Hz}, 1 \mathrm{H}), 5.28(\mathrm{~s}, 1 \mathrm{H}), 4.14(\mathrm{~s}, 1 \mathrm{H}), 3.01-2.97(\mathrm{~m}, 0.05 \mathrm{H}), 2.09$ (br s, 1H), 1.90-1.86 (m, 0.05H). ${ }^{13} \mathrm{C} \mathrm{NMR}\left(101 \mathrm{MHz}, \mathrm{CDCl}_{3}\right): \delta 145.3,144.9,144.6,132.7,130.7,130.4,129.8,128.8,127.83,127.80,125.0$, $124.1,74.9,47.6,46.3-45.7(\mathrm{~m})$.

${ }^{2} \mathrm{H}$ NMR (61 MHz, $\left.\mathrm{CDCl}_{3}\right): \delta 3.01,1.89$.

HRMS (ESI ${ }^{+}$): Calcd for $\mathrm{C}_{15} \mathrm{H}_{11} \mathrm{D}_{2} \mathrm{Cl}_{2} \mathrm{O}[\mathrm{M}+\mathrm{H}]^{+}:$281.0464. Found: 281.0482 . 


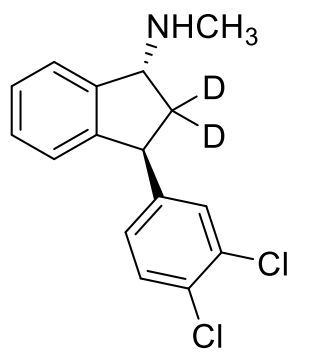

\section{(+)-Indatraline- $d_{2}$}

Under nitrogen, $\mathrm{Et}_{3} \mathrm{~N}(70 \mu \mathrm{L}, 0.5 \mathrm{mmol}, 5.0$ equiv) was added to a solution of cis alcohol (31.0 mg, $0.1 \mathrm{mmol}$, 1.0 equiv) in anhydrous THF $(1.0 \mathrm{~mL})$. The mixture was cooled to $-20^{\circ} \mathrm{C}$, mesyl chloride $(23.0 \mu \mathrm{L}, 0.30 \mathrm{mmol}$, 3.0 equiv) was added dropwise, and the reaction mixture was stirred at $-20{ }^{\circ} \mathrm{C}$ for $1 \mathrm{~h}$. Then, a $1 \mathrm{M}$ solution of methylamine in THF (2.5 mL, $2.5 \mathrm{mmol}, 25.0$ equiv) was added slowly over $30 \mathrm{~min}$. The mixture was then allowed to warm to ambient temperature and stirred overnight. The solvent was removed in vacuo, and water $(10 \mathrm{~mL})$ and EtOAc $(10 \mathrm{~mL})$ were added. The phases were separated and the aqueous layer was re-extracted with EtOAc $(3 \times 10 \mathrm{~mL})$. The combined organic layers were washed with brine $(2 \times 20 \mathrm{~mL})$, dried over anhydrous $\mathrm{Na}_{2} \mathrm{SO}_{4}$, concentrated under reduced pressure, and the residue was subjected to column chromatography $\left(\mathrm{SiO}_{2}\right.$ treated with a drop of $\left.\mathrm{Et}_{3} \mathrm{~N}\right)$ with hexane/ EtOAc 2:3 and then EtOAc to give $(+)-$ indatraline- $d_{2}(21.0 \mathrm{mg}, 71 \%)$ as colorless oil with $d r>20: 1$.

$[\alpha]^{23} \mathrm{D}=-10.3^{\circ}\left(c=0.61, \mathrm{CHCl}_{3}\right)$.

${ }^{1} \mathrm{H}$ NMR (400 MHz, $\left.\mathrm{CDCl}_{3}\right): \delta 7.41(\mathrm{~d}, J=7.1 \mathrm{~Hz}, 1 \mathrm{H}), 7.36(\mathrm{~d}, J=8.4 \mathrm{~Hz}, 1 \mathrm{H}), 7.30-7.24(\mathrm{~m}, 2 \mathrm{H}), 7.23-7.2$ $(\mathrm{m}, 1 \mathrm{H}), 6.98-6.96(\mathrm{~m}, 2 \mathrm{H}), 4.52(\mathrm{~s}, 1 \mathrm{H}), 4.28(\mathrm{~s}, 1 \mathrm{H}), 2.52(\mathrm{~s}, 3 \mathrm{H}), 2.46-2.44(\mathrm{~m}, 0.05 \mathrm{H}), 2.25-2.22(\mathrm{~m}, 0.05 \mathrm{H})$, 2.20 (brs, 1H).

${ }^{13} \mathrm{C}$ NMR $\left(101 \mathrm{MHz}, \mathrm{CDCl}_{3}\right): \delta 145.8,145.6,144.6,132.7,130.6,130.5,130.1,128.6,127.6,127.5,125.5$, 125.0, 63.6, 48.2, 42.8-42.3 (m), 34.1.

${ }^{2} \mathrm{H}$ NMR (61 MHz, $\left.\mathrm{CDCl}_{3}\right): \delta 2.46,2.20$.

HRMS (ESI ${ }^{+}$): Calcd for $\mathrm{C}_{16} \mathrm{H}_{14} \mathrm{D}_{2} \mathrm{Cl}_{2} \mathrm{~N}[\mathrm{M}+\mathrm{H}]^{+}$: 294.0780. Found: 293.0784.

\section{Asymmetric synthesis of (+)-Multisianthol}<smiles>COc1cc(C(C)=O)c(Br)cc1C</smiles><smiles>COc1cc2c(cc1C)C(/C=C(/C)C(=O)O)CC2=O</smiles>

$95 \%$ ee, $75 \%$ yield

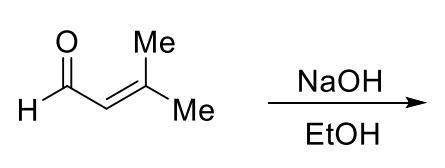<smiles>[X]C([M])=C/C=C/C(=O)c1cc(OC)c(C)cc1Br</smiles>

$5 \mathrm{~h}$ 6i $81 \%$ yield dr 93:7<smiles>C[AsH3]</smiles>

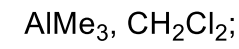

$\mathrm{EtSH}, \mathrm{NaH}, \mathrm{DMF}$<smiles>CC(C)=CC1C[C@@H](C)c2cc(O)c(C)cc21</smiles>

(+)-Multisianthol 


\section{(E)-1-(2-Bromo-5-methoxy-4-methylphenyl)-5-methylhexa-2,4-dien-1-one (5h)}

In air, a solution of $\mathrm{NaOH}(0.3 \mathrm{~g}, 7.5 \mathrm{mmol})$ in water $(1 \mathrm{~mL})$ and ethanol $(20 \mathrm{~mL})$ was cooled to $-10{ }^{\circ} \mathrm{C}$ and 1 (2-bromo-5-methoxy-4-methylphenyl)ethan-1-one (1.21g, $5.0 \mathrm{mmol})$ and 3-methylbut-2-enal (0.63 g, 7.5 mmol) were slowly added successively. The reaction mixture was kept stirred for $3 \mathrm{~h}$. Then, the reaction mixture was diluted with diethyl ether $(3 \times 200 \mathrm{~mL})$, the organic phase was separated and washed with a saturated aqueous solution of $\mathrm{NH}_{4} \mathrm{Cl}$. The combined organic layers were washed with brine $(2 \times 50 \mathrm{~mL})$ and concentrated. The solvent was evaporated and the crude material was purified by column chromatography (hexane/EA 15:1) to yield the desired product as a pale yellow solid. $1.19 \mathrm{~g}, 90 \%$ yield.

${ }^{1} \mathrm{H}$ NMR (400 MHz, $\left.\mathrm{CDCl}_{3}\right): \delta$ 7.48-7.40 (m, 1H), $7.32(\mathrm{~s}, 1 \mathrm{H}), 6.84(\mathrm{~s}, 1 \mathrm{H}), 6.46(\mathrm{~d}, J=15.1 \mathrm{~Hz}, 1 \mathrm{H}), 6.11(\mathrm{~d}$, $J=11.6,1 \mathrm{H}), 3.80(\mathrm{~s}, 3 \mathrm{H}), 2.20(\mathrm{~s}, 3 \mathrm{H}), 1.88(\mathrm{~s}, 3 \mathrm{H}), 1.86(\mathrm{~s}, 3 \mathrm{H})$.

${ }^{13} \mathrm{C} \mathrm{NMR}\left(101 \mathrm{MHz}, \mathrm{CDCl}_{3}\right): \delta 194.5,156.9,149.1,142.2,139.6,134.7,130.8,126.9,124.6,110.6,109.4$, $55.5,26.8,19.2,15.9$.

HRMS (ESI ${ }^{+}$): Calcd for $\mathrm{C}_{15} \mathrm{H}_{18} \mathrm{BrO}_{2}[\mathrm{M}+\mathrm{H}]^{+}: 309.0485$. Found: 309.0492 .

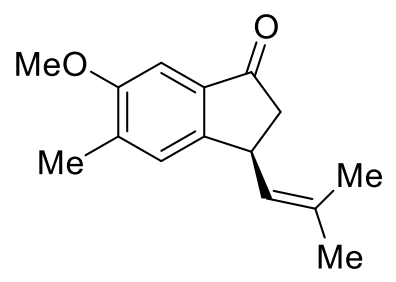

\section{(S)-6-Methoxy-5-methyl-3-isobutenyl-indanone (6h)}

In an argon-filled glove box, $\mathrm{NiBr}_{2}(\mathrm{dme})(124 \mathrm{mg}, 0.40 \mathrm{mmol}, 5 \mathrm{~mol} \%), \mathbf{L 9}(0.48 \mathrm{mmol}, 6 \mathrm{~mol} \%)$ and anhydrous DMF (20.0 mL) were charged into a dry 100-mL Schlenk tube. After stirring for about 10 min at room temperature, $\mathrm{Li}_{2} \mathrm{CO}_{3}$ (1.80 g, $24 \mathrm{mmol}, 3.0$ equiv), Mn powder (1.32 g, 24 mmol, 3.0 equiv), GC standard $n$-dodecane $(800 \mu \mathrm{L})$, THF $(2.0 \mathrm{~mL})$ and $(E)$-1-(2-bromo-5-methoxy-4-methylphenyl)-5-methylhexa-2,4-dien1-one $(8.0 \mathrm{mmol})$ were added sequentially. The reaction mixture was capped tightly and vigorously stirred in a pre-warmed oil bath maintained at $80^{\circ} \mathrm{C}$ for 24 hours. After cooled down to RT, the reaction mixture was subjected to flash chromatography with ethyl acetate/hexanes as the eluent. The product was isolated by flash chromatography (ethyl acetate/hexanes 1:15) to give yellow solid. $1.38 \mathrm{~g}, 75 \%$ yield. 95\% ee. $1 \mathrm{~mol} \%$ nickel procedure at $0.2 \mathrm{mmol} \mathrm{scale:} 100{ }^{\circ} \mathrm{C}$ for 48 hours. $33.0 \mathrm{mg}, 73 \%$ yield. $94 \%$ ee. $[\alpha]^{23} \mathrm{D}=+52.8^{\circ}\left(c=1.27, \mathrm{CHCl}_{3}\right)$.

HPLC: Daicel Chiralcel OD-H, $n$-hexane/isopropanol 98/2, flow rate $=0.3 \mathrm{~mL} / \mathrm{min}$. 
$\mathrm{mAU}$

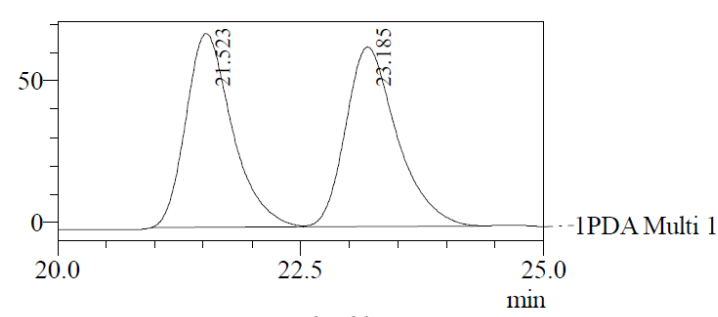

$\mathrm{mAU}$

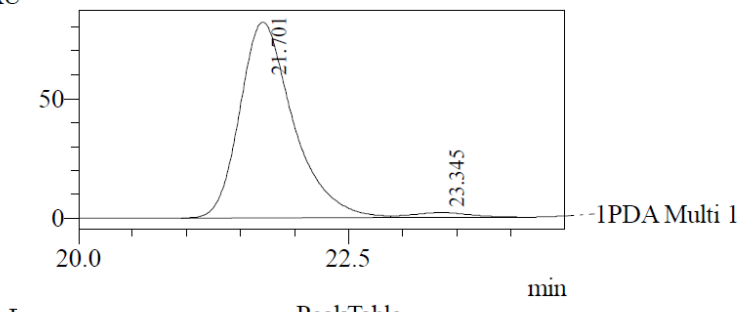

$1 \mathrm{~F}$

${ }^{1} \mathrm{H}$ NMR (400 MHz, $\left.\mathrm{CDCl}_{3}\right): \delta 7.12(\mathrm{~s}, 1 \mathrm{H}), 7.09(\mathrm{~s}, 1 \mathrm{H}), 5.08(\mathrm{~d}, J=9.5 \mathrm{~Hz}, 1 \mathrm{H}), 4.15-4.05(\mathrm{~m}, 1 \mathrm{H}), 3.85(\mathrm{~s}$, 3H), $2.96(\mathrm{dd}, J=19.0,7.4 \mathrm{~Hz}, 1 \mathrm{H}), 2.36(\mathrm{dd}, J=19.0,3.7 \mathrm{~Hz}, 1 \mathrm{H}), 2.29(\mathrm{~s}, 3 \mathrm{H}), 1.84(\mathrm{~s}, 3 \mathrm{H}), 1.77(\mathrm{~s}, 3 \mathrm{H})$.

${ }^{13} \mathrm{C}$ NMR $\left(101 \mathrm{MHz} \mathrm{CDCl}_{3}\right): \delta 206.2,158.1,151.5,136.3,135.6,133.3,127.8,127.1,102.6,55.7,45.1,36.9$, $25.8,22.3,18.4,17.6$.

HRMS (ESI ${ }^{+}$): Calcd for $\mathrm{C}_{15} \mathrm{H}_{18} \mathrm{NaO}_{2}[\mathrm{M}+\mathrm{H}]^{+}: 231.1199$. Found: 231.1201 .<smiles>CCC(=O)OC1CC(C=C(C)C)c2cc(C)c(OC)cc21</smiles>

\section{(1S,3S)-6-Methoxy-5-methyl-1-[2-(methylthio)acetate]-3-isobutenylindane (6i)}

Under nitrogen, to a solution of indanone $(230 \mathrm{mg}, 1.0 \mathrm{mmol}, 1.0$ equiv) in $\mathrm{MeOH}(10 \mathrm{~mL})$ was added solid sodium borohydride $(76 \mathrm{mg}, 2.0 \mathrm{mmol})$ at $-10^{\circ} \mathrm{C}$. The mixture was stirred at the same temperature for $2 \mathrm{~h}$, before it was quenched with $20 \mathrm{~mL}$ of a saturated aqueous solution of $\mathrm{NaHCO}_{3}$. The aqueous layer was extracted with $\mathrm{CH}_{2} \mathrm{Cl}_{2}(50 \mathrm{~mL} \times 3)$. The combined organic layers were dried over $\mathrm{Na}_{2} \mathrm{SO}_{4}$, filtered and concentrated under reduced pressure. The residue was used in the next step without further purification. Under nitrogen, to a stirred solution of 2-(methylthio)acetic acid (224 mg, $4.0 \mathrm{mmol}, 4.0$ equiv) and alcohol (1.0 equiv) in $\mathrm{CH}_{2} \mathrm{Cl}_{2}(5 \mathrm{~mL})$ were added 4-dimethylaminopyridine (122 mg, $1.0 \mathrm{mmol}, 1.0$ equiv) and $N, N^{\prime}-$ dicyclohexylcarbodiimide ( $824 \mathrm{mg}, 4.0 \mathrm{mmol}, 4.0$ equiv). After stirring at ambient temperature for $20 \mathrm{~h}$, the reaction was quenched with a saturated aqueous solution of $\mathrm{NH}_{4} \mathrm{Cl}$. The aqueous layer was extracted with $\mathrm{Et}_{2} \mathrm{O}$ $(50 \mathrm{~mL} \times 3)$. The combined organic layers were dried over $\mathrm{Na}_{2} \mathrm{SO}_{4}$, filtered and concentrated under reduced pressure. Purification by flash chromatography (5\% EtOAc in hexanes) afforded the title compound as colorless oil with $d r=93: 7$ over 2 steps (259 $\mathrm{mg}, 81 \%)$.

$[\alpha]^{23} \mathrm{D}=+37.2^{\circ}\left(c=1.10, \mathrm{CHCl}_{3}\right)$. 
${ }^{1} \mathrm{H}$ NMR (400 MHz, MeOD): $\delta 6.84(\mathrm{~s}, 1 \mathrm{H}), 6.83(\mathrm{~s}, 1 \mathrm{H}), 6.11(\mathrm{t}, J=6.6 \mathrm{~Hz}, 1 \mathrm{H}), 5.15(\mathrm{~d}, J=9.3 \mathrm{~Hz}, 1 \mathrm{H})$, $3.94-3.79(\mathrm{~m}, 1 \mathrm{H}), 3.79(\mathrm{~s}, 3 \mathrm{H}), 3.23(\mathrm{~s}, 2 \mathrm{H}), 2.79-2.72(\mathrm{~m}, 1 \mathrm{H}), 2.19(\mathrm{~s}, 3 \mathrm{H}), 2.15(\mathrm{~s}, 3 \mathrm{H}), 1.80(\mathrm{~s}, 3 \mathrm{H}), 1.76$ (s, 3H), 1.74-1.64 (m, 1H).

${ }^{13} \mathrm{C} \mathrm{NMR}\left(101 \mathrm{MHz}, \mathrm{CDCl}_{3}\right): \delta 170.6,157.5,138.9,138.4,132.2,128.3,128.2,126.4,106.0,78.5,55.6,41.0$, 40.7, 36.1, 25.8, 18.2, 16.7, 16.5 .

HRMS (ESI ${ }^{+}$): Calcd for $\mathrm{C}_{18} \mathrm{H}_{25} \mathrm{O}_{3} \mathrm{~S}[\mathrm{M}+\mathrm{H}]^{+}: 321.1519$. Found: 321.1509 .

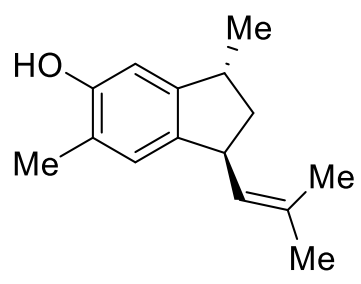

\section{(+)-Multisianthol (6j)}

To a solution of indane $(32.0 \mathrm{mg}, 0.1 \mathrm{mmol})$ in $\mathrm{CH}_{2} \mathrm{Cl}_{2}(2 \mathrm{~mL})$ was added trimethylaluminum $(0.25 \mathrm{~mL}$ of 2.0 $\mathrm{M}$ solution in toluene) at $-78^{\circ} \mathrm{C}$ under argon. The reaction mixture was warmed to $0{ }^{\circ} \mathrm{C}$ over $3 \mathrm{~h}$, quenched with water, and extracted with ether. The organic layer was washed with saturated aq. $\mathrm{NaHCO}_{3}$, and brine, dried over $\mathrm{Na}_{2} \mathrm{SO}_{4}$, and evaporated under reduced pressure. The residue was used in next step without further purification.

The deprotection followed a known procedure. Under nitrogen, $\mathrm{NaH}$ (72 mg, $3.0 \mathrm{mmol}, 30$ equiv, washed with anhydrous hexanes 3 times) and anhydrous DMF $(2.0 \mathrm{~mL})$ were charged into a dry $20-\mathrm{mL}$ Schlenk tube. To this mixture was slowly added a solution of EtSH $(0.23 \mathrm{~mL}, 3.0 \mathrm{mmol}, 30$ equiv) in anhydrous DMF (2.0 mL) at $0{ }^{\circ} \mathrm{C}$ and the resulting yellow solution was stirred for $30 \mathrm{~min}$ at $\mathrm{rt}$. A solution of the crude mixture from the previous step in anhydrous DMF $(2.0 \mathrm{~mL})$ was then added dropwise The reaction mixture turned brownish and was stirred for $8 \mathrm{~h}$ at $120^{\circ} \mathrm{C}$. The mixture was cooled to rt and a saturated solution of $\mathrm{NH}_{4} \mathrm{Cl}$ was added. The mixture was extracted with DCM and the organic phase was washed with $\mathrm{H}_{2} \mathrm{O}$ and brine and dried over anhydrous $\mathrm{Na}_{2} \mathrm{SO}_{4}$. The solvent was removed under reduced pressure. The product was purified by flash chromatography (hexanes/EtOAc 15:1 as eluent) to afford a yellow solid in 80\% yield with $d r=93: 7$ over 2 steps $(17.3 \mathrm{mg})$.

$[\alpha]^{20} \mathrm{D}=+103.0^{\circ}\left(c=1.0, \mathrm{CHCl}_{3}\right)$. Literature value ${ }^{7}:[\alpha]^{23} \mathrm{D}=+95.1^{\circ}\left(c=1.05, \mathrm{CHCl}_{3}\right)$.

${ }^{1} \mathrm{H}$ NMR (400 MHz, $\left.\mathrm{CDCl}_{3}\right): \delta 6.81(\mathrm{~s}, 1 \mathrm{H}), 6.61(\mathrm{~s}, 1 \mathrm{H}), 5.12(\mathrm{~d}, J=9.2 \mathrm{~Hz}, 1 \mathrm{H}), 4.54(\mathrm{br} \mathrm{s}, 1 \mathrm{H}), 4.02-3.92$ (m, 1H), 3.24-3.16 (m, 1H), 2.21 (s, 3H), 1.98-1.89 (m, 2H), 1.78 (br s, 3H), 1.74 (br s, 3H), 1.20 (d, J=7.0 Hz, $3 \mathrm{H})$.

${ }^{13} \mathrm{C} \mathrm{NMR}\left(101 \mathrm{MHz}, \mathrm{CDCl}_{3}\right): 152.9,148.0,138.8,131.3,128.8,126.5,121.8,110.2,42.6,41.7,38.2,26.0$, $21.1,18.3,15.9$.

HRMS (ESI ${ }^{+}$: Calcd for $\mathrm{C}_{15} \mathrm{H}_{22} \mathrm{O}[\mathrm{M}+\mathrm{H}]^{+}: 217.1587$. Found: 217.1593 . 
Another synthetic route using nickel-catalyzed methylation was also attempted, but it led to a mixture of both cis- and trans-isomers. The best result is described below.

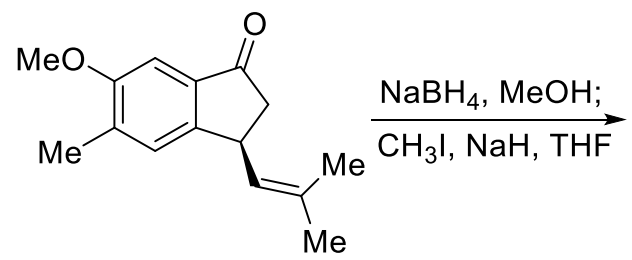

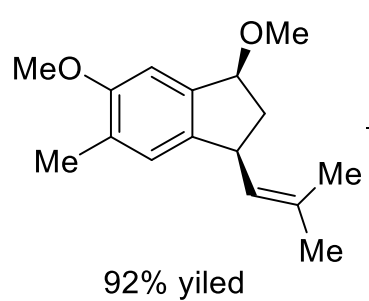

$92 \%$ yiled

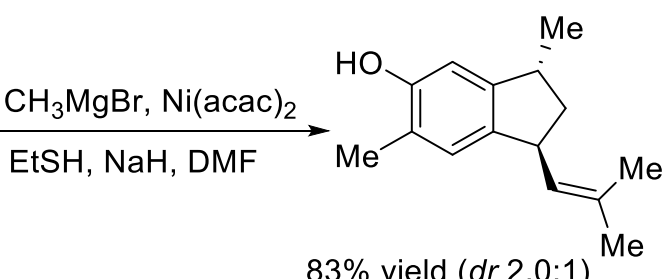

(+)-Multisianthol

\section{(1S,3S)-1,6-Dimethoxy-5-methyl-3-isobutenylindane (6i')}

Under nitrogen, to a solution of indanone $(230 \mathrm{mg}, 1.0 \mathrm{mmol}, 1.0$ equiv) in $\mathrm{MeOH}(10 \mathrm{~mL})$ was added solid sodium borohydride $(76 \mathrm{mg}, 2.0 \mathrm{mmol})$ at $-10{ }^{\circ} \mathrm{C}$. The mixture was stirred at the same temperature for $2 \mathrm{~h}$, before it was quenched with $20 \mathrm{~mL}$ of a saturated aqueous solution of $\mathrm{NaHCO}_{3}$. The aqueous layer was extracted with $\mathrm{CH}_{2} \mathrm{Cl}_{2}(50 \mathrm{~mL} \times 3)$. The combined organic layers were dried over $\mathrm{Na}_{2} \mathrm{SO}_{4}$, filtered and concentrated under reduced pressure. The residue was used in the next without further purification.

Under nitrogen, to a suspension of $\mathrm{NaH}(48.0 \mathrm{mg}, 2.0 \mathrm{mmol}$, washed with hexane in glove box before use) in dry THF $(5.0 \mathrm{~mL})$ was added a solution of the alcohol in dry THF $(5 \mathrm{~mL})$. The mixture was stirred at room temperature for $30 \mathrm{~min}$. MeI $(0.13 \mathrm{~mL}, 2.0 \mathrm{mmol})$ was added and stirring was continued for $12 \mathrm{~h}$ at $\mathrm{rt}$. The reaction was quenched with a saturated aqueous solution of $\mathrm{NH}_{4} \mathrm{Cl}$. The aqueous layer was extracted with $\mathrm{CH}_{2} \mathrm{Cl}_{2}(50 \mathrm{~mL} \times 3)$. The combined organic layers were dried over $\mathrm{Na}_{2} \mathrm{SO}_{4}$, filtered and concentrated under reduced pressure. Purification by flash chromatography (5\% EtOAc in hexanes) afforded the title compound as colorless oil with $\mathrm{dr}>30: 1$ over 2 steps (226 mg, 92\%).

$[\alpha]^{23}{ }_{\mathrm{D}}=+90.3^{\circ}\left(c=1.05, \mathrm{CHCl}_{3}\right)$.

${ }^{1} \mathrm{H}$ NMR (400 MHz, $\left.\mathrm{CDCl}_{3}\right): \delta 6.88(\mathrm{~s}, 2 \mathrm{H}), 5.22-5.18(\mathrm{~m}, 1 \mathrm{H}), 4.83(\mathrm{t}, J=7.0 \mathrm{~Hz}, 1 \mathrm{H}), 4.83(\mathrm{t}, J=7.0 \mathrm{~Hz}$, $1 \mathrm{H}), 3.86(\mathrm{~s}, 3 \mathrm{H}), 3.84-3.77(\mathrm{~m}, 1 \mathrm{H}), 3.49(\mathrm{~s}, 3 \mathrm{H}), 2.75-2.66(\mathrm{~m}, 1 \mathrm{H}), 2.23(\mathrm{~s}, 3 \mathrm{H}), 1.81(\mathrm{~s}, 6 \mathrm{H}), 1.66(\mathrm{ddd}, J=$ $12.5,8.4,7.4 \mathrm{~Hz}, 1 \mathrm{H})$.

${ }^{13} \mathrm{C}$ NMR (101 MHz, $\left.\mathrm{CDCl}_{3}\right): \delta 157.3,141.4,137.4,132.0,128.6,127.0,126.2,105.7,8$ 3.7, 56.3, 55.6, 40.6, $40.5,25.8,18.2,16.6$.

HRMS (ESI ${ }^{+}$: Calcd for $\mathrm{C}_{16} \mathrm{H}_{23} \mathrm{O}_{2}[\mathrm{M}+\mathrm{H}]^{+}:$247.1693. Found: 247.1692.

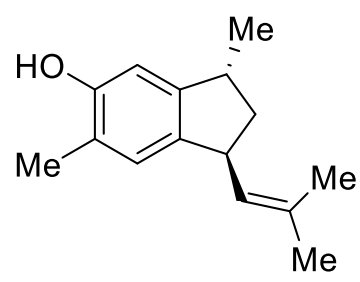




\section{(+)-Multisianthol (6j)}

A typical procedure for methylation: In an argon-filled glove box, Ni(acac)2 $2.6 \mathrm{mg}, 10 \mathrm{~mol} \%)$, dppb (8.5 mg, $20 \mathrm{~mol} \%)$ and dry toluene $(0.5 \mathrm{~mL})$ were charged into a dry $10-\mathrm{mL}$ Schlenk tube. After stirring for about 10 $\mathrm{min}$ at room temperature, $(1 S, 3 S)$-1,6-dimethoxy-5-methyl-3-isobutenylindane (0.1 mmol, $24.6 \mathrm{mg}), 3.0 \mathrm{M}$ solution of $\mathrm{MeMgBr}$ in $\mathrm{Et}_{2} \mathrm{O}(134 \mu \mathrm{L}, 0.4 \mathrm{mmol}$, Aldrich), and GC standard $n$-dodecane $(10 \mu \mathrm{L})$ were added sequentially. The reaction mixture was capped tightly and vigorously stirred in a pre-warmed oil bath maintained at $60{ }^{\circ} \mathrm{C}$ for 36 hours. After the reaction mixture was cooled down to rt, it was quenched with EtOAc and a saturated aqueous solution of $\mathrm{NH}_{4} \mathrm{Cl}$. The aqueous layer was extracted with $\mathrm{CH}_{2} \mathrm{Cl}_{2}(50 \mathrm{~mL} \times 3)$. The combined organic layers were dried over $\mathrm{Na}_{2} \mathrm{SO}_{4}$, filtered and concentrated under reduced pressure. The yield and the dr of the crude product were determined by GC.

The deprotection followed a known procedure. Under nitrogen, $\mathrm{NaH}(0.12 \mathrm{~g}, 5.0 \mathrm{mmol}, 50$ equiv, washed with anhydrous hexanes 3 times) and anhydrous DMF $(2.0 \mathrm{~mL})$ were charged into a dry $20-\mathrm{mL}$ Schlenk tube. To this mixture was slowly added a solution of EtSH $(0.36 \mathrm{~mL}, 5.0 \mathrm{mmol}, 50$ equiv) in anhydrous DMF (1.0 mL) at $0{ }^{\circ} \mathrm{C}$ and the resulting yellow solution was stirred for $30 \mathrm{~min}$ at $\mathrm{rt}$. A crude mixture from the previous step in anhydrous DMF $(2.0 \mathrm{~mL})$ was then added dropwise. The reaction mixture turned brownish and was stirred for 8 $\mathrm{h}$ at $120^{\circ} \mathrm{C}$. The mixture was cooled down to $\mathrm{rt}$ and a saturated solution of $\mathrm{NH}_{4} \mathrm{Cl}$ was added. The mixture was extracted with DCM and the organic phase was washed with $\mathrm{H}_{2} \mathrm{O}$ and brine and dried over anhydrous $\mathrm{Na}_{2} \mathrm{SO}_{4}$. The solvent was removed under reduced pressure. The two isomers were purified by flash chromatography (hexanes/EtOAc 15:1 as eluent) to afford 83\% combined yield (18 mg). The two isomers can be separated by careful chromatography to afford (+)-Multisianthol as a yellow solid.

\section{Table S7: Optimization of nickel-catalyzed methylation with $\mathrm{MeMgBr}$}
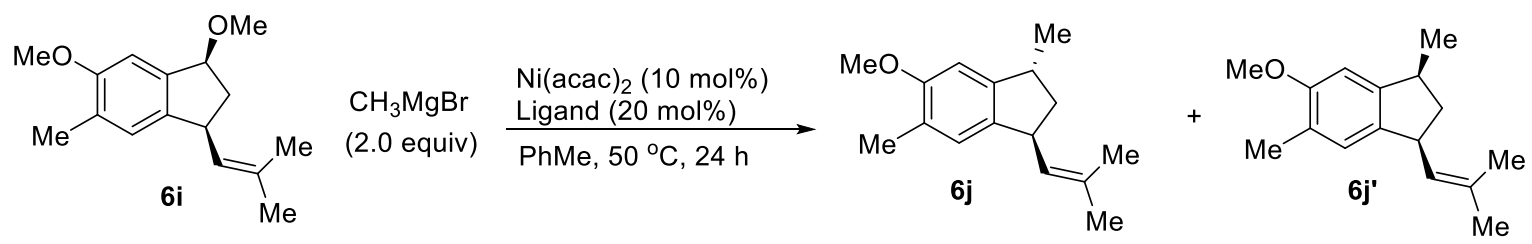

\begin{tabular}{ccccc}
\hline Entry & Ligand & Conv (\%) & GC Yield (\%) & $\operatorname{Dr}(\mathbf{6 j} \mathbf{j} \mathbf{6 j})$ \\
\hline 1 & dppe & 100 & 88 & $1.8: 1$ \\
2 & dppp & 45 & 27 & $1.9: 1$ \\
3 & dppb & 100 & 95 & $2.0: 1$ \\
4 & dppf & 0 & 0 & - \\
5 & Xantphos & 0 & 0 & -
\end{tabular}




\begin{tabular}{ccccc}
6 & DPEPhos & 25 & 15 & $1.8: 1$ \\
7 & rac-BINAP & 0 & 0 & - \\
9 & no ligand & 0 & 0 & - \\
$9^{[\mathrm{a}]}$ & dppb & 100 & 98 & $2.0: 1$ \\
\hline
\end{tabular}

[a] 4 equiv of $\mathrm{MeMgBr}$.

\section{Comparison between nickel-catalyzed reductive cyclization and palladium-catalyzed reductive Heck reaction}

Nickel-catalyzed reaction:

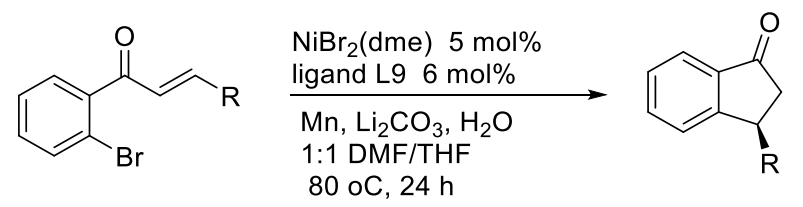

Palladium-catalyzed reaction:

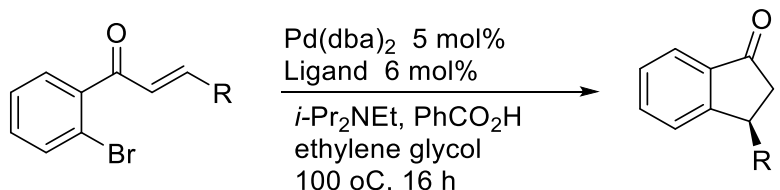

Palladium-catalyzed reductive Heck reaction: In an argon-filled glove box, $\mathrm{Pd}(\mathrm{dba})_{2}(2.8 \mathrm{mg}, 0.005 \mathrm{mmol}, 5$ mol\%), spiro-bisphosphine (0.006 mmol, $6 \mathrm{~mol} \%)$ and degassed ethylene glycol $(0.5 \mathrm{~mL})$ were charged into a 10-mL reaction tube. After stirring for $10 \mathrm{~min}$, benzoic acid (12.0 mg, $0.10 \mathrm{mmol}, 1$ equiv), GC standard $n$ dodecane $(10 \mu \mathrm{L}), N$-diisopropylethylamine $(39.0 \mathrm{mg}, 0.30 \mathrm{mmol}, 3$ equiv) and $(E)$-o-bromochalcone $(0.1$ mmol, 1 equiv) were added. The mixture was vigorously stirred in a preheated oil bath at $100{ }^{\circ} \mathrm{C}$ for $16 \mathrm{~h}$. The reaction mixture was cooled to room temperature, diluted by EtOAc and passed through a short plug of silica gel with diethyl ether washing. The filtrate was used in GC analysis to determine the conversion of starting material and calibrated GC yield of the desired Heck product. Chiral HPLC analysis was performed on the filtrate to determine the enantioselectivity of the product.

Table S8. Comparison between reductive cyclization using nickel/semicorrin $\mathbf{L 9}$ and reductive Heck reaction using palladium catalysts

\begin{tabular}{|c|c|c|c|c|c|c|c|c|c|c|c|c|c|}
\hline \multirow[t]{2}{*}{ Entry } & \multirow[t]{2}{*}{ Products } & \multicolumn{3}{|c|}{$\mathrm{NiBr}_{2}(\mathrm{dme}), \mathbf{L 9}$} & \multicolumn{3}{|c|}{$\begin{array}{l}\text { Pd-catalyzed reaction } \\
\text { condition with } \\
(\boldsymbol{R}) \text {-SDP }\end{array}$} & \multicolumn{3}{|c|}{$\begin{array}{l}\text { Pd-catalyzed reaction } \\
\text { condition with } \\
(\boldsymbol{R}) \text {-Tol-SDP }\end{array}$} & \multicolumn{3}{|c|}{$\begin{array}{l}\text { Pd-catalyzed reaction } \\
\text { condition with } \\
\text { (R)-Xyl-SDP }\end{array}$} \\
\hline & & $\begin{array}{c}\text { Conv. } \\
(\%)\end{array}$ & $\begin{array}{c}\text { Yield } \\
(\%)\end{array}$ & $\begin{array}{l}\mathrm{Ee} \\
(\%)\end{array}$ & $\begin{array}{c}\text { Conv. } \\
(\%)\end{array}$ & $\begin{array}{c}\text { Yield } \\
(\%)\end{array}$ & $\begin{array}{l}\mathrm{Ee} \\
(\%)\end{array}$ & $\begin{array}{c}\text { Conv. } \\
(\%)\end{array}$ & $\begin{array}{c}\text { Yield } \\
(\%)\end{array}$ & $\begin{array}{l}\mathrm{Ee} \\
(\%)\end{array}$ & $\begin{array}{c}\text { Conv. } \\
(\%)\end{array}$ & $\begin{array}{l}\text { Yield } \\
(\%)\end{array}$ & $\begin{array}{l}\mathrm{Ee} \\
(\%)\end{array}$ \\
\hline 1 & & $\begin{array}{l}(100 \\
\text { with } \\
\text { L8) }\end{array}$ & (70) & (81) & 100 & 0 & $\begin{array}{lll}- & -\end{array}$ & 100 & 0 & - & 100 & 0 & - \\
\hline & & & & & & & & & & & & & \\
\hline
\end{tabular}




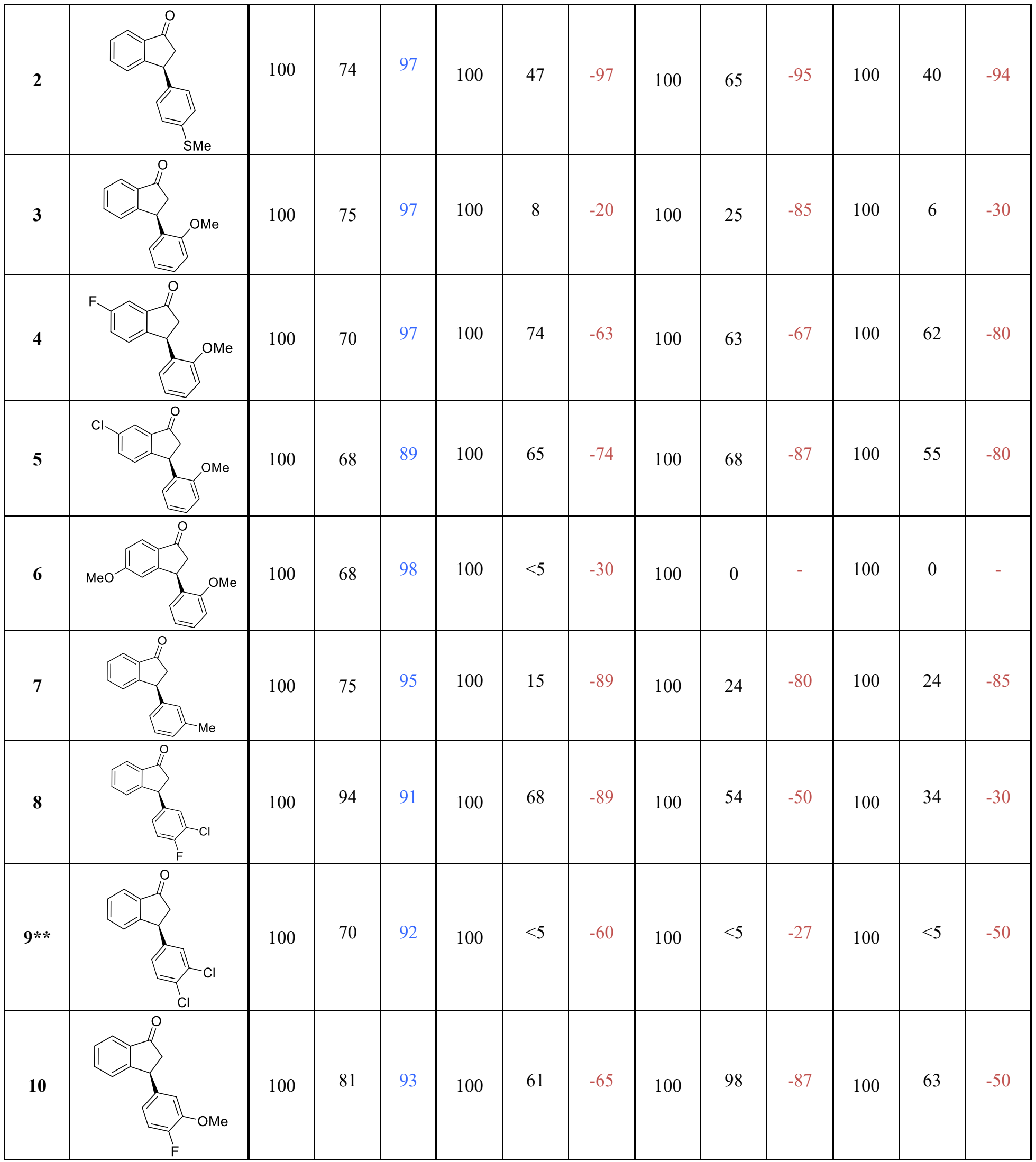




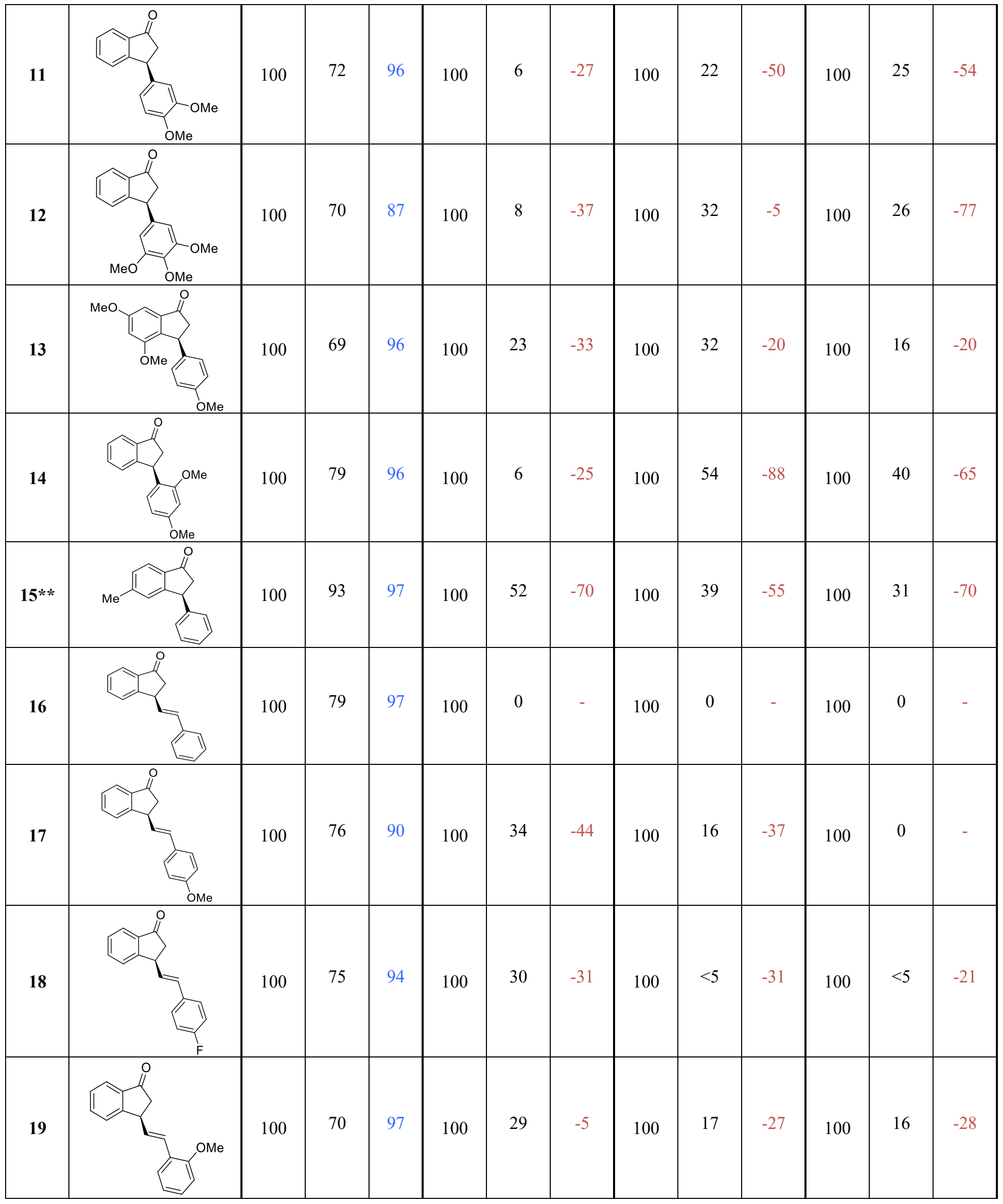




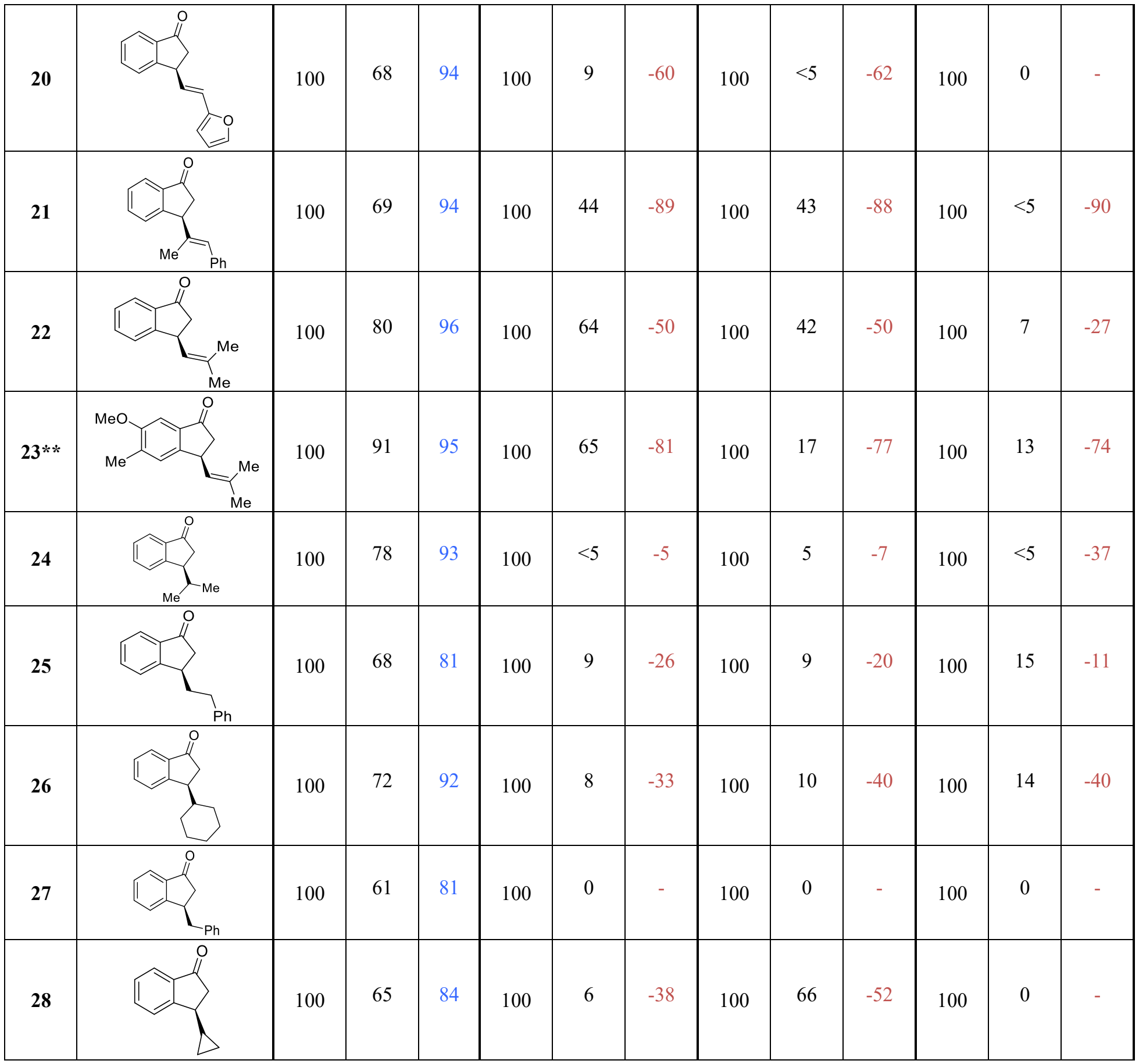

Table S9. Comparison between nickel reductive cyclization using 5 mol\% nickel and 6 mol\% L8 and L9

\begin{tabular}{|c|c|c|c|c|c|c|c|}
\hline \multirow{3}{*}{ Entry } & \multirow{3}{*}{ Products } & \multicolumn{3}{|c|}{$\mathrm{NiBr}_{2}(\mathrm{dme}), \mathbf{4 8}$} & \multicolumn{3}{c|}{$\mathrm{NiBr}_{2}$ (dme), L9 } \\
\cline { 3 - 8 } & & Conv. & GC Yield & Ee & Conv. & GC Yield & Ee \\
& & $(\%)$ & $(\%)$ & $(\%)$ & $(\%)$ & $(\%)$ & $(\%)$ \\
\hline
\end{tabular}




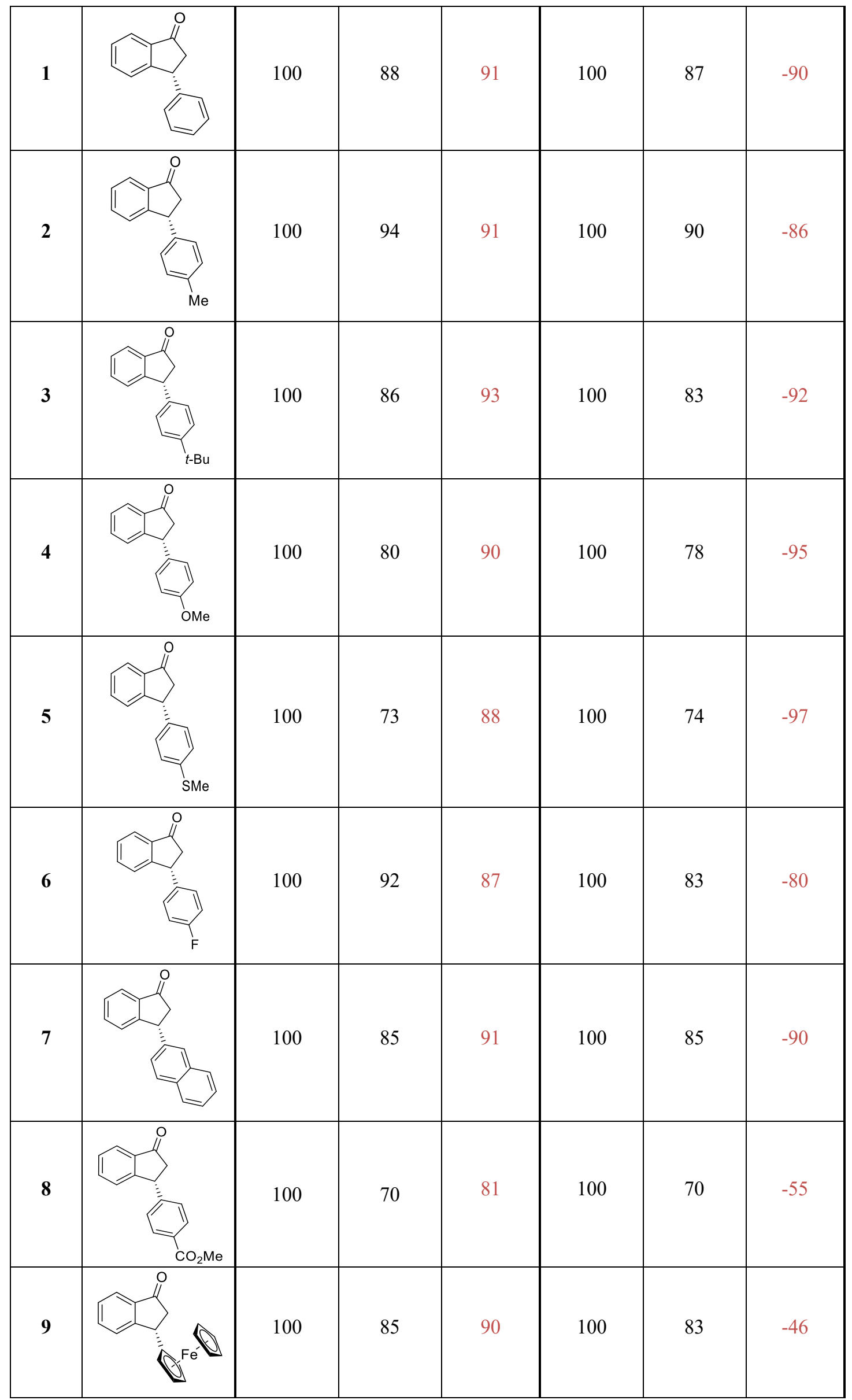




\section{Synthesis of $(E)-2$ '-bromochalcones}

A typical procedure: A solution of $\mathrm{NaOH}(0.3 \mathrm{~g}, 7.5 \mathrm{mmol})$ in water $(2 \mathrm{~mL})$ and ethanol $(20 \mathrm{~mL})$ was cooled to $0{ }^{\circ} \mathrm{C}, 2^{\prime}$-bromoacetophenone ( $5 \mathrm{mmol}, 1.0$ equiv) and aldehyde (5.5 mmol, 1.1 equiv) were slowly added successively. The reaction mixture was allowed to warm to room temperature overnight. After $12 \mathrm{~h}$ the reaction mixture was diluted with EtOAc $(100 \mathrm{~mL} \times 3)$, the organic phase was separated and washed with a saturated solution of $\mathrm{NH}_{4} \mathrm{Cl}$ and dried over $\mathrm{Na}_{2} \mathrm{SO}_{4}$. The solvent was evaporated and the crude material was purified by column chromatography (hexane/ EtOAc 20:1) to yield the desired product. All the new chalcones are fully characterized below.<smiles>CCCc1ccc(/C=C/C(=O)c2ccccc2Br)cc1</smiles>

\section{(E)-2'-Bromo-4-i-propylchalcone (1c)}

${ }^{1} \mathrm{H}$ NMR (400 MHz, $\left.\mathrm{CDCl}_{3}\right): \delta 7.64(\mathrm{~d}, J=7.9 \mathrm{~Hz}, 1 \mathrm{H}), 7.49(\mathrm{~d}, J=8.2 \mathrm{~Hz}, 2 \mathrm{H}), 7.43-7.37$ (m, 3H), 7.35-7.20 $(\mathrm{m}, 1 \mathrm{H}), 7.28-7.24(\mathrm{~m}, 2 \mathrm{H}), 7.05(\mathrm{~d}, J=16.1 \mathrm{~Hz}, 1 \mathrm{H}), 2.93$ (heptet, $J=6.9 \mathrm{~Hz}, 1 \mathrm{H}), 1.25(\mathrm{~d}, J=6.9 \mathrm{~Hz}, 6 \mathrm{H})$. ${ }^{13} \mathrm{C} \mathrm{NMR}\left(101 \mathrm{MHz}, \mathrm{CDCl}_{3}\right): \delta 195.0,152.6,147.0,141.4,133.5,132.2,131.4,129.3,128.9,127.4,127.3$, $125.5,119.6,34.3,23.9$.

MS (ESI ${ }^{+}$: Calcd for $\mathrm{C}_{18} \mathrm{H}_{18} \mathrm{BrO}[\mathrm{M}+\mathrm{H}]^{+}:$329.05. Found: 329.12 .<smiles>CC(=O)c1ccc(/C=C/C(=O)c2ccccc2Br)cc1</smiles>

\section{(E)-2'-Bromo-4-methoxycarbonylchalcone (1i)}

${ }^{1} \mathrm{H}$ NMR (400 MHz, $\left.\mathrm{CDCl}_{3}\right): \delta 8.06(\mathrm{~d}, J=8.3 \mathrm{~Hz}, 2 \mathrm{H}), 7.68-7.60(\mathrm{~m}, 3 \mathrm{H}), 7.49-7.33(\mathrm{~m}, 4 \mathrm{H}), 7.18(\mathrm{~d}, J=16.1$ $\mathrm{Hz}, 1 \mathrm{H}), 3.93$ (s, 3H).

${ }^{13} \mathrm{C} \mathrm{NMR}\left(101 \mathrm{MHz}, \mathrm{CDCl}_{3}\right): \delta 194.3,166.5,144.7,141.0,138.7,133.6,131.9,131.8,130.3,129.4,128.5$, $128.1,127.6,119.6,52.4$.

MS (ESI ${ }^{+}$): Calcd for $\mathrm{C}_{17} \mathrm{H}_{14} \mathrm{BrO}_{3}[\mathrm{M}+\mathrm{H}]^{+}:$345.01. Found: 345.00 . 


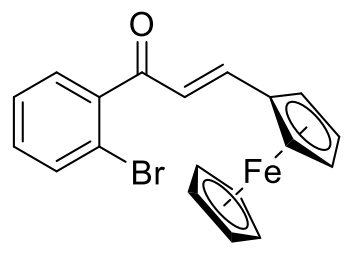

(E)-3-(1-Ferrocenyl)-1-(2'-bromophenyl)-2-propen-1-one (11)

${ }^{1} \mathrm{H}$ NMR (400 MHz, $\left.\mathrm{CDCl}_{3}\right): \delta 7.65(\mathrm{~d}, J=7.8 \mathrm{~Hz}, 1 \mathrm{H}), 7.45-7.20(\mathrm{~m}, 4 \mathrm{H}), 6.67(\mathrm{~d}, J=15.8 \mathrm{~Hz}, 1 \mathrm{H}), 4.54-4.50$ $(\mathrm{m}, 4 \mathrm{H}), 4.20(\mathrm{~s}, 5 \mathrm{H})$.

${ }^{13} \mathrm{C}$ NMR (101 MHz, $\left.\mathrm{CDCl}_{3}\right): \delta$ 194.6, 149.9, 141.9, 133.4, 131.0, 129.0, 127.5, 124.0, 119.4, 78.4, 72.0, 70.0, 69.4 .

MS (ESI $\left.{ }^{+}\right)$: Calcd for $\mathrm{C}_{19} \mathrm{H}_{16} \mathrm{BrFeO}[\mathrm{M}+\mathrm{H}]^{+}:$394.97. Found: 394.99 .<smiles>COc1ccc(/C=C/C(=O)c2ccccc2Br)cc1</smiles>

\section{(E)-2'-Bromo-4-methylthiochalcone (3d)}

${ }^{1} \mathrm{H}$ NMR (400 MHz, $\left.\mathrm{CDCl}_{3}\right): \delta 7.77(\mathrm{~d}, J=16.3 \mathrm{~Hz}, 1 \mathrm{H}), 7.64(\mathrm{~d}, J=7.9 \mathrm{~Hz}, 1 \mathrm{H}), 7.56(\mathrm{~d}, J=7.7 \mathrm{~Hz}, 1 \mathrm{H})$, 7.46-7.28 (m, 4H), $7.20(\mathrm{~d}, J=16.3 \mathrm{~Hz}, 1 \mathrm{H}), 6.96$ ( $\psi \mathrm{t}, J=7.5 \mathrm{~Hz}, 1 \mathrm{H}), 6.92(\mathrm{~d}, J=8.4 \mathrm{~Hz}, 1 \mathrm{H}), 3.86(\mathrm{~s}, 3 \mathrm{H})$. ${ }^{13} \mathrm{C}$ NMR (101 MHz, $\left.\mathrm{CDCl}_{3}\right): \delta 195.4,158.9,142.4,141.6,133.5,132.4,131.3,129.43,129.35,127.4,126.8$, 123.6, 120.9, 119.7, 111.4, 55.7.

MS (ESI ${ }^{+}$): Calcd for $\mathrm{C}_{16} \mathrm{H}_{14} \mathrm{BrOS}[\mathrm{M}+\mathrm{H}]^{+}:$332.99. Found: 332.60 .<smiles>COc1cc(/C=C/C(=O)c2ccccc2Br)ccc1F</smiles>

\section{(E)-2'-Bromo-4-fluoro-3-methoxychalcone (3g)}

${ }^{1} \mathrm{H}$ NMR (400 MHz, $\left.\mathrm{CDCl}_{3}\right): \delta 7.66(\mathrm{~d}, J=7.9 \mathrm{~Hz}, 1 \mathrm{H}), 7.42(\mathrm{~d}, J=4.3 \mathrm{~Hz}, 2 \mathrm{H}), 7.39-7.32(\mathrm{~m}, 2 \mathrm{H}), 7.17-7.06$ (m, 3H), $7.00(\mathrm{~d}, J=16.1 \mathrm{~Hz}, 1 \mathrm{H}), 3.92(\mathrm{~s}, 3 \mathrm{H})$.

${ }^{13} \mathrm{C}$ NMR (101 MHz, $\left.\mathrm{CDCl}_{3}\right): \delta 194.6,154.3\left(\mathrm{~d}, J_{C-F}=252.9 \mathrm{~Hz}\right), 148.3\left(\mathrm{~d}, J_{C-F}=11.3 \mathrm{~Hz}\right), 145.8,141.2$, 133.6, 131.6, $131.2\left(\mathrm{~d}, J_{C-F}=3.9 \mathrm{~Hz}\right), 129.3,127.5,126.1\left(\mathrm{~d}, J_{C-F}=2.4 \mathrm{~Hz}\right), 122.4\left(\mathrm{~d}, J_{C-F}=7.2 \mathrm{~Hz}\right), 119.6$, $116.7\left(\mathrm{~d}, J_{C-F}=19.0 \mathrm{~Hz}\right), 112.8,56.4$.

${ }^{19} \mathrm{~F}$ NMR (377 MHz, $\left.\mathrm{CDCl}_{3}\right): \delta-129.8$.

MS (ESI ${ }^{+}$): Calcd for $\mathrm{C}_{16} \mathrm{H}_{13} \mathrm{BrFO}_{2}[\mathrm{M}+\mathrm{H}]^{+}:$: 335.01. Found: 335.06. 
<smiles>O=C(/C=C/c1ccc(F)c(Cl)c1)c1ccccc1Br</smiles>

\section{(E)-2'-Bromo-3-chloro-4-fluorochalcone (3i)}

${ }^{1} \mathrm{H}$ NMR (400 MHz, $\left.\mathrm{CDCl}_{3}\right): \delta 7.65(\mathrm{~d}, J=7.8 \mathrm{~Hz}, 1 \mathrm{H}), 7.61(\mathrm{dd}, J=7.0,2.2 \mathrm{~Hz}, 1 \mathrm{H}), 7.47-7.39$ (m, 3H), 7.38$7.31(\mathrm{~m}, 2 \mathrm{H}), 7.17(\psi \mathrm{t}, J=8.6 \mathrm{~Hz}, 1 \mathrm{H}), 7.03(\mathrm{~d}, J=16.1 \mathrm{~Hz}, 1 \mathrm{H})$.

${ }^{13} \mathrm{C} \mathrm{NMR}\left(101 \mathrm{MHz}, \mathrm{CDCl}_{3}\right): \delta 194.1,159.5\left(\mathrm{~d}, J_{C-F}=254.7 \mathrm{~Hz}\right.$ ), $143.5\left(\mathrm{~d}, J_{C-F}=1.4 \mathrm{~Hz}\right), 141.0,133.6,132.0$ $\left(\mathrm{d}, J_{C-F}=4.1 \mathrm{~Hz}\right), 131.8,130.7,129.4,128.6\left(\mathrm{~d}, J_{C-F}=7.6 \mathrm{~Hz}\right), 127.6,127.0\left(\mathrm{~d}, J_{C-F}=2.4 \mathrm{~Hz}\right), 122.1\left(\mathrm{~d}, J_{C-F}=\right.$ 18.4 Hz), 119.6, $117.4\left(\mathrm{~d}, J_{C-F}=21.7 \mathrm{~Hz}\right)$.

${ }^{19} \mathrm{~F}$ NMR (377 MHz, $\left.\mathrm{CDCl}_{3}\right): \delta-111.0$.

MS (ESI ${ }^{+}$): Calcd for $\mathrm{C}_{16} \mathrm{H}_{13} \mathrm{BrClFO}[\mathrm{M}+\mathrm{H}]^{+}:$338.96. Found: 339.02.<smiles>O=C(/C=C/c1ccsc1)c1ccccc1Br</smiles>

\section{(E)-1-(2-Bromophenyl)-3-(thiophen-3-yl)prop-2-en-1-one (3k)}

${ }^{1} \mathrm{H}$ NMR (400 MHz, $\left.\mathrm{CDCl}_{3}\right): \delta 7.64(\mathrm{~d}, J=7.9 \mathrm{~Hz}, 1 \mathrm{H}), 7.56-7.53(\mathrm{~m}, 1 \mathrm{H}), 7.41-7.39(\mathrm{~m}, 2 \mathrm{H}), 7.38-7.29(\mathrm{~m}$, $4 \mathrm{H}), 6.90(\mathrm{~d}, J=16.0 \mathrm{~Hz}, 1 \mathrm{H})$.

${ }^{13} \mathrm{C} \mathrm{NMR}\left(101 \mathrm{MHz}, \mathrm{CDCl}_{3}\right): \delta 195.2,141.3,140.2,137.9,133.5,131.4,129.7,129.2,127.5,127.4,126.2$, 125.4, 119.6.

MS (ESI ${ }^{+}$): Calcd for $\mathrm{C}_{13} \mathrm{H}_{10} \mathrm{BrOS}[\mathrm{M}+\mathrm{H}]^{+}:$292.96. Found: 293.07.<smiles>O=C(/C=C/c1ccoc1)c1ccccc1Br</smiles>

\section{(E)-1-(2-Bromophenyl)-3-(furan-3-yl)prop-2-en-1-one (3l)}

${ }^{1} \mathrm{H}$ NMR $\left(400 \mathrm{MHz}, \mathrm{CDCl}_{3}\right): \delta$ 7.68-7.61 (m, 1H), 7.54 (d, J=1.5 Hz, 1H), 7.44-7.37 (m, 2H), 7.34-7.16 (m, 1H), 7.23-7.00 (m, 1H), 6.98 (d, $J=15.8 \mathrm{~Hz}, 1 \mathrm{H}), 6.70(\mathrm{~d}, J=3.4 \mathrm{~Hz}, 1 \mathrm{H}), 6.51$ (dd, $J=3.4,1.8 \mathrm{~Hz}, 1 \mathrm{H})$. ${ }^{13} \mathrm{C}$ NMR $\left(101 \mathrm{MHz}, \mathrm{CDCl}_{3}\right): \delta 194.3,151.2,145.7,141.3,133.6,132.4,131.5,129.3,127.5,123.7,119.7$, $116.9,112.9$.

MS (ESI ${ }^{+}$): Calcd for $\mathrm{C}_{13} \mathrm{H}_{10} \mathrm{BrO}_{2}[\mathrm{M}+\mathrm{H}]^{+}:$: 276.96. Found: 277.11 .<smiles>COc1ccccc1/C=C/C(=O)c1cc(F)ccc1Br</smiles> 
(E)-2'-Bromo-5'-fluoro-2-methoxychalcone (3m)

${ }^{1} \mathrm{H}$ NMR (400 MHz, $\mathrm{CDCl}_{3}$ ): $\delta 7.77$ (d, $\left.J=16.3 \mathrm{~Hz}, 1 \mathrm{H}\right), 7.59-7.55(\mathrm{~m}, 2 \mathrm{H}), 7.42-7.27$ (m, 1H), 7.20-7.12 (m, 2H), 7.09-7.03 (m, 1H), 6.98 ( $\psi \mathrm{t}, J=7.5 \mathrm{~Hz}, 1 \mathrm{H}), 6.92$ (d, $J=8.3 \mathrm{~Hz}, 1 \mathrm{H}), 3.87$ (s, 3H).

${ }^{13} \mathrm{C}$ NMR (101 MHz, $\left.\mathrm{CDCl}_{3}\right): \delta 193.9,161.8\left(\mathrm{~d}, J_{C-F}=249.4 \mathrm{~Hz}\right), 159.0,143.1\left(\mathrm{~d}, J_{C-F}=10.6 \mathrm{~Hz}\right), 135.0(\mathrm{~d}$, $\left.J_{C-F}=7.7 \mathrm{~Hz}\right), 132.6,129.5,126.2,123.3,121.0,120.1,118.6\left(\mathrm{~d}, J_{C-F}=22.5 \mathrm{~Hz}\right), 116.6\left(\mathrm{~d}, J_{C-F}=23.9 \mathrm{~Hz}\right)$, $113.9\left(\mathrm{~d}, J_{C-F}=3.5 \mathrm{~Hz}\right), 111.4,55.7$.

${ }^{19} \mathrm{~F}$ NMR (377 MHz, $\left.\mathrm{CDCl}_{3}\right): \delta-114.0$.

MS (ESI ${ }^{+}$): Calcd for $\mathrm{C}_{16} \mathrm{H}_{13} \mathrm{BrFO}_{2}[\mathrm{M}+\mathrm{H}]^{+}:$335.01. Found: 335.02 .<smiles>COc1ccccc1/C=C/C(=O)c1cc(Cl)ccc1Br</smiles>

\section{(E)-2'-Bromo-5'-chloro-2-methoxychalcone (3n)}

${ }^{1} \mathrm{H}$ NMR (400 MHz, $\left.\mathrm{CDCl}_{3}\right): \delta 7.77(\mathrm{~d}, J=16.3 \mathrm{~Hz}, 1 \mathrm{H}), 7.57-7.55(\mathrm{~m}, 2 \mathrm{H}), 7.43-7.36(\mathrm{~m}, 2 \mathrm{H}), 7.29(\mathrm{dd}, J=$ 8.5, 2.4 Hz, 1H), 7.14 (d, $J=15.1 \mathrm{~Hz}, 1 \mathrm{H}), 6.98$ ( $\psi \mathrm{t}, J=7.5 \mathrm{~Hz}, 1 \mathrm{H}), 6.92$ (d, $J=8.3 \mathrm{~Hz}, 1 \mathrm{H}), 3.87$ (s, 3H). ${ }^{13} \mathrm{C} \mathrm{NMR}\left(101 \mathrm{MHz}, \mathrm{CDCl}_{3}\right): \delta 193.9,159.0,143.1,142.9,134.7,133.8,132.7,131.3,129.6,129.3,126.2$, 123.3, 121.0, 117.6, 111.4, 55.7.

MS (ESI $)$ : Calcd for $\mathrm{C}_{16} \mathrm{H}_{13} \mathrm{BrClO}_{2}[\mathrm{M}+\mathrm{H}]^{+}:$350.98. Found: 351.00 .<smiles>COc1ccc(C(=O)/C=C/c2ccccc2OC)c(Br)c1</smiles>

\section{(E)-2'-Bromo-4'-methoxy-2-methoxychalcone (3o)}

${ }^{1} \mathrm{H}$ NMR (400 MHz, $\left.\mathrm{CDCl}_{3}\right): \delta 7.79(\mathrm{~d}, J=16.2 \mathrm{~Hz}, 1 \mathrm{H}), 7.57-7.49(\mathrm{~m}, 2 \mathrm{H}), 7.39-7.36(\mathrm{~m}, 1 \mathrm{H}), 7.19(\mathrm{~d}, J=$ $16.2 \mathrm{~Hz}, 1 \mathrm{H}), 6.99-6.87(\mathrm{~m}, 4 \mathrm{H}), 3.86(\mathrm{~s}, 3 \mathrm{H}), 3.82(\mathrm{~s}, 3 \mathrm{H})$.

${ }^{13} \mathrm{C} \mathrm{NMR}\left(101 \mathrm{MHz}, \mathrm{CDCl}_{3}\right): \delta 195.3,159.0,142.5,142.4,134.4,132.5,129.6,126.8,123.7,121.1,117.8$, $114.5,111.5,110.1,55.9,55.8$.

MS (ESI $\left.{ }^{+}\right)$: Calcd for $\mathrm{C}_{17} \mathrm{H}_{16} \mathrm{BrO}_{3}[\mathrm{M}+\mathrm{H}]^{+}:$347.03. Found: 346.93 .<smiles>COc1ccc(/C=C/C(=O)c2cc(OC)cc(OC)c2Br)cc1</smiles>

(E)-2'-Bromo-3',5'-dimethoxy-4-methoxychalcone (3q) 
${ }^{1} \mathrm{H}$ NMR (400 MHz, $\left.\mathrm{CDCl}_{3}\right): \delta 7.50(\mathrm{~d}, J=8.3 \mathrm{~Hz}, 2 \mathrm{H}), 7.36(\mathrm{~d}, J=16.1 \mathrm{~Hz}, 1 \mathrm{H}), 6.92-6.89(\mathrm{~m}, 3 \mathrm{H}), 6.56-6.51$ (m, 2H), $3.90(\mathrm{~s}, 1 \mathrm{H}), 3.83(\mathrm{~s}, 1 \mathrm{H}), 3.81(\mathrm{~s}, 1 \mathrm{H})$.

${ }^{13} \mathrm{C}$ NMR (101 MHz, $\left.\mathrm{CDCl}_{3}\right): \delta 195.2,162.1,160.1,156.9,146.9,143.4,130.5,127.2,124.1,114.6,104.6$, $100.9,99.9,56.5,55.8,55.5$.

MS (ESI ${ }^{+}$): Calcd for $\mathrm{C}_{18} \mathrm{H}_{18} \mathrm{BrO}_{4}[\mathrm{M}+\mathrm{H}]^{+}:$377.04. Found: 377.02 .<smiles>COc1ccc(/C=C/C=C/C(=O)c2ccccc2Br)cc1</smiles>

\section{(2E,4E)-1-(2-Bromophenyl)-5-(4-methoxyphenyl)penta-2,4-dien-1-one (5b)}

${ }^{1} \mathrm{H}$ NMR (400 MHz, $\left.\mathrm{CDCl}_{3}\right): \delta 7.62(\mathrm{~d}, J=8.0 \mathrm{~Hz}, 1 \mathrm{H}), 7.42-7.36(\mathrm{~m}, 4 \mathrm{H}), 7.33-7.28(\mathrm{~m}, 1 \mathrm{H}), 7.19-7.13(\mathrm{~m}$, $1 \mathrm{H}), 6.90-6.85(\mathrm{~m}, 4 \mathrm{H}), 6.56(\mathrm{~d}, J=15.3 \mathrm{~Hz}, 1 \mathrm{H}), 3.82(\mathrm{~s}, 3 \mathrm{H})$.

${ }^{13} \mathrm{C} \mathrm{NMR}\left(101 \mathrm{MHz}, \mathrm{CDCl}_{3}\right): \delta 195.0,160.9,147.7,142.5,141.5,133.4,131.2,129.1,128.8,128.5,127.4$, $124.7,119.5,114.5,55.5$.

MS (ESI $\left.{ }^{+}\right)$: Calcd for $\mathrm{C}_{18} \mathrm{H}_{16} \mathrm{BrO}_{2}[\mathrm{M}+\mathrm{H}]^{+}:$343.03. Found: 343.05 .<smiles>O=C(/C=C/C=C/c1ccc(F)cc1)c1ccccc1Br</smiles>

\section{(2E,4E)-1-(2-Bromophenyl)-5-(4-fluorophenyl)penta-2,4-dien-1-one (5c)}

${ }^{1} \mathrm{H}$ NMR (400 MHz, $\left.\mathrm{CDCl}_{3}\right): \delta 7.62(\mathrm{~d}, J=8.0 \mathrm{~Hz}, 1 \mathrm{H}), 7.46-7.41(\mathrm{~m}, 2 \mathrm{H}), 7.39-7.36(\mathrm{~m}, 2 \mathrm{H}), 7.33-7.29$ (m, 1H), 7.19-7.13 (m, 1H), $7.04(\psi \mathrm{t}, J=8.6 \mathrm{~Hz}, 2 \mathrm{H}), 6.90-6.86(\mathrm{~m}, 2 \mathrm{H}), 6.62(\mathrm{~d}, J=15.3 \mathrm{~Hz}, 1 \mathrm{H})$.

${ }^{13} \mathrm{C}$ NMR $\left(101 \mathrm{MHz}, \mathrm{CDCl}_{3}\right): \delta 194.8,163.4\left(\mathrm{~d}, J_{C-F}=250.6 \mathrm{~Hz}\right), 146.6,141.3,141.2,133.5,132.2\left(\mathrm{~d}, J_{C-F}=\right.$ $3.4 \mathrm{~Hz}), 131.3,129.5,129.2\left(\mathrm{~d}, J_{C-F}=8.5 \mathrm{~Hz}\right), 129.1,127.4,126.5\left(\mathrm{~d}, J_{C-F}=2.4 \mathrm{~Hz}\right), 119.5,116.1\left(\mathrm{~d}, J_{C-F}=\right.$ $21.9 \mathrm{~Hz})$.

${ }^{19} \mathrm{~F} \mathrm{NMR}\left(377 \mathrm{MHz}, \mathrm{CDCl}_{3}\right): \delta-110.6$.

MS (ESI ${ }^{+}$: Calcd for $\mathrm{C}_{17} \mathrm{H}_{13} \mathrm{BrFO}[\mathrm{M}+\mathrm{H}]^{+}$: 331.01. Found: 331.05 .<smiles>COc1ccccc1/C=C/C=C/C(=O)c1ccccc1Br</smiles>

(2E,4E)-1-(2-Bromophenyl)-5-(2-methoxyphenyl)penta-2,4-dien-1-one (5d) 
${ }^{1} \mathrm{H}$ NMR (400 MHz, $\left.\mathrm{CDCl}_{3}\right): \delta$ 7.65-7.61 (m, 1H), $7.52(\mathrm{dd}, J=7.7,1.5 \mathrm{~Hz}, 1 \mathrm{H}), 7.42-7.34(\mathrm{~m}, 2 \mathrm{H}), 7.34-7.28$ $(\mathrm{m}, 2 \mathrm{H}), 7.26-7.25(\mathrm{~m}, 1 \mathrm{H}), 7.17(\mathrm{dd}, J=15.2,11.2 \mathrm{~Hz}, 1 \mathrm{H}), 7.03(\mathrm{dd}, J=15.5,11.1 \mathrm{~Hz}, 1 \mathrm{H}), 6.95(\psi \mathrm{t}, J=7.3$ $\mathrm{Hz}, 1 \mathrm{H}), 6.89$ (d, $J=8.3 \mathrm{~Hz}, 1 \mathrm{H}), 6.58(\mathrm{~d}, J=15.2 \mathrm{~Hz}, 1 \mathrm{H}), 3.86$ (s, 3H).

${ }^{13} \mathrm{C} \mathrm{NMR}\left(101 \mathrm{MHz}, \mathrm{CDCl}_{3}\right): \delta 195.3,157.8,148.5,141.5,137.9,133.5,131.2,130.8,129.1,127.7,127.4$, $127.3,125.0,121.0,120.1,119.6,111.3,55.7$.

MS (ESI $\left.{ }^{+}\right)$: Calcd for $\mathrm{C}_{18} \mathrm{H}_{16} \mathrm{BrO}_{2}[\mathrm{M}+\mathrm{H}]^{+}:$343.03. Found: 343.04 .<smiles>O=C(/C=C/C=C/c1ccco1)c1ccccc1Br</smiles>

\section{(2E,4E)-1-(2-Bromophenyl)-5-(furan-2-yl)penta-2,4-dien-1-one (5e)}

${ }^{1} \mathrm{H}$ NMR $\left(400 \mathrm{MHz}, \mathrm{CDCl}_{3}\right): \delta 7.62(\mathrm{~d}, J=7.8 \mathrm{~Hz}, 1 \mathrm{H}), 7.46-7.45(\mathrm{~m}, 1 \mathrm{H}), 7.41-7.35(\mathrm{~m}, 2 \mathrm{H}), 7.33-7.28(\mathrm{~m}$, $1 \mathrm{H}), 7.13(\mathrm{dd}, J=15.2,11.3 \mathrm{~Hz}, 1 \mathrm{H}), 6.87(\mathrm{dd}, J=15.3,11.3 \mathrm{~Hz}, 1 \mathrm{H}), 6.70(\mathrm{~d}, J=15.3 \mathrm{~Hz}, 1 \mathrm{H}), 6.61(\mathrm{~d}, J=$ $15.2 \mathrm{~Hz}, 1 \mathrm{H}), 6.48$ (d, $J=3.4 \mathrm{~Hz}, 1 \mathrm{H}), 6.45-6.44(\mathrm{~m}, 1 \mathrm{H})$.

${ }^{13} \mathrm{C} \mathrm{NMR}\left(101 \mathrm{MHz}, \mathrm{CDCl}_{3}\right): \delta 194.7,152.3,146.4,144.3,141.4,133.5,131.3,129.3,129.2,128.8,127.4$, $125.1,119.6,112.9,112.5$.

MS (ESI ${ }^{+}$): Calcd for $\mathrm{C}_{15} \mathrm{H}_{12} \mathrm{BrO}_{2}[\mathrm{M}+\mathrm{H}]^{+}:$303.00. Found: 303.07 .

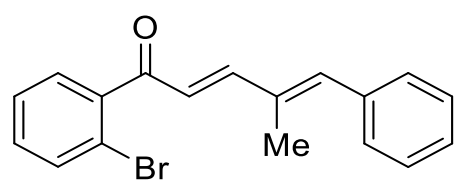

\section{(2E,4E)-1-(2-Bromophenyl)-4-methyl-5-phenylpenta-2,4-dien-1-one (5f)}

${ }^{1} \mathrm{H}$ NMR (400 MHz, $\left.\mathrm{CDCl}_{3}\right): \delta 7.64(\mathrm{~d}, J=7.9 \mathrm{~Hz}, 1 \mathrm{H}), 7.42-7.24(\mathrm{~m}, 9 \mathrm{H}), 6.85(\mathrm{~s}, 1 \mathrm{H}), 6.63(\mathrm{~d}, J=15.7 \mathrm{~Hz}$, $1 \mathrm{H}), 2.12(\mathrm{~s}, 3 \mathrm{H})$.

${ }^{13} \mathrm{C} \mathrm{NMR}\left(101 \mathrm{MHz}, \mathrm{CDCl}_{3}\right): \delta 195.2,152.3,141.6,141.2,136.6,134.7,133.5,131.3,129.7,129.2,128.5$, 128.2, 127.4, 126.0, 119.5, 14.0.

MS (ESI ${ }^{+}$: Calcd for $\mathrm{C}_{18} \mathrm{H}_{16} \mathrm{BrO}[\mathrm{M}+\mathrm{H}]^{+}:$327.04. Found: 327.04 .<smiles>CC(C)=CC=CC(=O)c1ccccc1Br</smiles>

\section{(E)-1-(2-Bromophenyl)-5-methylhexa-2,4-dien-1-one (5g)}

${ }^{1} \mathrm{H}$ NMR (400 MHz, $\left.\mathrm{CDCl}_{3}\right): \delta 7.62(\mathrm{~d}, J=8.2 \mathrm{~Hz}, 1 \mathrm{H}), 7.41-7.34(\mathrm{~m}, 3 \mathrm{H}), 7.31-7.25(\mathrm{~m}, 1 \mathrm{H}), 6.42(\mathrm{~d}, J=15.2$ $\mathrm{Hz}, 1 \mathrm{H}), 6.11(\mathrm{~d}, J=11.6 \mathrm{~Hz}, 1 \mathrm{H}), 1.90(\mathrm{~s}, 3 \mathrm{H}), 1.84(\mathrm{~s}, 3 \mathrm{H})$. 
${ }^{13} \mathrm{C}$ NMR (101 MHz, $\left.\mathrm{CDCl}_{3}\right): \delta 195.1,149.7,143.1,141.7,133.4,131.1,129.1,127.3,127.1,124.6,119.4$, 26.9, 19.3.

MS (ESI ${ }^{+}$: Calcd for $\mathrm{C}_{13} \mathrm{H}_{14} \mathrm{BrO}[\mathrm{M}+\mathrm{H}]^{+}:$265.02. Found: 265.04 .<smiles>O=C(/C=C/C1CC1)c1ccccc1Br</smiles>

\section{(E)-1-(2-Bromophenyl)-3-prop-2-en-1-one (7e)}

${ }^{1} \mathrm{H}$ NMR (400 MHz, $\left.\mathrm{CDCl}_{3}\right): \delta 7.59$ (d, $\left.J=7.9 \mathrm{~Hz}, 1 \mathrm{H}\right), 7.38-7.25(\mathrm{~m}, 3 \mathrm{H}), 6.54(\mathrm{~d}, J=15.6 \mathrm{~Hz}, 1 \mathrm{H}), 6.17(\mathrm{dd}$, $J=15.5,10.0 \mathrm{~Hz}, 1 \mathrm{H}), 1.77-1.57(\mathrm{~m}, 1 \mathrm{H}), 1.10-0.97(\mathrm{~m}, 2 \mathrm{H}), 0.72-0.58(\mathrm{~m}, 2 \mathrm{H})$.

${ }^{13} \mathrm{C}$ NMR (101 MHz, $\left.\mathrm{CDCl}_{3}\right): \delta 194.4,158.2,141.4,133.4,131.1,129.1,127.4,127.3,119.5,15.5,9.7$. MS (ESI ${ }^{+}$: Calcd for $\mathrm{C}_{12} \mathrm{H}_{12} \mathrm{BrO}[\mathrm{M}+\mathrm{H}]^{+}: 251.01$. Found: 251.05 .

\section{Reference}

(1) Yang, J.; Yoshikai, N. J. Am. Chem. Soc. 2014, 136, 16748.

(2) Minatti, A.; Zheng, X.; Buchwald, S. L. J. Org. Chem. 2007, 72, 9253.

(3) Yu, Y. N.; Xu, M. H. J. Org. Chem. 2013, 78, 2736.

(4) Yue, G.; Lei, K.; Hirao, H.; Zhou, J. S. Angew. Chem., Int. Ed. 2015, 54, 6531.

(5) Tang, M. L.; Peng, P.; Liu, Z. Y.; Zhang, J.; Yu, J. M.; Sun, X. Chem. Eur. J. 2016, 22, 14535.

(6) Roesner, S.; Casatejada, J. M.; Elford, T. G.; Sonawane, R. P.; Aggarwal, V. K. Org. Lett. 2007, $13,5740$.

(7) Bianco, G. G.; M. C. Ferraz, H. M. C.; Costa, A. M.; Costa-Lotufo, L. V.; Pessoa, C.; Moraes, M. O.;

Schrems, M. G.; Pfaltz, A.; Silva, L. F. J. Org. Chem. 2009, 2009, 2561. 